Simon Homes, Matthias Heinen, Jadran Vrabec, Johann Fischer

\title{
Evaporation driven by conductive heat transport
}

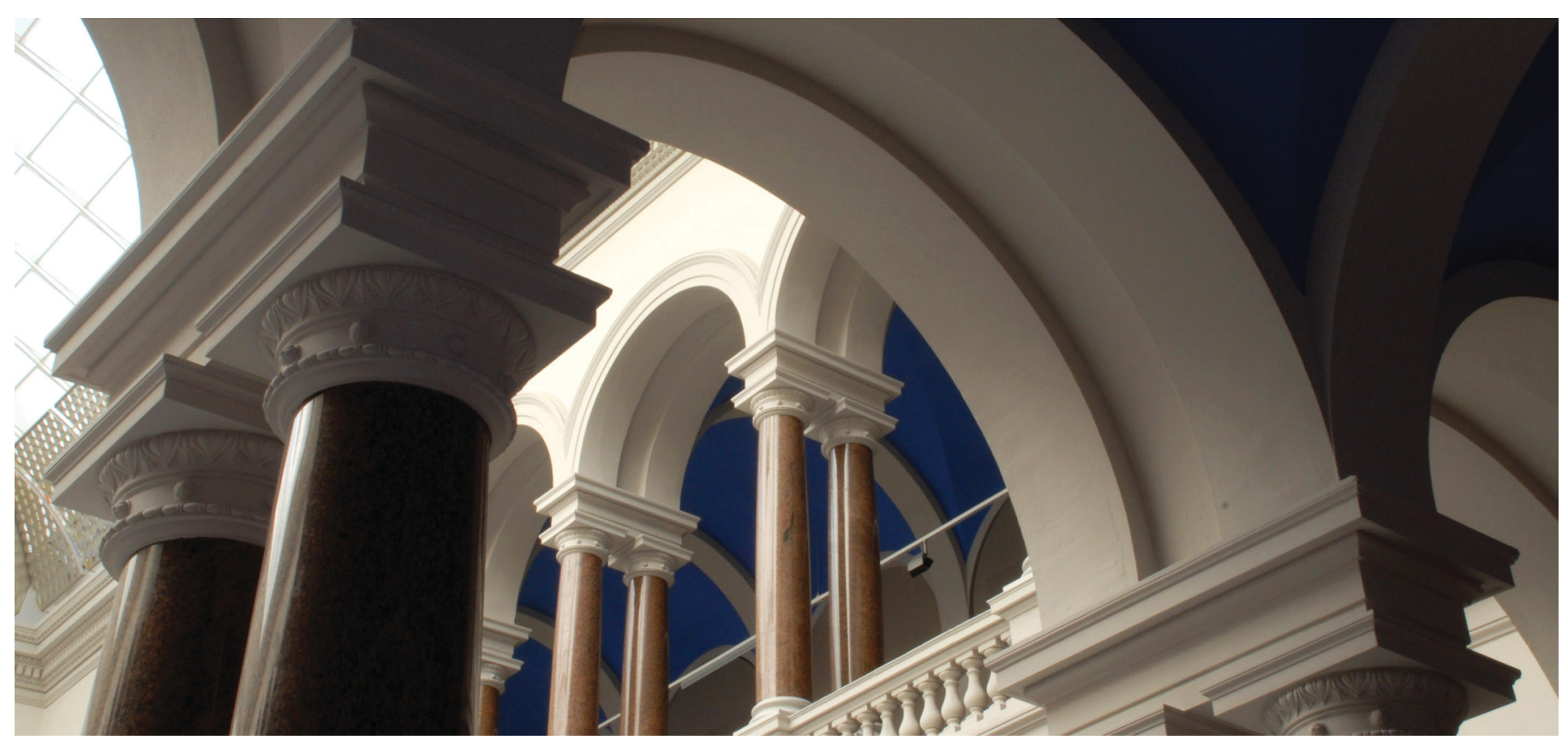

This is an Accepted Manuscript of an article published by Taylor \& Francis in Molecular Physics on 24 Oct 2020, available online: http://www.tandfonline.com/10.1080/00268976.2020.1836410

Homes, S., Heinen, M., Vrabec, J., \& Fischer, J. (2020). Evaporation driven by conductive heat transport. Molecular Physics, e1836410. https://doi.org/10.1080/00268976.2020.1836410 


\title{
Evaporation driven by conductive heat transport
}

\author{
Simon Homes ${ }^{\mathrm{a}}$, Matthias Heinen ${ }^{\mathrm{a}}$, Jadran Vrabec $^{\mathrm{a}, *}$ and Johann Fischer ${ }^{\mathrm{b}}$ \\ aTechnische Universität Berlin, Thermodynamik und Thermische Verfahrenstechnik, \\ Ernst-Reuter-Platz 1, 10587 Berlin, Germany. \\ ${ }^{\mathrm{b}}$ Universität für Bodenkultur, Institut für Verfahrens- und Energietechnik, Muthgasse 107, \\ 1190 Wien, Austria.
}

\begin{abstract}
ARTICLE HISTORY
Compiled October 7, 2020

ABSTRACT

Molecular dynamics simulations are conducted to investigate the evaporation of the truncated $(2.5 \sigma)$ and shifted Lennard-Jones fluid into vacuum. Evaporation is maintained under stationary conditions, while the bulk liquid temperature and the thermal driving force gradient are varied over wide ranges. It is found that the particle flux and the energy flux solely depend on the interface temperature. Both of these quantities are correlated to estimate their values for macroscopically large systems. The latter is analyzed by a hydrodynamic energy balance, considering conductive heat transport by Fourier's law. Following the Hertz-Knudsen approach, the evaporation coefficient is determined and found to be in good agreement with literature data based on the kinetic equation for fluids and molecular dynamics.
\end{abstract}

\section{KEYWORDS}

Evaporation; Evaporation coefficient; Heat transport; Molecular dynamics;

Lennard-Jones fluid;

\section{Introduction}

In 1882, Hertz [1] studied evaporation of liquid mercury into vacuum experimentally and discussed the heat transport from the bulk liquid to the interface, which is driven by a temperature gradient. He suggested an equation for the evaporation flux based on the kinetic theory of gases, which is known as Hertz model or Hertz-Knudsen equation, and relies on the bulk liquid temperature $T_{\text {liq }}$ and the corresponding saturated vapor density $\rho^{\prime \prime}$ as input parameters. About thirty years later, Knudsen [2] also made evaporation experiments with mercury and introduced the evaporation coefficient $\alpha$ as a ratio of the measured evaporation flux and the flux calculated from the Hertz model. These measurements were made at a temperature of $T=293 \mathrm{~K}$ and resulted in an evaporation coefficient $\alpha=0.96$. It should be noted that the critical temperature of mercury is $T_{\mathrm{c}}=1763 \mathrm{~K}[3]$ so that Knudsen made his experiments at a very low reduced temperature of $T / T_{\mathrm{c}}=0.166$.

In the subsequent century, a large number of experimental [4-18], theoretical [19$25]$ and molecular simulation papers [17, 18, 25-47] as well as review articles [48-51], books [52-59] and data compilations [60-63] appeared, of which only some are cited 
here. Despite these efforts, it still seems that theory and simulation often do not match with the experimental findings. Regarding the experiments, we believe that the measured temperatures might be a problem in some cases: What had really been measured and to which position in the vapor-liquid interface region has the measured quantity be allocated to? In our opinion, the physical quantities that can most reliably be measured experimentally are the temperature of the bulk liquid and the particle flux due to evaporation. On the other hand, theories and simulations often neglect the temperature decrease from the bulk liquid to the interface, which is necessary to transport the heat to sustain evaporation.

In our earlier molecular dynamics (MD) simulations on evaporation from a planar liquid interface into vacuum $[26,40]$, the distance between the thermostated liquid region and the interface plane defined by the inflection point (IP) of the density profile was only about $6 \sigma$, with $\sigma$ being the particle diameter. The importance of the length of this non-thermostated liquid region $L_{\mathrm{n}}$ became clear from the article of Frezzotti et al. [20] who had solved a kinetic equation for fluids (KEF) [64, 65]. Fig. 4 in Ref. [20] shows a linearly decreasing temperature profile in the non-thermostated region ranging from the bulk liquid to the density IP over a distance of about $16 \sigma$. Closer inspection of the article of Anisimov et al. [28] showed a similar linear temperature decrease in the non-thermostated region over about $10 \sigma$.

Refs. [20, 28] motivated us to study the region between the thermostated liquid and the interface in more detail with MD [41]. In that communication, we investigated a Lennard-Jones type fluid with a potential truncated and shifted at $2.5 \sigma$, shortly called LJTS2.5, with the critical temperature $T_{\mathrm{c}}=1.08$ in the usual units [66]. For convenience, in the following all quantities are given in these units reduced by the size parameter $\sigma$, the energy parameter $\varepsilon$ and the particle mass $m$, e.g. the particle flux reads $j_{\mathrm{p}} \sigma^{3} \sqrt{m / \varepsilon}$. In Ref. [41], we considered evaporation for the bulk liquid temperature $T_{\text {liq }}=0.8\left(T_{\text {liq }} / T_{\mathrm{c}}=0.74\right)$ and varied $L_{\mathrm{n}}$ from 5.2 to 208 . It emerged that the interface temperature at the IP of the density profile $T_{\text {IP }}$ (symbolized by $T_{\mathrm{i}}$ in Ref. [41]) drops by $17 \%$ and the evaporation flux decreases by a factor of 4.4. Moreover, it was found that the interface temperature $T_{\mathrm{IP}}$ as a function of $L_{\mathrm{n}}$ exhibits an exponential asymptotic behavior from which an extrapolation can be made to estimate $T_{\mathrm{IP}}^{\infty}$ for an infinitely large length of the non-thermostated liquid region $L_{\mathrm{n}} \rightarrow \infty$. It is interesting to note that this extrapolated interface temperature $T_{\mathrm{IP}}^{\infty}$ differs from $T_{\mathrm{IP}}$ at $L_{\mathrm{n}}=208$ by less than $1 \%$, which also allowed for an estimation of the particle flux $j_{\mathrm{p}}^{\infty}$ for $L_{\mathrm{n}} \rightarrow \infty$. It can be supposed that this extrapolation to $L_{\mathrm{n}} \rightarrow \infty$ on the molecular scale is required for a comparison with experimental data on the macroscopic scale. Finally, we compared $j_{\mathrm{p}}^{\infty}$ with the Hertz flux calculated on the basis of the bulk liquid temperature and thus obtained an evaporation coefficient of $\alpha=0.14$.

This evaporation coefficient $\alpha=0.14$ [41] for $T_{\text {liq }} / T_{\mathrm{c}}=0.74$ is much lower than the evaporation coefficient $\alpha=0.51$ given by Frezzotti in Fig. 10 of Ref. [20] for nearly the same reduced bulk liquid temperature $T_{\text {liq }} / T_{\mathrm{c}}=0.73$ that was obtained for $L_{\mathrm{n}} \approx 16$ with KEF. However, Ref. [41] contains also results for $T_{\text {liq }} / T_{\mathrm{c}}=0.74$ and $L_{\mathrm{n}}=15.6$, being $j_{\mathrm{p}}^{\text {sim }}=3.443 \cdot 10^{-3}$ (Table I) and $j^{\mathrm{H}}\left(T_{\text {liq }}\right)=7.058 \cdot 10^{-3}$ for the Hertz flux, which yields $\alpha=0.49$. Hence, the results from both methods are in good mutual agreement under similar boundary conditions.

The temperature dependence of the evaporation coefficient $\alpha$ for $L_{\mathrm{n}} \rightarrow \infty$ remains to be of great interest. Several studies based on MD simulation [28, 32, 33, 40] and KEF [20] found that $\alpha$ rises with decreasing temperature. The temperature interval covered in these articles ranged from $T_{\text {liq }} / T_{\mathrm{c}}=0.534$ [40] to 0.90 [32]. The temperature 
interval that we can consider by simulation with a long non-thermostated liquid region is, however, limited. With reported temperatures between $T_{\text {tr }}=0.55$ and 0.62 [67-69], the triple point poses a lower limit, but fluid simulations were possible down to $T_{\text {liq }}=$ 0.625 without observing crystallization phenomena. The upper limit of the bulk liquid temperature is given by stability issues of the liquid film, since increasing temperature results in declining density of the liquid that is associated with less cohesion. Exposed to a vacuum nearby, such liquid phases are prone to disintegrate. For this reason, $T_{\text {liq }}=0.80$ was selected here as the highest temperature.

In the present work, we performed simulations for $T_{\text {liq }}=0.625,0.65,0.70,0.75$ and 0.80 . Hence, a reduced temperature range $T_{\text {liq }} / T_{\mathrm{c}}$ from 0.58 to 0.74 was covered, which is comparable to the range of $T_{\text {liq }} / T_{\mathrm{c}}=0.595$ to 0.71 considered in Ref. [20]. This allows for an assessment of the consistency between KEF and MD data for $L_{\mathrm{n}} \approx 16$ and to show the effect of increasing $L_{\mathrm{n}}$ up to 208 and extrapolating to $L_{\mathrm{n}} \rightarrow \infty$.

A second topic is the question whether the heat transport through the liquid phase, driving the evaporation process, can be described by equations of hydrodynamics, wherein the heat flux is expressed by Fourier's law. The importance of such considerations was revealed before, e.g. in the work of Hołyst et al. [35] for the evaporation of nanodroplets. Moreover, in Ref. [36], a study on evaporation into vacuum, an interesting discussion on the momentum flux of the vapor flow can be found. Therefore, this question was investigated by a comprehensive analysis of the energy balance, also clarifying the role of the interface temperature $T_{\mathrm{IP}}$ that governs evaporation.

Section 2 presents the simulation method including results of tests for its consistency and accuracy. This is necessary because we employed a simulation method introduced in Ref. [43] to achieve stationary evaporation conditions. This method, however, is different from the one used in Refs. [26, 40] and another method utilized in Ref. [41]. Section 3 deals with particle fluxes, distinguishing between net, forward and backscattered flux, as well as the evaporation coefficient $\alpha$. Moreover, the temperature at the interface is analyzed and a correlation for the particles flux is derived. This section also includes a comparison with experimental evaporation coefficient data. Section 4 presents results for the heat flux as a part of a detailed analysis of the energy balance. Finally, conclusions are drawn.

\section{Simulation method and accuracy}

MD simulations were conducted with the software ls1 mardyn [70]. The particle interactions were modeled by a Lennard-Jones potential that was truncated and shifted at the cutoff radius $r_{\mathrm{c}}=2.5 \sigma[66,71]$

$$
u_{\mathrm{LJTS} 2.5}\left(r_{i j}\right)= \begin{cases}u_{\mathrm{LJ}}\left(r_{i j}\right)-u_{\mathrm{LJ}}(2.5 \sigma), & r_{i j} \leq 2.5 \sigma \\ 0, & r_{i j}>2.5 \sigma\end{cases}
$$

and

$$
u_{\mathrm{LJ}}\left(r_{i j}\right)=4 \varepsilon\left[\left(\frac{\sigma}{r_{i j}}\right)^{12}-\left(\frac{\sigma}{r_{i j}}\right)^{6}\right],
$$


with $u_{\text {LJTS2.5 }}$ being the potential used in this work, $r_{i j}$ the distance between particles $i$ and $j$ and $u_{\mathrm{LJ}}$ the Lennard-Jones potential, which contains the size parameter $\sigma$ and the energy parameter $\varepsilon$. Since that potential is truncated, no long-range corrections had to be applied.

The simulation volume was a rectangular parallelepiped with edge lengths $L_{x}=$ $L_{y}=200$ and $L_{z}$ varying between 470.2 and 673 . It contained one liquid and one vapor phase. The $z$ axis was normal to the planar vapor-liquid interface, with $z=0$ being the position of the interface. There are several approaches to determine the interface position, e.g. the extremum of the interaction forces $(\mathrm{FE})$ or the IP of the density profile, cf. Fig. 1. For the sake of comparability with the results of previous work [41], the interface position was identified by the IP of the density profile. Note that the interface temperature $T_{i}$ in Ref. [41] is not - as stated erroneously there - the temperature at the force extremum $\mathrm{FE}$, but the temperature $T_{\mathrm{IP}}$ at the density IP. Moreover, checks showed that there are no significant changes of the results by using either IP or FE as interface position. Therefore, we assume that other choices of the interface position like the separation of the temperature profiles [41] or the Kapitza position [72] will also show no significant changes.

The simulation volume can be divided into four regions as depicted in Fig. 1, which are briefly described with ascending $z$ : The region in which the liquid was slab-wise thermostated by velocity scaling had a length of $L_{\mathrm{t}}=25$. Together with the nonthermostated liquid region, which spans in $z$ direction over $L_{n}$, it constituted the liquid phase. The vapor phase had a length of $L_{\mathrm{vap}}=400$ and ended with the onset of the vacuum region $L_{\mathrm{vac}}$.

The density of each phase was initially set to the respective saturated density, corresponding to the temperature of the bulk liquid [66]. Consequently, the total number of particles varied from about 1.2 to $8.4 \cdot 10^{6}$, depending on the temperature and the length of the non-thermostated liquid region. Each phase was equilibrated separately for $5 \cdot 10^{5}$ time steps $\Delta t=0.001824$. After joining the two phases, a second equilibration in the vapor-liquid equilibrium (VLE) state was conducted for another $5 \cdot 10^{5}$ time steps. Subsequently, the production run was carried out with the following boundary conditions: The boundaries parallel to the $x z$ and $y z$ plane were set to be periodic, the vacuum was enabled in the according domain and particles reaching this subvolume were deleted. In order to maintain a stationary evaporation process, the liquid phase had to be replenished with new particles. This was achieved with the method suggested in Ref. [43]. A slab of liquid was continually slid into the simulation volume over the according spatial boundary. The velocity of the slab was controlled such that the number of particles removed from and replenished into the simulation volume was balanced. This approach differs substantially from insertion methods, e.g. the one proposed in Ref. [73], based on search algorithms for appropriate insertion positions of low potential energy. Because of its characteristic and the fact that the position of integrating new particles into the liquid phase is far away from the regions of interest, the influence of the integration process on, e.g. the local internal energy, is not important.

Quantities of interest, like the temperature, density, force and flux profiles, were averaged over $2.5 \cdot 10^{4}$ time steps. Spatially, the profiles were sampled in bins of width 0.25 along the coordinate $z$.

Present simulations were conducted until a stationary state was reached and maintained for a sufficient sampling time. The number of time steps, which were needed until stationarity, depends heavily on the extent of the non-thermostated liquid region. For $L_{\mathrm{n}} \leq 52$, stationarity was achieved after less than $3.5 \cdot 10^{6}$ time steps. For 

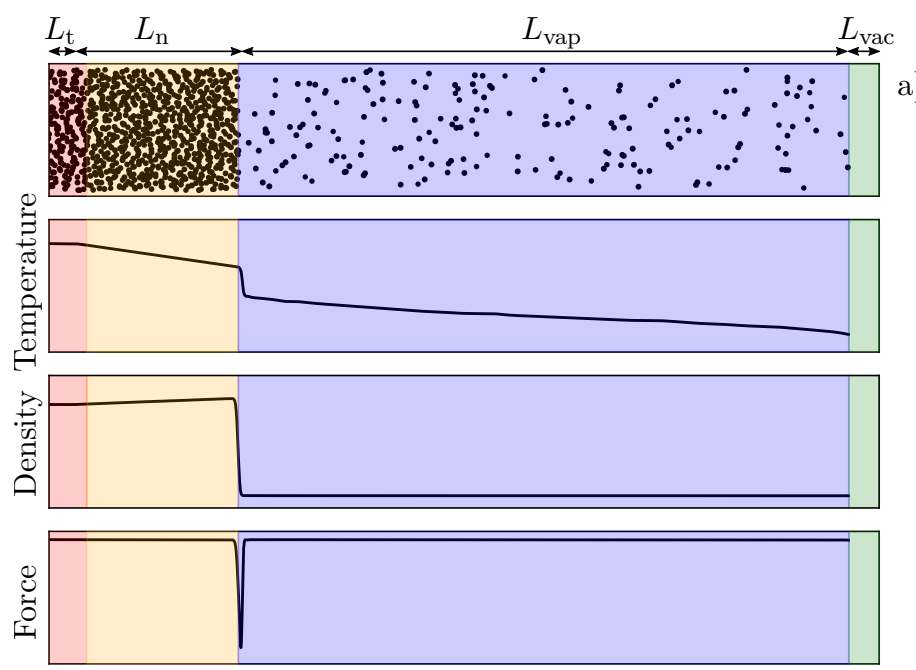

Coordinate $z$
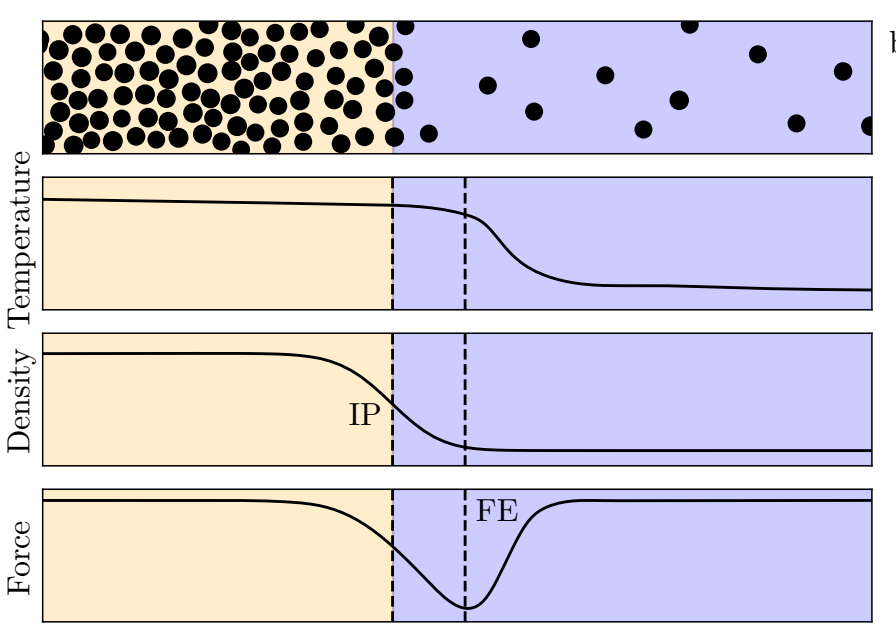

Coordinate $z$

Figure 1. Cut through the simulation volume with profiles of temperature, density and force over the spatial coordinate $z$. Background colors mark different fluid regions: red - thermostated liquid; yellow - nonthermostated liquid; blue - vapor; green - vacuum. a) Overview of the entire simulation volume; b) Detailed view on the interface region. 

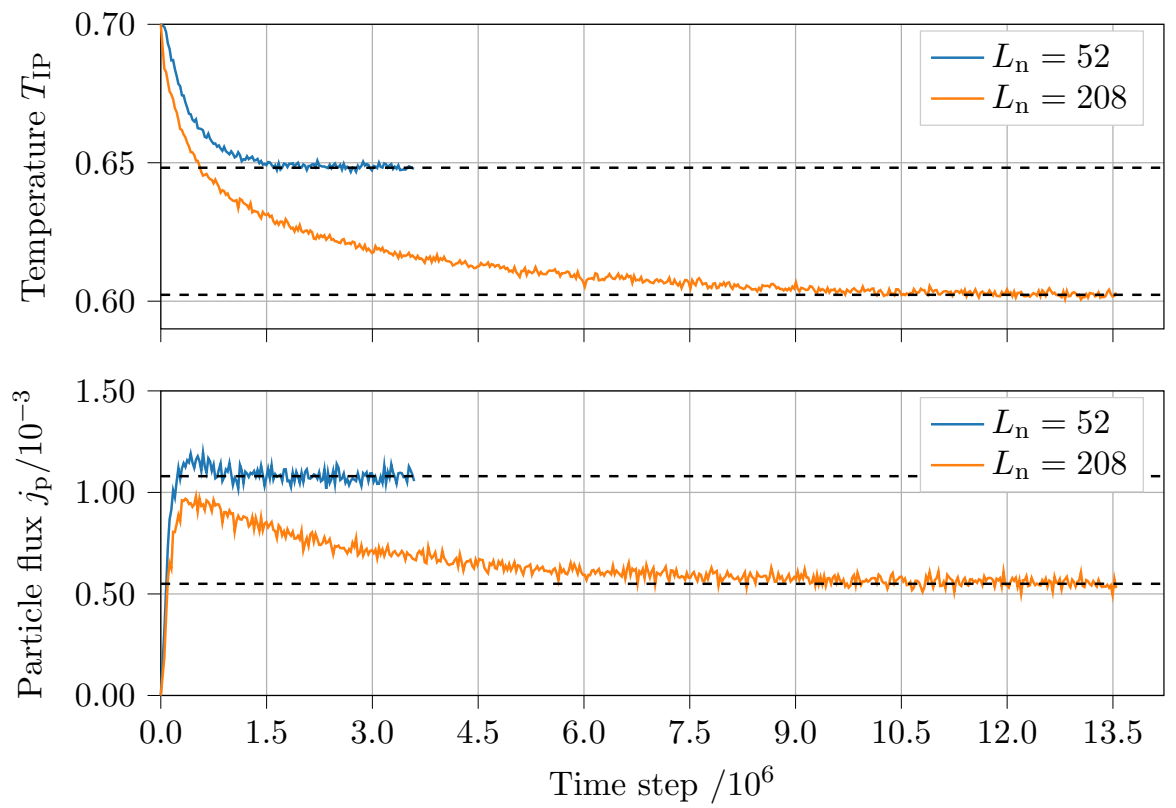

Figure 2. Interface temperature $T_{\mathrm{IP}}$ and particle flux $j_{\mathrm{p}}$ over time steps for $T_{\text {liq }}=0.7$ as well as two different lengths $L_{\mathrm{n}}=52$ and $L_{\mathrm{n}}=208$. Corresponding values under stationary conditions are marked by dashed horizontal lines.

the largest considered length $L_{\mathrm{n}}=208$, this took more than $1.1 \cdot 10^{7}$ time steps. Fig. 2 shows the interface temperature and the particle flux over simulation time steps for two exemplary simulation runs. It can be seen that all values converged, even though it took about four to five times more time for the quantities in the large simulation with $L_{\mathrm{n}}=208$ to reach their respective stationary values. Data given hereafter are averaged simulation results after stationarity was attained.

The time dependence of $T_{\mathrm{IP}}$ and $j_{\mathrm{p}}$ depicted in Fig. 2 shows an exponential asymptotic behavior. Hence, if needed, an according fit function could be used to estimate those values under stationary conditions by extrapolation to $t \rightarrow \infty$.

In order to compare the results of this work with Ref. [41], which relied on another simulation method, we checked whether the outcomes of both techniques are consistent. For this purpose, simulations with the present insertion method were conducted for $T_{\text {liq }}=0.8$ and varying length $L_{\mathrm{n}}$. In one simulation with $L_{\mathrm{n}}=26$, the same vapor length $L_{\mathrm{vap}}=52$ as in Ref. [41] was used, while in the other ones, the vapor length was extended to $L_{\mathrm{vap}}=400$, which was specified also for the remaining simulations of this work. Comparing the results of the simulations with $L_{\mathrm{n}}=26$, the respective temperature profiles of the three simulations are depicted in Fig. 3. Mainly the liquid region is shown as it is in the focus of the present work. The temperature profiles of both simulations with $L_{\mathrm{vap}}=52$ coincide very well and are thus independent of the employed method. This also holds for the simulation with $L_{\mathrm{vap}}=400$, except for the temperature $T_{z}$ in the region of $z>4$. This deviation can be explained by backscattering in the vapor, which practically has the same effect as evaporation against counter-pressure. In Ref. [43], it was shown that a higher counter-pressure leads to a lower hydrodynamic velocity of the vapor flow and vice versa. Accordingly, for the smaller vapor phase length $L_{\text {vap }}=52$ a higher hydrodynamic velocity was found because of less backscattering compared to the case $L_{\text {vap }}=400$. Consequently, a larger fraction of the particles' kinetic energy that constitute the vapor flow contributed to 

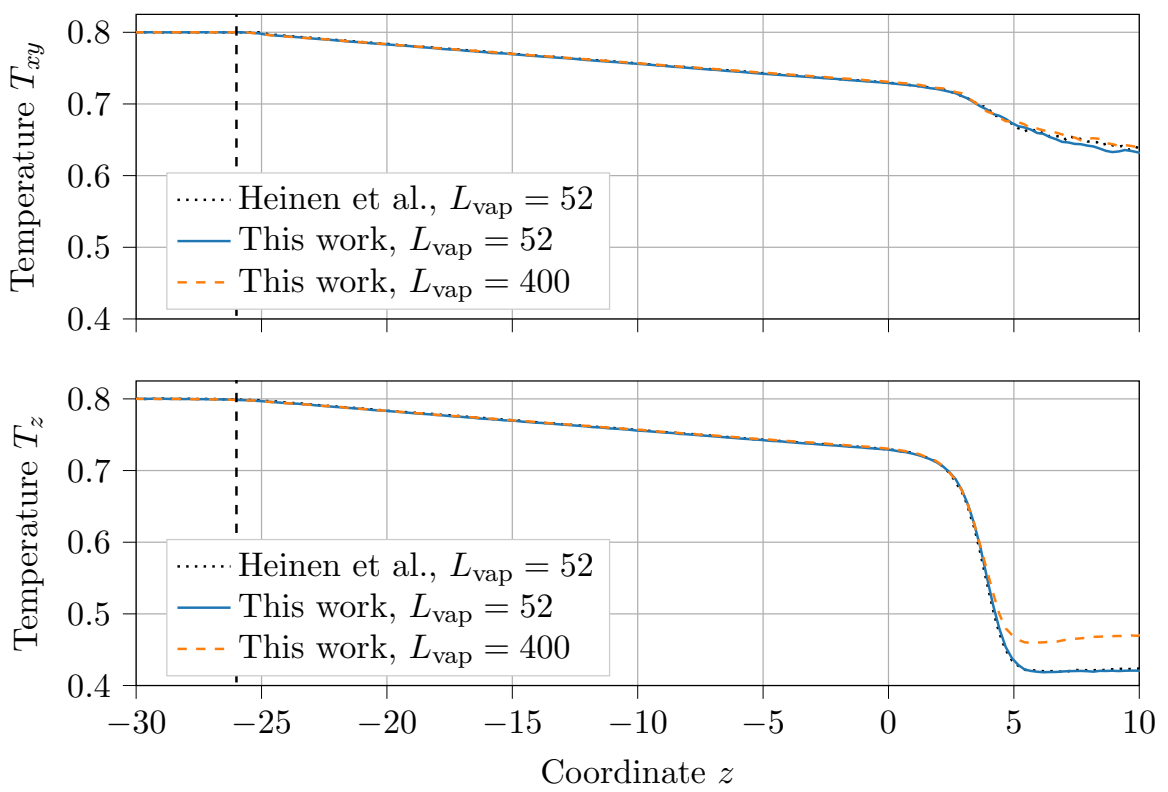

Figure 3. Comparison of temperature profiles $T_{x y}$ and $T_{z}$ from Heinen et al. [41] with those obtained by the present simulation method. The dashed vertical line at $z=-26$ marks the boundary between the thermostated and non-thermostated liquid region.

Table 1. Interface temperature $T_{\mathrm{IP}}$ and particle flux $j_{\mathrm{p}}$ of simulations with varying vapor length $L_{\mathrm{vap}}$ for $T_{\text {liq }}=0.8$ and varying length of the non-thermostated liquid region $L_{\mathrm{n}}$ utilizing the present simulation method in comparison with results of Heinen et al. [41].

\begin{tabular}{lrrrr}
\hline Source & $L_{\mathrm{n}}$ & $L_{\mathrm{vap}}$ & $T_{\mathrm{IP}}$ & $j_{\mathrm{p}} \cdot 10^{3}$ \\
\hline Heinen et al. [41] & 26 & 52 & 0.7251 & 2.856 \\
This work & 26 & 52 & 0.7249 & 2.852 \\
This work & 26 & 400 & 0.7276 & 2.770 \\
\hline
\end{tabular}

the hydrodynamic velocity and not to $T_{z}$. This effect, however, hardly influences the temperature at the interface $T_{\mathrm{IP}}$ and the particle flux $j_{\mathrm{p}}$, cf. Table 1 . Incidentally, in a recent work [74] a temperature anisotropy was also found under equilibrium.

\section{Particle flux}

In Ref. [41], simulations were performed for $T_{\text {liq }}=0.80$, corresponding to $T_{\text {liq }} / T_{\mathrm{c}}=$ 0.74. Data were given for the temperature profiles $T, T_{x y}$ and $T_{z}$, density profile $\rho$, interface temperature $T_{\mathrm{IP}}$ and particle flux $j_{\mathrm{p}}$ for varying length of the non-thermostated liquid region $L_{\mathrm{n}}$. Moreover, extrapolations were made to estimate the interface temperature $T_{\mathrm{IP}}^{\infty}$ and the particle flux $j_{\mathrm{p}}^{\infty}$ for $L_{\mathrm{n}} \rightarrow \infty$.

In the present work, we extend these investigations to the temperatures $T_{\text {liq }}=$ 0.625 (0.579), 0.65 (0.602), $0.70(0.648), 0.75$ (0.694) and $0.80(0.741)$, where the numbers in parentheses denote $T_{\text {liq }} / T_{\mathrm{c}}$. Throughout, the same variation of the length of the non-thermostated liquid region $L_{\mathrm{n}}$ was used as in Ref. [41], i.e. $L_{\mathrm{n}}=5.2$, 15.6, 26, 52, 104 and 208. For the two lowest temperatures, simulations were also conducted with $L_{\mathrm{n}}=10.4$ and 20.8. The quantities sampled with these simulations are the kinetic temperature components parallel to the interface $T_{x y}=T_{x}=T_{y}$, the component perpendicular to the interface $T_{z}$ as well as the average kinetic temperature 

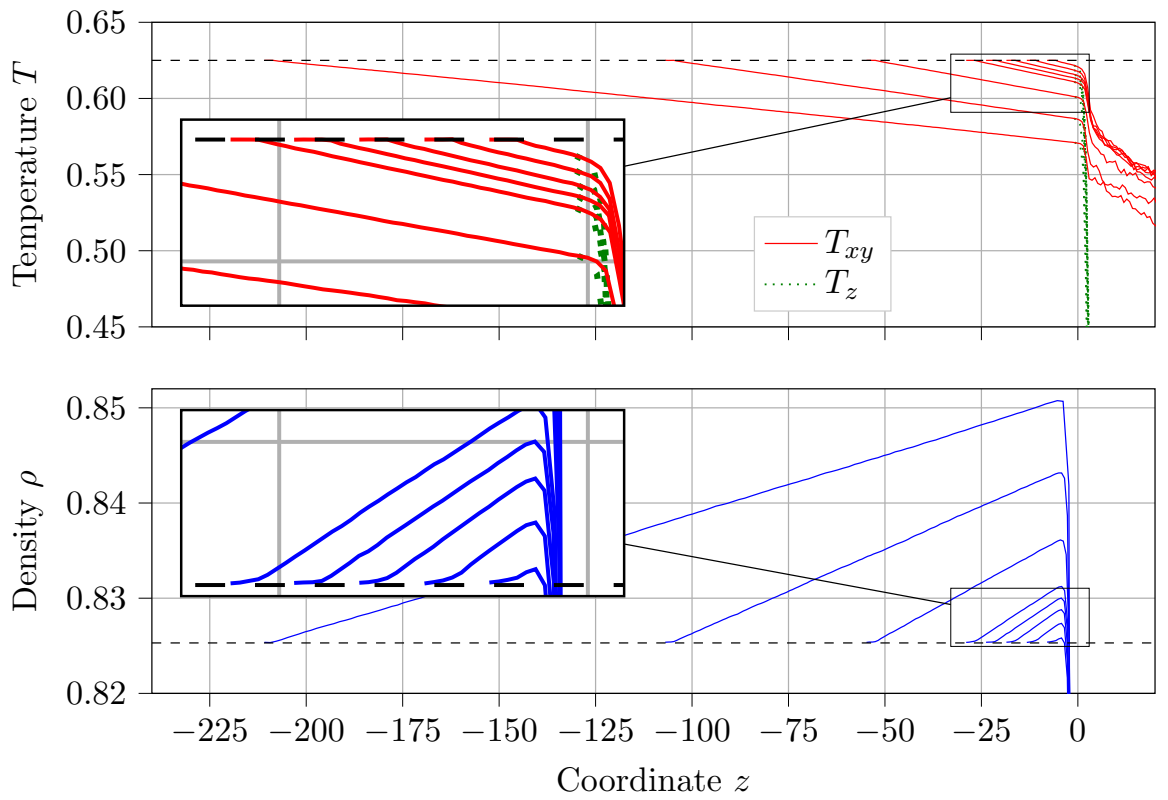

Figure 4. Temperature and density profiles for $T_{\text {liq }}=0.625$ and varying length of the non-thermostated liquid region $L_{\mathrm{n}}$. The horizontal dashed line indicates the value in the thermostated region.

$T=\left(2 T_{x y}+T_{z}\right) / 3$, density $\rho$, hydrodynamic velocity $v_{z}$ and particle flux in positive $z$ direction $j_{\mathrm{p}}^{+}$as well as in negative $z$ direction $j_{\mathrm{p}}^{-}$. The last two quantities yield the net particle flux $j_{\mathrm{p}}=j_{\mathrm{p}}^{+}+j_{\mathrm{p}}^{-}$, which was generally evaluated by the product $j_{\mathrm{p}}=\rho v_{z}$. For convenience, $j_{\mathrm{p}}^{+}$and $j_{\mathrm{p}}^{-}$will be addressed by the terms forward and backward particle flux, in the following.

Once evaporation is stationary, the net particle flux $j_{\mathrm{p}}$ is constant, i.e. it does not depend on the sampling position. Contrary to this, the forward and backward particle fluxes do depend on the sampling position. Since both quantities constitute the backflux ratio $(\mathrm{BFR})-j_{\mathrm{p}}^{-} / j_{\mathrm{p}}^{+}$, it is desired to sample them close to the interface. However, at the IP of the density profile large gradients of density, velocity and, thus particle fluxes occur, cf. Fig. 1, which made it difficult to determine their accurate values at the IP. Hence, the particle fluxes $j_{\mathrm{p}}^{+}$and $j_{\mathrm{p}}^{-}$were sampled at the position of the force extremum plus a distance of 5 into the vapor. This position is sufficiently close to the IP to provide precise values, but distant enough to avoid the aforementioned gradients.

Fig. 4 shows the temperature and density profiles from the bulk liquid over the nonthermostated liquid and the interface to the beginning of the vapor phase for the lowest bulk liquid temperature $T_{\text {liq }}=0.625$ and varying length of the non-thermostated liquid region $L_{\mathrm{n}}$. Within the non-thermostated liquid region, a linear temperature profile was found, connecting the bulk liquid temperature $T_{\text {liq }}$ and interface temperature $T_{\mathrm{IP}}$ by a straight line. According to the temperature decrease towards the interface, a linearly rising density was observed. These findings are conform with those of Heinen et al. [41].

According to our understanding of evaporation, the physical quantities which can be most reliably measured in the lab are the bulk liquid temperature and the particle flux. Hence, our main interest was to obtain results for the particle flux $j_{\mathrm{p}}$ for several bulk liquid temperatures $T_{\text {liq }}$ and varying length of the non-thermostated liquid region $L_{\mathrm{n}}$. A macroscopically long non-thermostated liquid region means $L_{\mathrm{n}} \rightarrow \infty$ on a molecular 
Table 2. Results of the entire simulation series with varying bulk liquid temperature $T_{\text {liq }}$ and length of the non-thermostated liquid region $L_{\mathrm{n}}$ for interface temperature $T_{\mathrm{IP}}$, forward and backward particle fluxes $j_{\mathrm{p}}^{+}$and $j_{\mathrm{p}}^{-}$, BFR, net particle flux $j_{\mathrm{p}}$, Hertz flux $j^{\mathrm{H}}$ and evaporation coefficient $\alpha$.

\begin{tabular}{|c|c|c|c|c|c|c|c|c|}
\hline$T_{\text {liq }}$ & $L_{\mathrm{n}}$ & $T_{\mathrm{IP}}$ & $j_{\mathrm{p}}^{+} \cdot 10^{3}$ & $j_{\mathrm{p}}^{-} \cdot 10^{3}$ & $-j_{\mathrm{p}}^{-} / j_{\mathrm{p}}^{+}$ & $j_{\mathrm{p}} \cdot 10^{3}$ & $j^{\mathrm{H}} \cdot 10^{3}$ & $\alpha$ \\
\hline \multirow[t]{9}{*}{0.625} & 5.2 & 0.6205 & 0.840 & -0.113 & 0.134 & 0.727 & 0.933 & 0.780 \\
\hline & 10.4 & 0.6177 & 0.810 & -0.112 & 0.138 & 0.698 & 0.933 & 0.749 \\
\hline & 15.6 & 0.6149 & 0.768 & -0.103 & 0.134 & 0.665 & 0.933 & 0.713 \\
\hline & 20.8 & 0.6123 & 0.736 & -0.098 & 0.133 & 0.638 & 0.933 & 0.684 \\
\hline & 26 & 0.6105 & 0.720 & -0.095 & 0.132 & 0.625 & 0.933 & 0.670 \\
\hline & 52 & 0.6007 & 0.627 & -0.082 & 0.130 & 0.545 & 0.933 & 0.585 \\
\hline & 104 & 0.5860 & 0.484 & -0.058 & 0.120 & 0.426 & 0.933 & 0.457 \\
\hline & 208 & 0.5702 & 0.368 & -0.044 & 0.119 & 0.325 & 0.933 & 0.348 \\
\hline & $\infty$ & 0.559 & 0.314 & -0.036 & 0.115 & 0.278 & 0.933 & 0.298 \\
\hline \multirow[t]{9}{*}{0.65} & 5.2 & 0.6439 & 1.173 & $\begin{array}{l}-0.168 \\
\end{array}$ & 0.143 & 1.005 & 1.294 & 0.777 \\
\hline & 10.4 & 0.6395 & 1.098 & -0.150 & 0.137 & 0.948 & 1.294 & 0.732 \\
\hline & 15.6 & 0.6359 & 1.050 & -0.146 & 0.139 & 0.904 & 1.294 & 0.699 \\
\hline & 20.8 & 0.6323 & 1.002 & -0.141 & 0.141 & 0.861 & 1.294 & 0.665 \\
\hline & 26 & 0.6298 & 0.963 & -0.133 & 0.138 & 0.830 & 1.294 & 0.641 \\
\hline & 52 & 0.6174 & 0.812 & -0.108 & 0.133 & 0.705 & 1.294 & 0.544 \\
\hline & 104 & 0.6006 & 0.613 & -0.078 & 0.128 & 0.535 & 1.294 & 0.413 \\
\hline & 208 & 0.5815 & 0.453 & -0.055 & 0.121 & 0.398 & 1.294 & 0.307 \\
\hline & $\infty$ & 0.569 & 0.395 & -0.047 & 0.119 & 0.348 & 1.294 & 0.269 \\
\hline \multirow[t]{7}{*}{0.7} & 5.2 & 0.6895 & 2.095 & -0.308 & 0.147 & 1.787 & 2.441 & 0.732 \\
\hline & 15.6 & 0.6762 & 1.797 & -0.258 & 0.143 & 1.539 & 2.441 & 0.630 \\
\hline & 26 & 0.6663 & 1.588 & -0.228 & 0.144 & 1.359 & 2.441 & 0.557 \\
\hline & 52 & 0.6484 & 1.258 & -0.173 & 0.138 & 1.085 & 2.441 & 0.444 \\
\hline & 104 & 0.6261 & 0.921 & -0.129 & 0.140 & 0.793 & 2.441 & 0.325 \\
\hline & 208 & 0.6023 & 0.625 & -0.081 & 0.130 & 0.544 & 2.441 & 0.223 \\
\hline & $\infty$ & 0.589 & 0.563 & -0.069 & 0.126 & 0.494 & 2.441 & 0.202 \\
\hline \multirow[t]{7}{*}{0.75} & 5.2 & 0.7312 & 3.439 & -0.518 & 0.151 & 2.921 & 4.290 & 0.681 \\
\hline & 15.6 & 0.7125 & 2.774 & -0.417 & 0.150 & 2.358 & 4.290 & 0.550 \\
\hline & 26 & 0.6989 & 2.363 & -0.354 & 0.150 & 2.009 & 4.290 & 0.468 \\
\hline & 52 & 0.6755 & 1.789 & -0.264 & 0.147 & 1.526 & 4.290 & 0.356 \\
\hline & 104 & 0.6480 & 1.245 & -0.177 & 0.142 & 1.068 & 4.290 & 0.249 \\
\hline & 208 & 0.6192 & 0.827 & -0.110 & 0.134 & 0.716 & 4.290 & 0.167 \\
\hline & $\infty$ & 0.608 & 0.787 & -0.096 & 0.122 & 0.691 & 4.290 & 0.161 \\
\hline \multirow[t]{8}{*}{0.8} & 5.2 & 0.7717 & 5.153 & -0.783 & 0.152 & 4.370 & 7.059 & 0.619 \\
\hline & 10.4 & 0.7572 & 4.434 & -0.676 & 0.152 & 3.758 & 7.059 & 0.532 \\
\hline & 15.6 & 0.7448 & 3.914 & -0.591 & 0.151 & 3.324 & 7.059 & 0.471 \\
\hline & 26 & 0.7276 & 3.265 & -0.494 & 0.151 & 2.770 & 7.059 & 0.392 \\
\hline & 52 & 0.6983 & 2.333 & -0.344 & 0.148 & 1.989 & 7.059 & 0.282 \\
\hline & 104 & 0.6649 & 1.556 & -0.226 & 0.145 & 1.330 & 7.059 & 0.188 \\
\hline & 208 & 0.6218 & 1.017 & -0.142 & 0.139 & 0.875 & 7.059 & 0.124 \\
\hline & $\infty$ & 0.628 & 0.982 & -0.138 & 0.141 & 0.844 & 7.059 & 0.120 \\
\hline
\end{tabular}




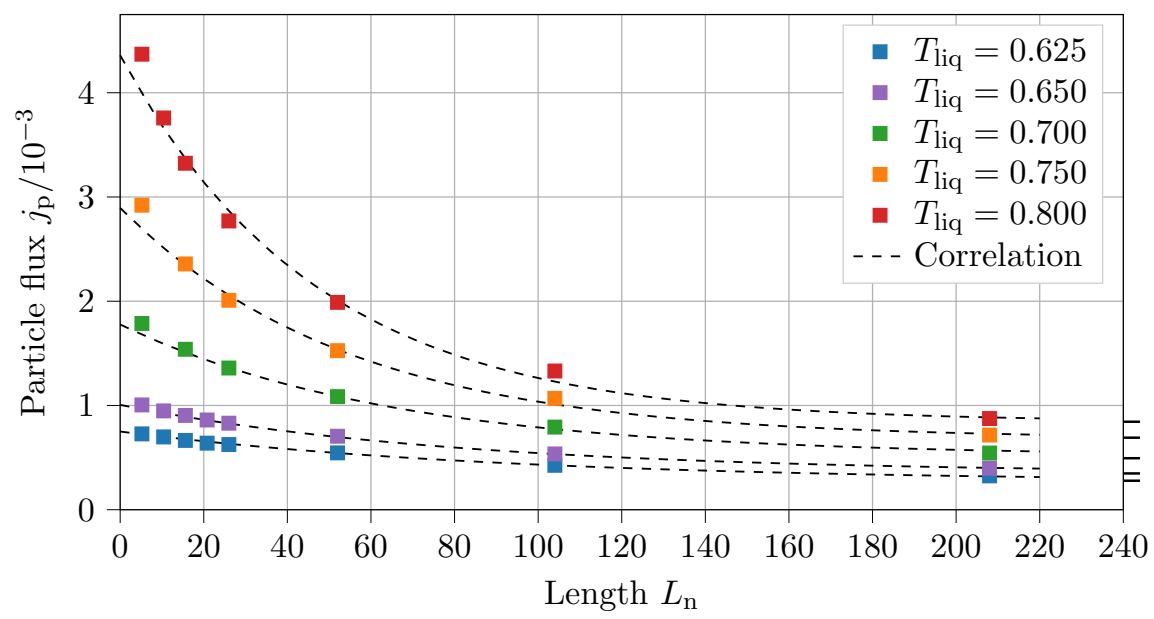

Figure 5. Particle flux $j_{\mathrm{p}}$ over length of the non-thermostated liquid region $L_{\mathrm{n}}$ for varying bulk liquid temperature $T_{\text {liq. }}$. The dashed lines show correlation (3), while the dashes on the right vertical axis indicate the particle flux $j_{\mathrm{p}}^{\infty}$ for $L_{\mathrm{n}} \rightarrow \infty$.

scale, which leads to a limiting value $j_{\mathrm{p}}^{\infty}$. As the fluxes in Table 2 show an exponential asymptotic behavior, a correlation of the type

$$
j_{\mathrm{p}}=j_{\mathrm{p}}^{\infty}+f \exp \left(-g L_{\mathrm{n}}\right),
$$

with temperature dependent coefficients $j_{\mathrm{p}}^{\infty}, f$ and $g$ was fitted to the data yielding

$$
\begin{aligned}
j_{\mathrm{p}}^{\infty} \cdot 10^{3} & =-1.79+3.29 T_{\text {liq }}, \\
f \cdot 10^{3} & =+24.3-81.5 T_{\text {liq }}+69.4 T_{\text {liq }}^{2}, \\
g \cdot 10^{3} & =-26.9+60.1 T_{\text {liq }} .
\end{aligned}
$$

Particle flux data from MD together with correlation (3) are shown in Fig. 5, where the dashes on the right vertical axis indicate the values for $j_{\mathrm{p}}^{\infty}$.

Of particular interest is the evaporation coefficient $\alpha$ that is the ratio of the particle flux $j_{\mathrm{p}}$ and the Hertz flux $j^{\mathrm{H}}$ corresponding to a reference temperature $T$

$$
j^{\mathrm{H}}(T)=\rho^{\prime \prime}(T) \sqrt{\frac{k_{\mathrm{B}} T}{2 \pi m}},
$$

with $\rho^{\prime \prime}$ being the saturated vapor density and $k_{\mathrm{B}}$ the Boltzmann constant. Care should be taken in distinguishing different particle fluxes and different reference temperatures. According to our understanding, Hertz [1] initially meant the flux of outgoing particles $j_{\mathrm{p}}^{+}$and the bulk liquid temperature, corresponding to

$$
\alpha^{+}=j^{+} / j^{\mathrm{H}}\left(T_{\text {liq }}\right)
$$

As the flux of outgoing particles $j_{\mathrm{p}}^{+}$is difficult to determine experimentally, the net 


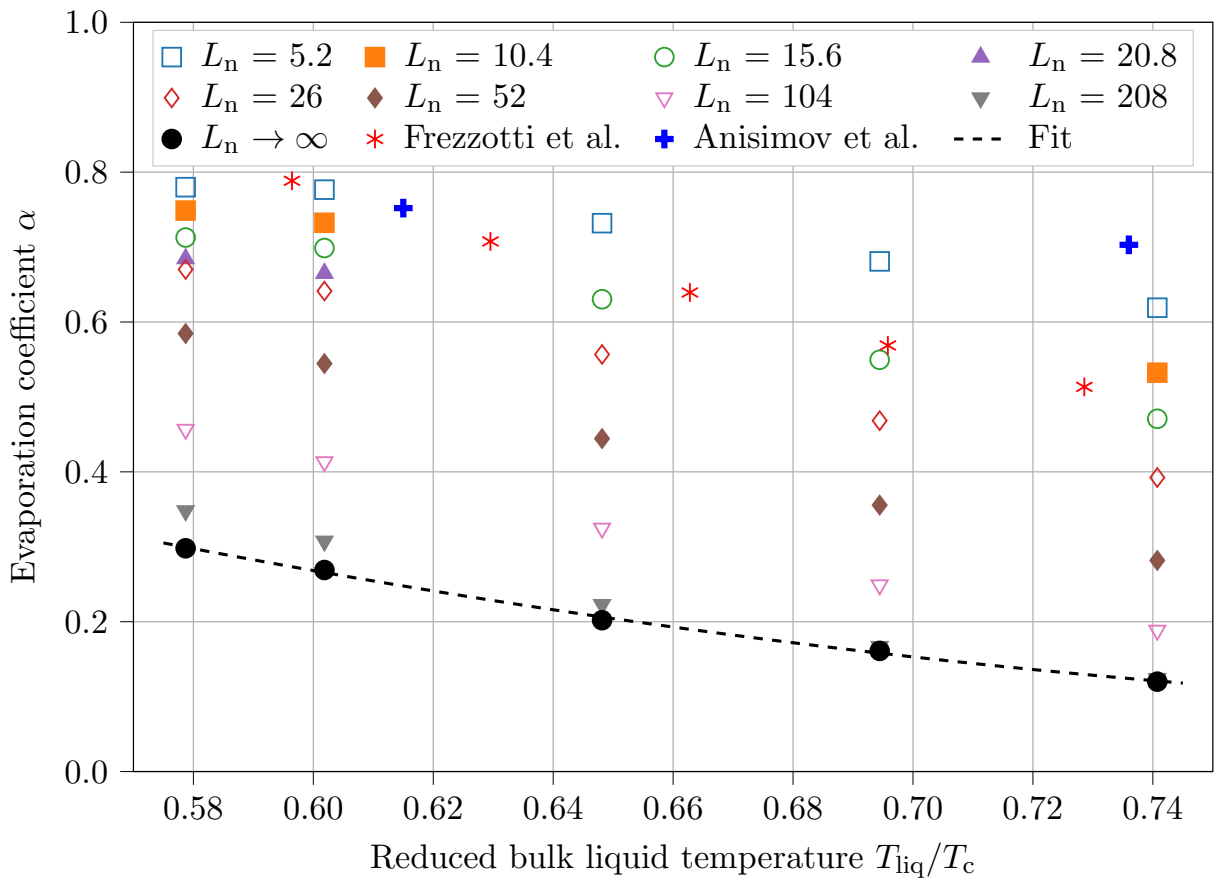

Figure 6. Evaporation coefficient $\alpha$ over reduced bulk liquid temperature $T_{\text {liq }} / T_{\mathrm{c}}$ for varying length of the non-thermostated liquid region $L_{\mathrm{n}}$ in comparison with results of Frezzotti et al. [20] $\left(L_{\mathrm{n}} \approx 16\right)$ and Anisimov et al. [28] $\left(L_{\mathrm{n}} \approx 10\right)$. The dashed line through the data points for $L_{\mathrm{n}} \rightarrow \infty$ serves as a guide to the eye.

particle flux $j_{\mathrm{p}}$ is frequently used to define the evaporation coefficient

$$
\alpha=j_{\mathrm{p}} / j^{\mathrm{H}}\left(T_{\text {liq }}\right) .
$$

Results for $\alpha$ obtained from the sampled net particle flux as well as from the extrapolated flux $j_{\mathrm{p}}^{\infty}$ are given in the last column of Table 2. A graphical representation of the evaporation coefficient for varying length of the non-thermostated liquid region $L_{\mathrm{n}}$ and reduced liquid bulk temperature $T_{\text {liq }} / T_{\mathrm{c}}$ is given in Fig. 6 .

Fig. 6 illustrates the strong dependence of the evaporation coefficient $\alpha$ on the length of the non-thermostated liquid region $L_{\mathrm{n}}$, which is an essential message of the previous communication [41] and the present article. The figure also shows the temperature dependence of $\alpha\left(L_{\mathrm{n}} \rightarrow \infty\right)$. For comparison, Fig. 6 contains MD results by Anisimov et al. [28] using $L_{\mathrm{n}} \approx 10$ and KEF data of Frezzotti et al. [20] with $L_{\mathrm{n}} \approx 16$. It can be seen that the good agreement between the results of Ref. [20] and the present MD results holds for the entire temperature range.

In Ref. [41], we also addressed the dependence of the interface temperature on the length of the non-thermostated liquid region $L_{\mathrm{n}}$ and the dependence of the particle flux on the interface temperature $T_{\mathrm{IP}}$. Simulation results for $T_{\mathrm{IP}}$ as a function of $T_{\mathrm{liq}}$ and $L_{\mathrm{n}}$ are listed in Table 2 and shown in Fig. 7.

In Ref. [41], we suggested a correlation for the interface temperature which allows for the extrapolation to $L_{\mathrm{n}} \rightarrow \infty$

$$
T_{\mathrm{IP}}=T_{\mathrm{IP}}^{\infty}+b \exp \left(-c L_{\mathrm{n}}\right)
$$




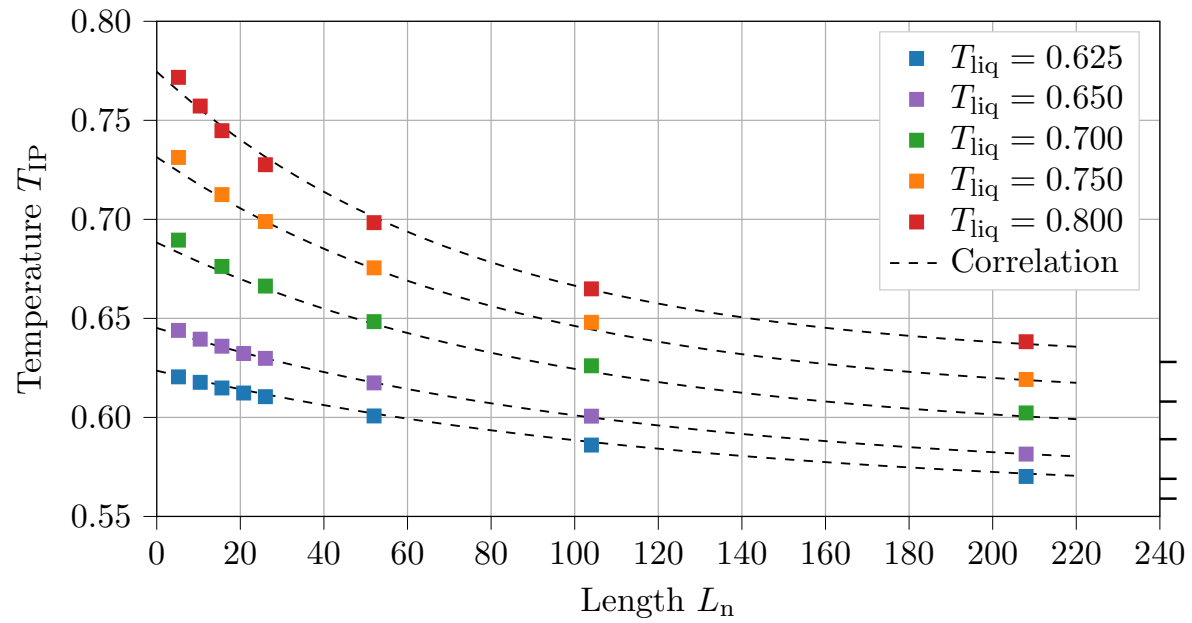

Figure 7. Interface temperature $T_{\mathrm{IP}}$ over length of the non-thermostated liquid region $L_{\mathrm{n}}$ for varying bulk liquid temperature $T_{\text {liq. }}$. The dashed lines show correlation (8) with temperature dependent coefficients given in Eqs. (9). The dashes on the right vertical axis indicate the interface temperature for $L_{\mathrm{n}} \rightarrow \infty$.

This correlation can be generalized to the present range of the bulk liquid temperature $T_{\text {liq }}$ with linear functions for its coefficients

$$
\begin{aligned}
T_{\mathrm{IP}}^{\infty}=+0.313 & +0.394 T_{\text {liq }}, \\
b & =-0.228+0.469 T_{\text {liq }}, \\
c & =-0.0118+0.0315 T_{\text {liq }} .
\end{aligned}
$$

The dashes on the right vertical axis in Fig. 7 indicate the values for $T_{\mathrm{IP}}^{\infty}$. The dependence of the particle flux $j_{\mathrm{p}}$ on the interface temperature $T_{\mathrm{IP}}$ is shown in Fig. 8. All simulation results of this work are contained and it can be seen that they approximately lie on a single curve which can be represented by

$$
j_{\mathrm{p}}=A_{\mathrm{p}}+B_{\mathrm{p}} \exp \left(C_{\mathrm{p}} T_{\mathrm{IP}}\right)
$$

with $A_{\mathrm{p}}=-4.394 \cdot 10^{-4}, B_{\mathrm{p}}=3.524 \cdot 10^{-6}$ and $C_{\mathrm{p}}=9.360$. Fig. 8 confirms the statement given in Ref. [41] that the evaporation flux $j_{\mathrm{p}}$ in essence depends only on the interface temperature $T_{\mathrm{IP}}$. While this statement in Ref. [41] was based only on three data sets at $T_{\mathrm{IP}} / T_{\mathrm{c}} \approx 0.74$, now 35 data sets ranging from $T_{\mathrm{IP}} / T_{\mathrm{c}} \approx 0.54$ to 0.74 were considered.

A classical question concerns the aforementioned BFR, which is also given in Table 2 . In order to gather additional information about the BFR at $T_{\text {liq }}=0.8$, those simulations had to be carried out again, since relevant data were not given in Ref. [41]. Results obtained from kinetic theory of gases are $18 \%$ [75], $15 \%$ [76] or $16.2 \%$ [77] and independent on temperature. Based on MD simulations, Lotfi [26] reported for a length of the vapor phase $L_{\mathrm{vap}} \approx 80$ temperature dependent BFR of $5 \%$ for $T_{\text {liq }} / T_{\mathrm{c}}=0.53,7 \%$ for $T_{\text {liq }} / T_{\mathrm{c}}=0.64$ and $9 \%$ for $T_{\text {liq }} / T_{\mathrm{c}}=0.83$. Since $j_{\mathrm{p}}$ was found to be a function of the interface temperature $T_{\mathrm{IP}}$, cf. Fig. 8 and Eq. (10), also the BFR only depends on $T_{\mathrm{IP}}$, as can be seen in Fig. 9. 


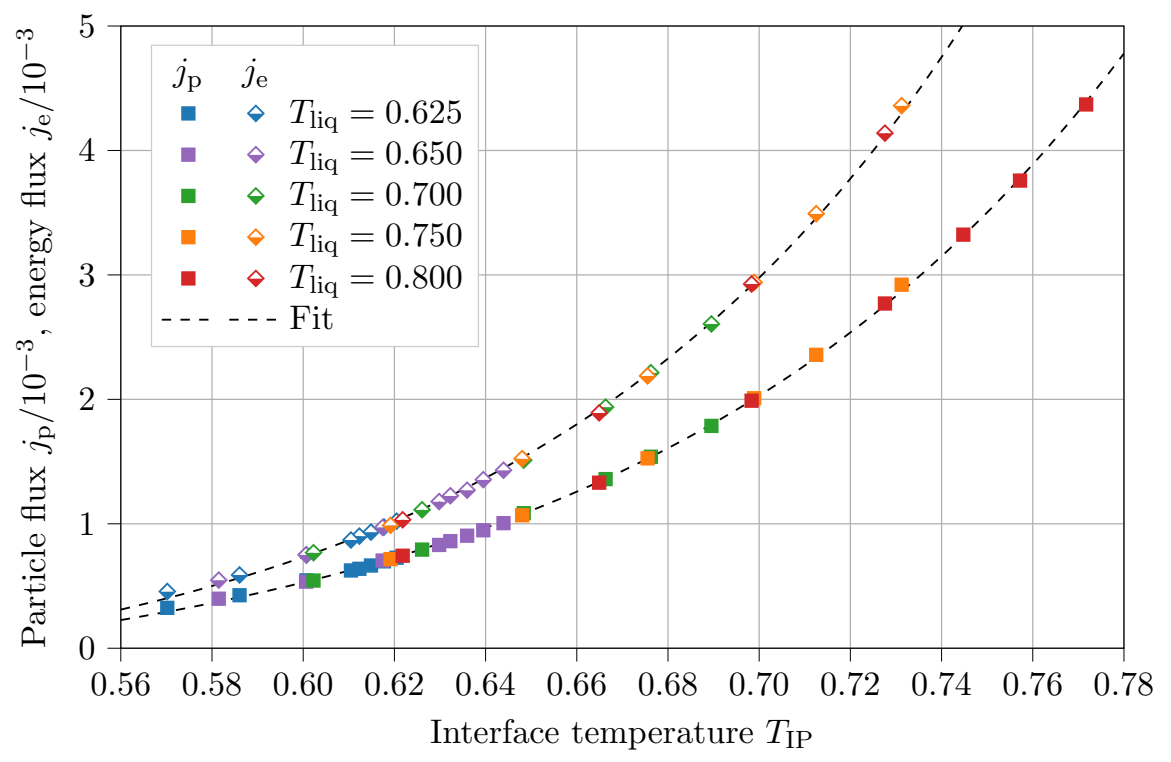

Figure 8. Particle flux $j_{\mathrm{p}}$ and energy flux $j_{\mathrm{e}}$ over interface temperature $T_{\mathrm{IP}}$ for varying bulk liquid temperature $T_{\text {liq }}$ and length of the non-thermostated liquid region $L_{\mathrm{n}}$. For a given value of $T_{\text {liq }}, L_{\mathrm{n}}$ increases from right to left. The dashed lines indicate the fits (10) and (13).

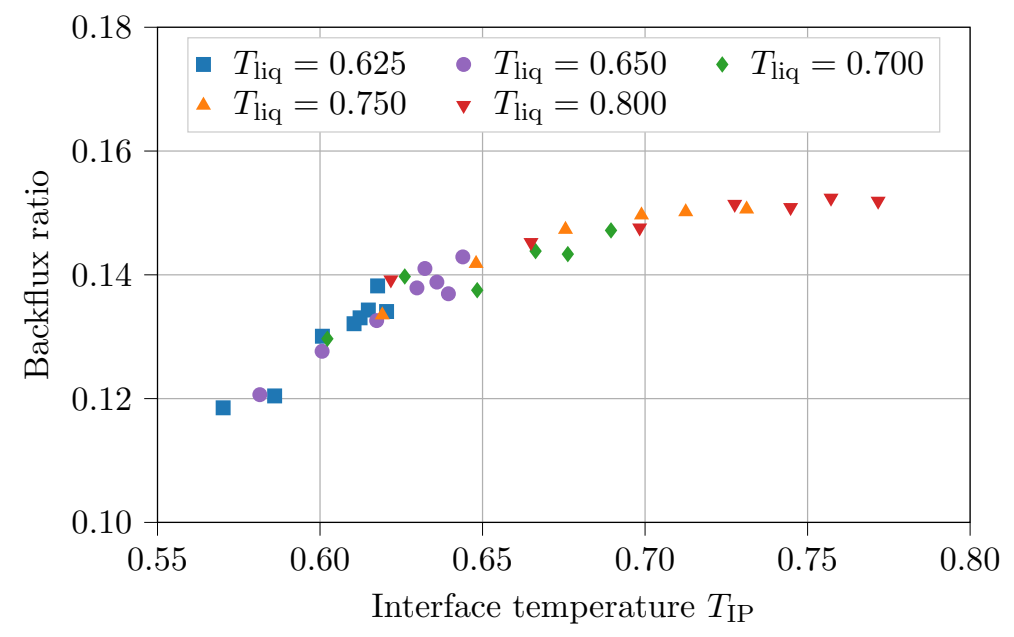

Figure 9. Backflux ratio over interface temperature $T_{\mathrm{IP}}$ for varying bulk liquid temperature $T_{\text {liq }}$ and length of the non-thermostated liquid region $L_{\mathrm{n}}$. For a given value of $T_{\text {liq }}, L_{\mathrm{n}}$ increases from right to left. 
Table 3. Evaporation coefficient $\alpha$ from experiment [82] compared with MD results obtained in this work.

\begin{tabular}{lrrrr}
\hline Fluid & $T_{\mathrm{c}}(\mathrm{K})$ & $T_{\mathrm{IP}}(\mathrm{K})$ & $T_{\mathrm{IP}} / T_{\mathrm{c}}$ & $\alpha$ \\
\hline Toluene & 593 & 283 & 0.477 & $0.45-0.83$ \\
Benzene & 562 & 280 & 0.498 & $0.86-0.93$ \\
LJTS2.5 & & & 0.54 & 0.30 \\
LJTS2.5 & & & 0.59 & 0.12 \\
\hline
\end{tabular}

The essential message from the present MD results and those from Ref. [41] is the large temperature drop from the bulk liquid to the interface that is necessary for heat transport. This temperature drop must be taken into account when formulating kinetic boundary conditions.

The real challenge for evaporation studies is the comparison of the evaporation coefficient $\alpha$ from theories or simulations with experimental data. As mentioned in the introduction, several compilations of experimental $\alpha$ values are available for different fluids [60, 61], including water [62, 63]. The first impression from these compilations is that $\alpha$ values for water with stagnant interfaces are below 0.1 [63] and similarly small $\alpha$ values were obtained for other fluids with hydrogen bonds, like methanol or ethanol [60]. Concerning this matter, we compared the enthalpy of evaporation of three fluids with hydrogen bonds, i.e. water, methanol and ethanol, to three fluids without hydrogen bonds, i.e. hexane, benzene and carbon tetrachloride. The enthalpy of evaporation of fluids with hydrogen bonds is substantially larger than that of fluids without hydrogen bonds. Hence, it may be concluded that strong hydrogen bonds in the liquid prevent higher evaporation rates. As model potentials like LJ or LJTS2.5 do not create hydrogen bonds, a comparison of the present results with water does not make much sense. One should rather look at fluids that can be described by LJ, n-center LJ or similar interaction potentials, like carbon tetrachloride [78], benzene [79] or toluene [80].

First, carbon tetrachloride with $T_{\mathrm{c}}=556.4 \mathrm{~K}$ for which $\alpha$ was experimentally studied by Prüger [4] and Bogdandy et al. [81] is considered. The measurements in Ref. [4] were made under atmospheric pressure at the temperature $T=350 \mathrm{~K}$, hence $T / T_{\mathrm{c}}=0.63$, and gave $\alpha=1$. The measurements in Ref. [81] were made at $273.15 \mathrm{~K}$, i.e. $T / T_{\mathrm{c}}=0.49$, and gave $\alpha=0.99$. It is somewhat surprising that the experimental $\alpha$ values do not show a significant temperature dependence and in any case, there is a remarkable difference between the experimental results and the values from KEF and MD.

Next, $\alpha$ values of benzene and toluene, which were measured by Baranaev [82] and are reported in Refs. [60, 83], were taken into account. The temperature given there is an estimated interface temperature, which we assume to be $T_{\mathrm{IP}}$. The interface temperature $T_{\mathrm{IP}}$, its reduced form $T_{\mathrm{IP}} / T_{\mathrm{c}}$ and experimental $\alpha$ values are listed in Table 3 together with results of the present MD simulations. This comparison indicates only some similarity between experimental and MD results.

\section{Heat flux}

The particle flux's strong dependence on the interface temperature $T_{\mathrm{IP}}$ was thoroughly discussed in the previous section. Moreover, it was shown that $T_{\mathrm{IP}}$ can be correlated on the basis of the parameters $T_{\text {liq }}$ and $L_{\mathrm{n}}$, as they govern the heat transport through the liquid. This section contains a detailed analysis of the energy flux $j_{\mathrm{e}}$ through the sim- 
ulation volume, including the heat flux $\dot{q}$, to study the contributions of convective and diffusive energy transport. Buoyancy driven convection and radiation can be excluded per definition, since they were not incorporated in the simulation model. Hence, the transport mechanisms were limited to advection, given by the bulk motion of the replenished liquid, and heat conduction that established the heat flux $\dot{q}$, induced by the temperature gradient between the thermostated liquid and the interface. Nevertheless, results of the present investigations should also be comparable to experimental data of macroscopic systems exposed to e.g. earth's gravitational field, i.e. not limited to microgravity environments, since Ref. [10] gives an indication that natural convection is of little importance in microscopically thin liquid layers. Schreiber and Cammenga [10] elucidated that there is a subsurface layer, wherein convective motion is progressively damped. In water, they measured subsurface layer widths of up to two millimeters. Compared to that, subsurface layers less than a hundred nanometers wide that were investigated in the present NEMD simulations are several orders of magnitude smaller.

The energy flux was calculated with a conservative form of the energy equation [84], neglecting the contribution of the viscous stress tensor

$$
j_{\mathrm{e}}=\left(h+e_{\mathrm{kin}}\right) j_{\mathrm{p}}+\dot{q},
$$

where $h$ is the enthalpy and $e_{\text {kin }}$ the kinetic energy of the volume element within a bin, i.e. the term $\left(h+e_{\text {kin }}\right) j_{\mathrm{p}}$ describes the energy transport by advection and the heat flux $\dot{q}$ is the diffusive part that was described by Fourier's law

$$
\dot{q}=-\lambda \mathrm{d} T / \mathrm{d} z,
$$

with the thermal conductivity $\lambda$. The present evaluation is exemplarily shown in Fig. 10 for the case $T_{\text {liq }}=0.75$ and $L_{\mathrm{n}}=104$. All 35 studied conditions are compiled in the supplementary material.

Panel a) depicts profiles that were sampled on the fly, while the quantities in panels b) to f) were obtained by post-processing. From the first derivative of the temperature profile in panel b), together with the thermal conductivity in panel c), the heat flux $\dot{q}$ was calculated with Eq. (12). The thermal conductivity $\lambda$ was determined by using the temperature and density profiles as input values for the correlations by Lautenschläger et al. [85] and Lemmon et al. [86]. The first correlation was used for liquid states and the second one for vapor states. As expected, it can be seen that $\lambda$ increases in the liquid with rising density, while on the vapor side, $\lambda$ is one order of magnitude smaller. Together with a weak temperature gradient, the heat flux $\dot{q}$ in the vapor phase is thus negligible, cf. panel f). Panel d) shows the particle flux $j_{\mathrm{p}}$ that is needed to calculate the advective part of Eq. (11) together with the enthalpy $h$ and the kinetic energy $e_{\text {kin }}$ depicted in panel e). While $e_{\text {kin }}$ could be obtained straightforwardly from the hydrodynamic velocity $v_{z}$, the enthalpy $h(T, \rho)$ was calculated with the LJTS2.5 equation of state proposed by Heier et al. [87], again using the sampled temperature and density profiles as input values. The reference point of the enthalpy was deliberately specified such that at temperature $T_{0}=0.8$ and a vanishing density $\rho_{0}=10^{-6}$ the ideal gas contribution is yielded $h\left(T_{0}, \rho_{0}\right)=h_{0}^{\circ}=c_{p}^{\circ} T_{0}=5 / 2 \cdot 0.8=2$. Note that this reference point differs from that used in Ref. [88], although we used the implementation of the LJTS2.5 equation of state published by Hitz et al. [88]. As expected, the enthalpy profile shows a jump at the interface, reflecting the enthalpy 
of evaporation. On the vapor side, $h$ decreases according to falling temperature and density. But since $e_{\text {kin }}$ increases simultaneously by approximately the same amount, the sum $h+e_{\text {kin }}$ attains an almost constant value throughout the vapor phase. On the liquid side, $e_{\text {kin }}$ is negligible because of the slow hydrodynamic velocity of the liquid.

Finally, panel f) depicts the evaluation of Eq. (11). Looking at the vapor side, the constant value of $h+e_{\text {kin }}$, cf. panel e), multiplied by a constant particle flux $j_{\mathrm{p}}$, cf. panel d), together with a negligible heat flux $\dot{q}$, yields a constant energy flux $j_{\mathrm{e}}$. On the liquid side, the flat profiles to the very left indicate the thermostated liquid with $\dot{q}=0$ because of the constant temperature $T_{\text {liq }}$. Therein, the kinetic energy continuously added by the thermostat balances the heat flux $\dot{q}$ at the boundary of the thermostated region at $z=-104$. Following those profiles to the right, the enthalpy $h$ decreases with approximately the same slope with that the heat flux $\dot{q}$ increases within the non-thermostated liquid region so that the sum of the advective term $\left(h+e_{\mathrm{kin}}\right) j_{\mathrm{p}}$ and the diffusive term $\dot{q}$ of Eq. (12) also yields an almost constant value. Accordingly, from liquid to vapor, an overall flat energy flux profile was obtained, indicating that stationary energy balance was fulfilled. Hence, a global value for $j_{\mathrm{e}}$ was determined for each of the 35 simulation runs. Since $j_{\mathrm{p}}$ and $j_{\mathrm{e}}$ are more difficult to sample within the liquid phase, i.e. they are associated with larger uncertainties because of the very low hydrodynamic velocity, the global value of $j_{\mathrm{e}}$ was determined in the vapor phase. More precisely, it was evaluated at the position where the minimum difference between the transversal and longitudinal temperature $\min \left(T_{x y}-T_{z}\right)$ was found.

Values for $j_{\mathrm{e}}$ acquired along this route from all simulations were plotted over $T_{\mathrm{IP}}$ in Fig. 8. As already seen for $j_{\mathrm{p}}$, also all data points for $j_{\mathrm{e}}$ fall on a single curve that can be described by an exponential fit

$$
j_{\mathrm{e}}=A_{\mathrm{e}}+B_{\mathrm{e}} \exp \left(C_{\mathrm{e}} T_{\mathrm{IP}}\right),
$$

with $A_{\mathrm{e}}=-5.236 \cdot 10^{-4}, B_{\mathrm{e}}=2.688 \cdot 10^{-6}$ and $C_{\mathrm{e}}=10.25$.

This observation reveals a crucial finding: The interface temperature $T_{\mathrm{IP}}$ can be identified as a key quantity in the energy balance, connecting the particle flux $j_{\mathrm{p}}$ and the energy flux $j_{\mathrm{e}}$. It determines not only the heat flux $\dot{q}$ as a result of the established temperature gradient, supplying the interface with the enthalpy of evaporation, but also the particle flux $j_{\mathrm{p}}$ that influences the energy flux $j_{\mathrm{e}}$ according to the advective term of Eq. (11). Hence, as represented by Eqs. (8) and (9), for given boundary conditions, a certain interface temperature $T_{\mathrm{IP}}\left(T_{\text {liq }}, L_{\mathrm{n}}\right)$ exists that yields $j_{\mathrm{e}}=$ const. under stationary conditions. Note that different combinations of $T_{\text {liq }}$ and $L_{\mathrm{n}}$ can yield the same value of $T_{\mathrm{IP}}$, cf. Fig. 7 . For higher interface temperatures $T_{\mathrm{IP}}>T_{\mathrm{IP}}\left(T_{\text {liq }}, L_{\mathrm{n}}\right)$, accompanied by a larger particle flux $j_{\mathrm{p}}$ and a smaller heat flux $\dot{q}$ on the liquid side due to a weaker temperature gradient, the amount of energy carried away from the interface by evaporation is larger than that supplied by the liquid, such that $T_{\mathrm{IP}}$ will decrease over time. For lower interface temperatures $T_{\mathrm{IP}}<T_{\mathrm{IP}}\left(T_{\text {liq }}, L_{\mathrm{n}}\right)$, accompanied by a smaller particle flux $j_{\mathrm{p}}$ and a larger heat flux $\dot{q}$, the situation is vice versa: The amount of energy supplied by the liquid exceeds the one carried away by the vapor, leading to an increase of $T_{\mathrm{IP}}$ until stationary conditions are attained. 


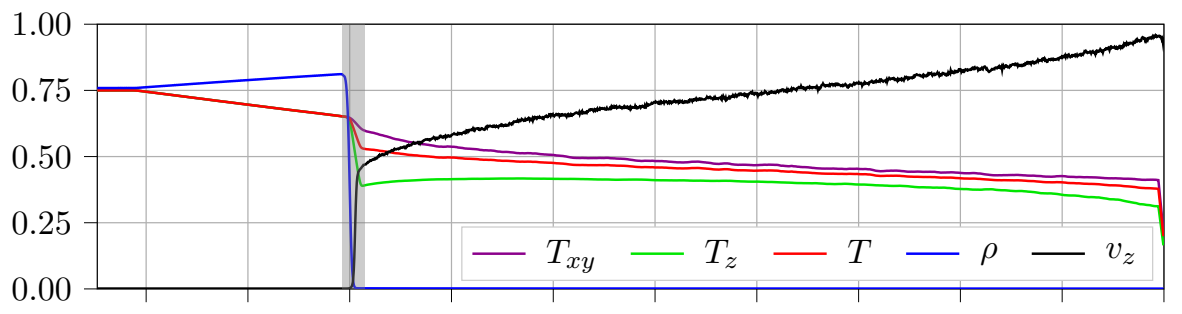

a)
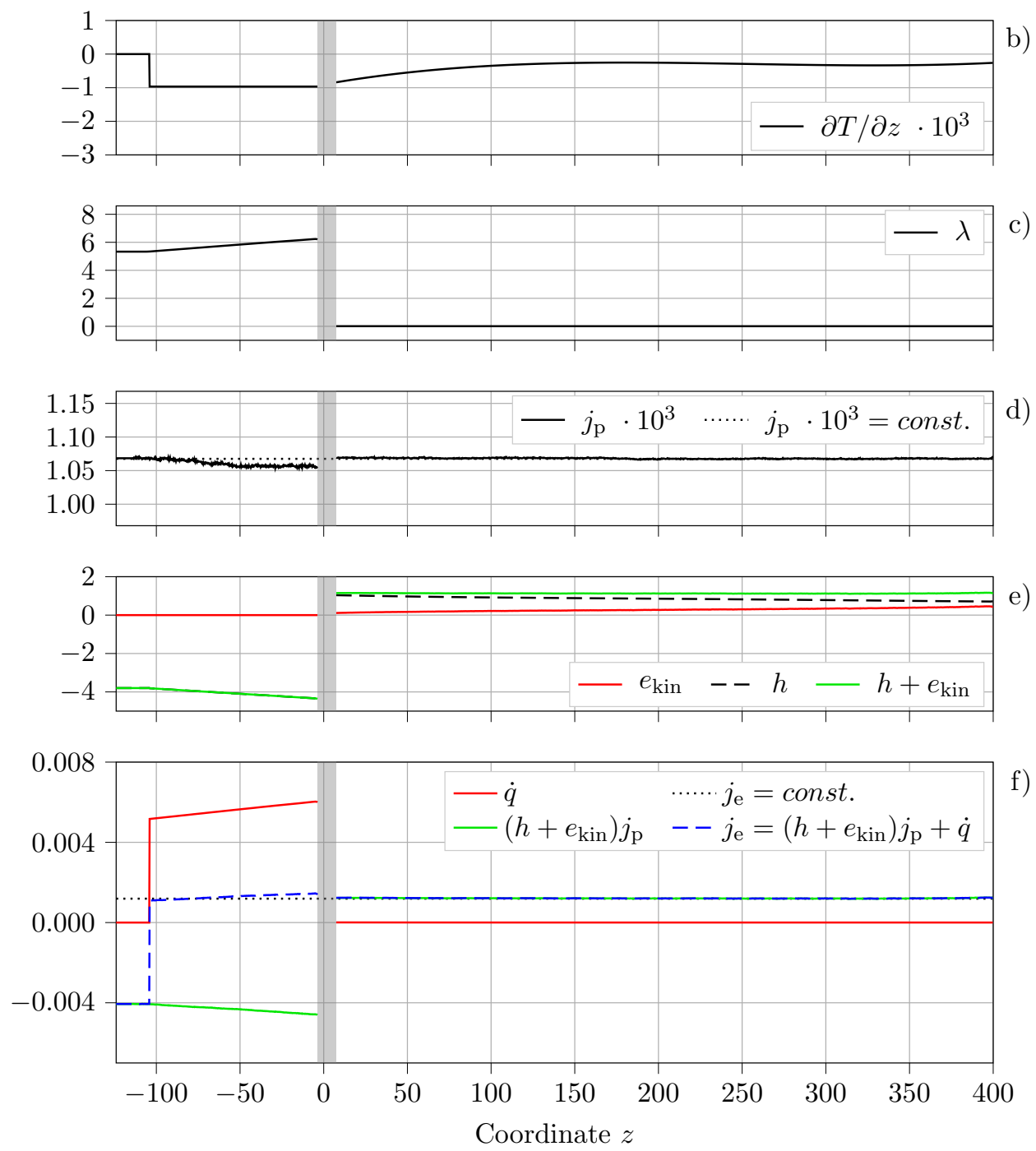

Figure 10. Evaluation of the energy flux $j_{\mathrm{e}}$ obtained by post-processing of profiles sampled during simulation with $T_{\text {liq }}=0.75$ and $L_{\mathrm{n}}=104$. a) Sampled profiles, i.e. density $\rho$, temperatures $T_{x y}$ and $T_{z}$ and their weighted average $T=\left(2 T_{x y}+T_{z}\right) / 3$ as well as hydrodynamic velocity $\left.v_{z} ; \mathrm{b}\right)$ First derivative of the temperature profile $\mathrm{d} T / \mathrm{d} z$; c) Thermal conductivity $\lambda$; d) Particle flux $j_{\mathrm{p}}=\rho v_{z}$; e) Enthalpy $h$, kinetic energy $e_{\text {kin }}$ and their sum $h+e_{\text {kin }}$; f) Energy flux $j_{\mathrm{e}}$ obtained from Eq. (11). Within the interface region marked by a grey rectangle around $z=0$, only sampled profiles of a) are shown, since the quantities depicted in b) to f) cannot straightforwardly be evaluated there. 


\section{Conclusions}

The stationary evaporation behavior of the LJTS2.5 fluid was under investigation. A series of $35 \mathrm{MD}$ simulations with bulk liquid temperatures $T_{\text {liq }}=$ $0.625,0.65,0.70,0.75,0.80$ and length of the non-thermostated liquid region between $L_{\mathrm{n}}=5.2$ and 208 was carried out. A vacuum boundary condition on the vapor side induced evaporation. To achieve stationarity of that process, the liquid was replenished continuously at $T_{\text {liq. }}$.

The interface temperature $T_{\mathrm{IP}}$ and the particle flux $j_{\mathrm{p}}$ were found to exponentially decrease with rising length of the non-thermostated liquid region $L_{\mathrm{n}}$. Both of these properties were correlated by functions of the bulk liquid temperature and the length of the non-thermostated liquid region, which allowed for an extrapolation to $L_{\mathrm{n}} \rightarrow \infty$ that is necessary for a comparison with macroscopic experimental data.

Following the Hertz-Knudsen approach, the evaporation coefficient $\alpha$ was determined by the ratio of the particle flux $j_{\mathrm{p}}$ and the Hertz flux $j^{\mathrm{H}}$. Since the particle flux depends heavily on the bulk liquid temperature $T_{\text {liq }}$ and the length $L_{\mathrm{n}}$, the evaporation coefficient does as well. These results are in good agreement with literature data based on the kinetic equation for fluids and MD.

One important message of Ref. [41] was that the particle flux $j_{\mathrm{p}}$ solely depends on the interface temperature $T_{\mathrm{IP}}$. This was confirmed by the entire set of the present simulations, covering a much larger range of conditions. The interface temperature was the same, regardless of whether a given simulation was conducted with a high bulk liquid temperature $T_{\text {liq }}$ and large length of the non-thermostated liquid region $L_{\mathrm{n}}$, or a lower bulk liquid temperature $T_{\text {liq }}$ in combination with a smaller length $L_{\mathrm{n}}$. The same holds for the back flux ratio.

A detailed analysis of the energy flux $j_{\mathrm{e}}$ and the heat flux $\dot{q}$ elucidated the energy transport in the studied evaporation process, in which the interface temperature $T_{\mathrm{IP}}$ was identified as a key quantity. On the liquid side, $T_{\mathrm{IP}}$ determines the temperature gradient between the bulk liquid and the interface and consequently controls the heat flux $\dot{q}$ that supplies the interface with energy to balance the enthalpy of evaporation that is continuously carried away. Therefore, the demand for energy that has to be satisfied by the heat flux depends essentially on the particle flux $j_{\mathrm{p}}$, which is also determined by $T_{\mathrm{IP}}$. Hence, $T_{\mathrm{IP}}$ connects the heat flux $\dot{q}$ on the liquid side and the particle flux $j_{\mathrm{p}}$ over the energy balance (11) such that for given boundary conditions, stationarity with $j_{\mathrm{e}}=$ const. was only attained at a certain interface temperature $T_{\mathrm{IP}}$.

\section{Acknowledgements}

The work was supported by the German Research Foundation (DFG) through SFBTRR 75, Project number 84292822 - "Droplet Dynamics under Extreme Ambient Conditions" and the Federal Ministry of Education and Research (BMBF) under the grant 01IH16008 "TaLPas: Task-basierte Lastverteilung und Auto-Tuning in der Partikelsimulation". The simulations were performed on the national supercomputer Cray XC40 (Hazel Hen) at the High Performance Computing Center Stuttgart (HLRS) as well as on the cluster Cray CS500 (Noctua) at the Paderborn Center for Parallel Computing $\left(\mathrm{PC}^{2}\right)$ and the supercomputer SuperMUC-NG at the Leibniz Supercomputing Centre Garching (LRZ). We thank S. Jöns for providing assistance with the integration of the employed LJTS2.5 equation of state into our post-processing procedure. 


\section{References}

[1] H. Hertz, Ann. Phys. 253 (10), 177-193 (1882).

[2] M. Knudsen, Ann. Phys. 352 (13), 697-708 (1915).

[3] F. Hensel and E.U. Franck, Rev. Mod Phys. 40 (4), 697-703 (1968).

[4] W. Prüger, Z. f. Physik 115 (3-4), 202-244 (1940).

[5] K. Leven, Wärme- und Kältetechn. 44, 161-167 (1942).

[6] K. Hammeke, Ph. D. thesis, Universität Münster, Germany, (1955).

[7] H.K. Cammenga, Habilitation thesis, Techn. Univ. Braunschweig, Germany, (1972).

[8] U. Narusawa and G.S. Springer, J. Colloid Interface Sci. 50 (2), 392-395 (1975).

[9] K. Hickman and W. Kayser, J. Colloid Interface Sci. 52 (3), 578-581 (1975).

[10] D. Schreiber and H.K. Cammenga, Ber. Bunsenges. Phys. Chem. 85 (10), 909-914 (1981).

[11] G.T. Barnes and D.S. Hunter, J. Colloid Interface Sci. 88 (2), 437-443 (1982).

[12] M. Faubel, S. Schlemmer and J.P. Toennies, Z. f. Physik D 10 (2-3), 269-277 (1988).

[13] C.A. Ward and D. Stanga, Phys. Rev. E 64 (5), 051509 (2001).

[14] S. Popov, A. Melling, F. Durst and C. Ward, Int. J. Heat Mass Transf. 48 (11), 2299-2309 (2005).

[15] V.K. Badam, V. Kumar, F. Durst and K. Danov, Exp. Therm. Fluid Sci. 32 (1), 276-292 (2007).

[16] K.H. Le, A. Kharaghani, C. Kirsch and E. Tsotsas, Drying Techn. 35 (13), 1584-1601 (2017).

[17] R. Hołyst, M. Litniewski, D. Jakubczyk, M. Zientara and M. Woźniak, Soft Matt. 9 (32), 7766-7774 (2013).

[18] R. Hołyst, M. Litniewski and D. Jakubczyk, Soft Matt. 13 (35), 5858-5864 (2017).

[19] F. Bošnjaković, Techn. Mech. u. Thermodyn. 1 (10), 358-362 (1930).

[20] A. Frezzotti, L. Gibelli and S. Lorenzani, Phys. Fluids 17 (1), 012102 (2005).

[21] Ø. Wilhelmsen, T.T. Trinh, A. Lervik, V.K. Badam, S. Kjelstrup and D. Bedeaux, Phys. Rev. E 93 (3), 032801 (2016).

[22] A. Frezzotti, L. Gibelli, D.A. Lockerby and J.E. Sprittles, Phys. Rev. Fluids 3 (5), 054001 (2018).

[23] R. Stierle, C. Waibel, J. Gross, C. Steinhausen, B. Weigand and G. Lamanna, Int. J. Heat Mass Transf. 151, 119450 (2020).

[24] G.T. Barnes and H.K. Cammenga, J. Colloid Interface Sci. 72 (1), 140-141 (1979).

[25] G. Nagayama and T. Tsuruta, J. Chem. Phys. 118 (3), 1392-1399 (2003).

[26] A. Lotfi, Molekulardynamische Simulationen an Fluiden: Phasengleichgewicht und Verdampfung, Fortschritt-Berichte VDI, Vol. 3/335 (VDI-Verlag, Düsseldorf, 1993).

[27] L.N. Long, M.M. Micci and B.C. Wong, Comput. Phys. Commn. 96 (2-3), 167-172 (1996).

[28] S.I. Anisimov, D.O. Dunikov, V.V. Zhakhovskii and S.P. Malyshenko, J. Chem. Phys. 110 (17), 8722-8729 (1999).

[29] L. Consolini, S.K. Aggarwal and S. Murad, Int. J. Heat Mass Transf. 46 (17), 3179-3188 (2003).

[30] K. Yasuoka, M. Matsumoto and Y. Kataoka, J. Chem. Phys. 101 (9), 7904-7911 (1994).

[31] T. Tsuruta, N. Sakamoto and T. Masuoka, Thermal. Sci. Eng. 3, 85-90 (1995).

[32] T. Tsuruta, H. Tanaka and T. Masuoka, Int. J. Heat Mass Transf. 42 (22), 4107-4116 (1999).

[33] T. Ishiyama, T. Yano and S. Fujikawa, Phys. Fluids 16 (8), 2899-2906 (2004).

[34] S. Sumardiono and J. Fischer, Microfluid. Nanofluid. 3 (2), 127-140 (2007).

[35] R. Hołyst and M. Litniewski, Phys. Rev. Lett. 100 (5), 055701 (2008).

[36] R. Holyst and M. Litniewski, J. Chem. Phys. 130 (7), 074707 (2009).

[37] A. Frezzotti, in AIP Conf. Proc. (AIP, Geneva, 2011), pp. 161-166.

[38] J. Yu and H. Wang, Int. J. Heat Mass Transf. 55 (4), 1218-1225 (2012). 
[39] T. Ishiyama, S. Fujikawa, T. Kurz and W. Lauterborn, Phys. Rev. E 88 (4), 042406 (2013).

[40] A. Lotfi, J. Vrabec and J. Fischer, Int. J. Heat Mass Transf. 73, 303-317 (2014).

[41] M. Heinen, J. Vrabec and J. Fischer, J. Chem. Phys. 145 (8), 081101 (2016).

[42] Y. Tang, G.S. Grest and S. Cheng, Langmuir 34 (24), 7161-7170 (2018).

[43] M. Heinen and J. Vrabec, J. Chem. Phys. 151 (4), 044704 (2019).

[44] V.V. Zhakhovsky, A.P. Kryukov, V.Y. Levashov, I.N. Shishkova and S.I. Anisimov, PNAS 116 (37), 18209-18217 (2019).

[45] R.S. Chatwell, M. Heinen and J. Vrabec, Int. J. Heat Mass Transf. 132, 1296-1305 (2019).

[46] R. Hołyst, M. Litniewski and D. Jakubczyk, Soft Matt. 11 (36), 7201-7206 (2015).

[47] S. Stephan, D. Schaefer, K. Langenbach and H. Hasse, Mol. Phys. 0, e1810798 (2020).

[48] H.K. Cammenga, Current topics in materials science V, 335-446 (1980).

[49] A. Frezzotti, Phys. Fluids 23 (3), 030609 (2011).

[50] A.H. Persad and C.A. Ward, Chem. Rev. 116 (14), 7727-7767 (2016).

[51] S.S. Sazhin, Fuel 196, 69-101 (2017).

[52] M. Volmer, Kinetik der Phasenbildung (Steinkopff-Verlag, Dresden/Leipzig, 1939).

[53] R.W. Schrage, A Theoretical Study of Interface Mass Transfer (Columbia University Press, New York, 1953).

[54] S. Fujikawa, T. Yano and M. Watanabe, Vapor-Liquid Interfaces, Bubbles and Droplets (Springer, Berlin/Heidelberg, 2011).

[55] S. Sazhin, Droplets and Sprays (Springer, London, 2014).

[56] Y. Koizumi, M. Shoji, M. Monde, Y. Takata and N. Nagai, editors, Boiling (Elsevier, Amsterdam, 2017).

[57] S. Basu, A.K. Agarwal, A. Mukhopadhyay and C. Patel, editors, Droplets and sprays. Applications for combustion and propulsion (Springer, Singapore, 2018).

[58] Y.B. Zudin, Non-equilibrium Evaporation and Condensation Processes: Analytical Solutions (Springer International Publishing, Switzerland, 2018).

[59] D.N. Gerasimov and E.I. Yurin, Kinetics of Evaporation (Springer International Publishing, Switzerland, 2018).

[60] I. Hölzel, O. Knacke and H. Parthey, Landolt-Börnstein, Vol. 2.5b, 6th ed. (Springer, Berlin/Heidelberg, 1968).

[61] G.M. Pound, J. Phys. Chem. Ref. Data 1 (1), 135-146 (1972).

[62] I.W. Eames, N.J. Marr and H. Sabir, Int. J. Heat Mass Transf. 40 (12), 2963-2973 (1997).

[63] R. Marek and J. Straub, Int. J. Heat Mass Transf. 44 (1), 39-53 (2001).

[64] M. Grmela, J. Stat. Phys. 3 (3), 347-364 (1971).

[65] J. Karkheck and G. Stell, J. Chem. Phys. 75 (3), 1475-1487 (1981).

[66] J. Vrabec, G.K. Kedia, G. Fuchs and H. Hasse, Mol. Phys. 104 (9), 1509-1527 (2006).

[67] P.G. Bolhuis and D. Chandler, J. Chem. Phys. 113 (18), 8154-8160 (2000).

[68] S. Toxvaerd, J. Phys. Chem. C 111 (43), 15620-15624 (2007).

[69] M. Thol, G. Rutkai, R. Span, J. Vrabec and R. Lustig, Int. J. Thermophys. 36 (1), 25-43 (2015).

[70] C. Niethammer, S. Becker, M. Bernreuther, M. Buchholz, W. Eckhardt, A. Heinecke, S. Werth, H.J. Bungartz, C.W. Glass, H. Hasse, J. Vrabec and M. Horsch, J. Chem. Theory Comput. 10 (10), 4455-4464 (2014).

[71] G.H. Findenegg and T. Hellweg, Statistische Thermodynamik (Springer, Berlin/Heidelberg, 2015).

[72] J. Muscatello, E. Chacón, P. Tarazona and F. Bresme, Phys. Rev. Lett. 119 (4) (2017).

[73] R. Delgado-Buscalioni and P.V. Coveney, J. Chem. Phys. 119 (2), 978-987 (2003).

[74] Ø. Wilhelmsen, T.T. Trinh and A. Lervik, Phys. Rev. E 97 (1) (2018).

[75] S.I. Anisimov, Zh. Eksp. Teor. Fiz. 54, 399 (1968).

[76] J. Fischer, Phys. Fluids 19 (9), 1305-1311 (1976).

[77] E. Bird and Z. Liang, Phys. Rev. E 100 (4), 043108 (2019).

[78] R. Lustig, Fluid Phase Equilib. 32 (2), 117-137 (1987).

[79] A. Friedrich and R. Lustig, J. Mol. Liq. 98-99, 243-261 (2002). 
[80] M. Fioroni and D. Vogt, J. Phys. Chem. B 108 (31), 11774-11781 (2004).

[81] L. von Bogdandy, H.G. Kleist and O. Knacke, Z. Elektrochemie 59, 460-462 (1955).

[82] M.K. Baranaev, Žur. Fiz. Chim. 13, 1635 (1939).

[83] K. Hickman, Desalination 1 (1), 13-29 (1966).

[84] H. Schlichting and K. Gersten, Boundary-Layer Theory (Springer, Berlin/Heidelberg, 2017).

[85] M.P. Lautenschlaeger and H. Hasse, Fluid Phase Equilib. 482, 38-47 (2019).

[86] E.W. Lemmon and R.T. Jacobsen, Int. J. Thermophys. 25 (1), 21-69 (2004).

[87] M. Heier, S. Stephan, J. Liu, W.G. Chapman, H. Hasse and K. Langenbach, Mol. Phys. 116 (15-16), 2083-2094 (2018).

[88] T. Hitz, S. Joens, M. Heinen, J. Vrabec and C.D. Munz, submitted to J. Comput. Phys., see also https://arxiv.org/abs/2005.06910 (2020). 


\section{Supplementary Material to:}

\section{Evaporation driven by conductive heat transport}

Simon Homes ${ }^{\mathrm{a}}$, Matthias Heinen ${ }^{\mathrm{a}},{\text { Jadran } \text { Vrabec }^{\mathrm{a}, *} \text { and Johann Fischer }}^{\mathrm{b}}$

${ }^{a}$ Technische Universität Berlin, Thermodynamik und Thermische Verfahrenstechnik, Ernst-Reuter-Platz 1, 10587 Berlin, Germany.

${ }^{\mathrm{b} U n i v e r s i t a ̈ t ~ f u ̈ r ~ B o d e n k u l t u r, ~ I n s t i t u t ~ f u ̈ r ~ V e r f a h r e n s-~ u n d ~ E n e r g i e t e c h n i k, ~ M u t h g a s s e ~ 107, ~}$ 1190 Wien, Austria.

The following supplementary material includes the nomenclature as well as several additional figures. 


\section{Nomenclature}

Table 1 lists the used symbols, super- and subscripts together with a description.

\begin{tabular}{ll}
\hline Symbol & Discription \\
\hline$A$ & Parameter \\
$b$ & Parameter \\
$B$ & Parameter \\
$c$ & Parameter \\
$C$ & Parameter \\
$e$ & Energy \\
$f$ & Parameter \\
$g$ & Parameter \\
$h$ & Enthalpy \\
$j$ & Flux \\
$L$ & Length \\
$m$ & Mass \\
$n$ & Time step \\
$T$ & Temperature \\
$v$ & Velocity \\
$x, y, z$ & Cartesian coordinates \\
\hline$\alpha$ & Evaporation coefficient \\
$\varepsilon$ & Energy parameter of potential model \\
$\lambda$ & Thermal conductivity \\
$\rho$ & Density \\
$\sigma$ & Size parameter of potential model \\
\hline
\end{tabular}

\begin{tabular}{ll}
\hline Superscript & Discription \\
\hline H & Hertz \\
sim & Simulation \\
$\infty$ & Infinity \\
+ & In positive direction \\
- & In negative direction \\
$\prime \prime$ & Saturated liquid \\
\hline & Saturated vapor \\
\hline Subscript & Discription \\
\hline e & Critical \\
FE & Energy \\
IP & Force extreme \\
liq & Inflection point \\
n & Liquid \\
p & Non-thermostated \\
sat & Particle \\
t & Saturation \\
tr & Thermostated \\
vac & Triple point \\
vap & Vacuum \\
\hline
\end{tabular}




\section{Figures}

Figs. 11 and 12 depict the coefficients of the respective fit over the bulk liquid temperature $T_{\text {liq. }}$. Figs. 13 to 47 show the evaluation of the energy flux $j_{\mathrm{e}}$ for each simulation. Panel description: a) Sampled profiles, i.e. density $\rho$, temperature $T_{x y}$ and $T_{z}$ and their weighted average $T=\left(2 T_{x y}+T_{z}\right) / 3$ as well as hydrodynamic velocity $\left.v_{z} ; \mathrm{b}\right)$ First derivative of the temperature profile $\mathrm{d} T / \mathrm{d} z ; \mathrm{c}$ ) Thermal conductivity $\lambda ; \mathrm{d}$ ) Particle flux $j_{\mathrm{p}}=\rho v_{z}$; e) Enthalpy $h$, kinetic energy $e_{\text {kin }}$ and their sum $h+e_{\text {kin }} ;$ f) Energy flux $j_{\mathrm{e}}$ obtained from Eq. (11) in the accompanied paper. Within the interface region marked by a grey rectangle around $z=0$, only sampled profiles of a) are shown, since the quantities depicted in b) to f) cannot straightforwardly be evaluated there. 

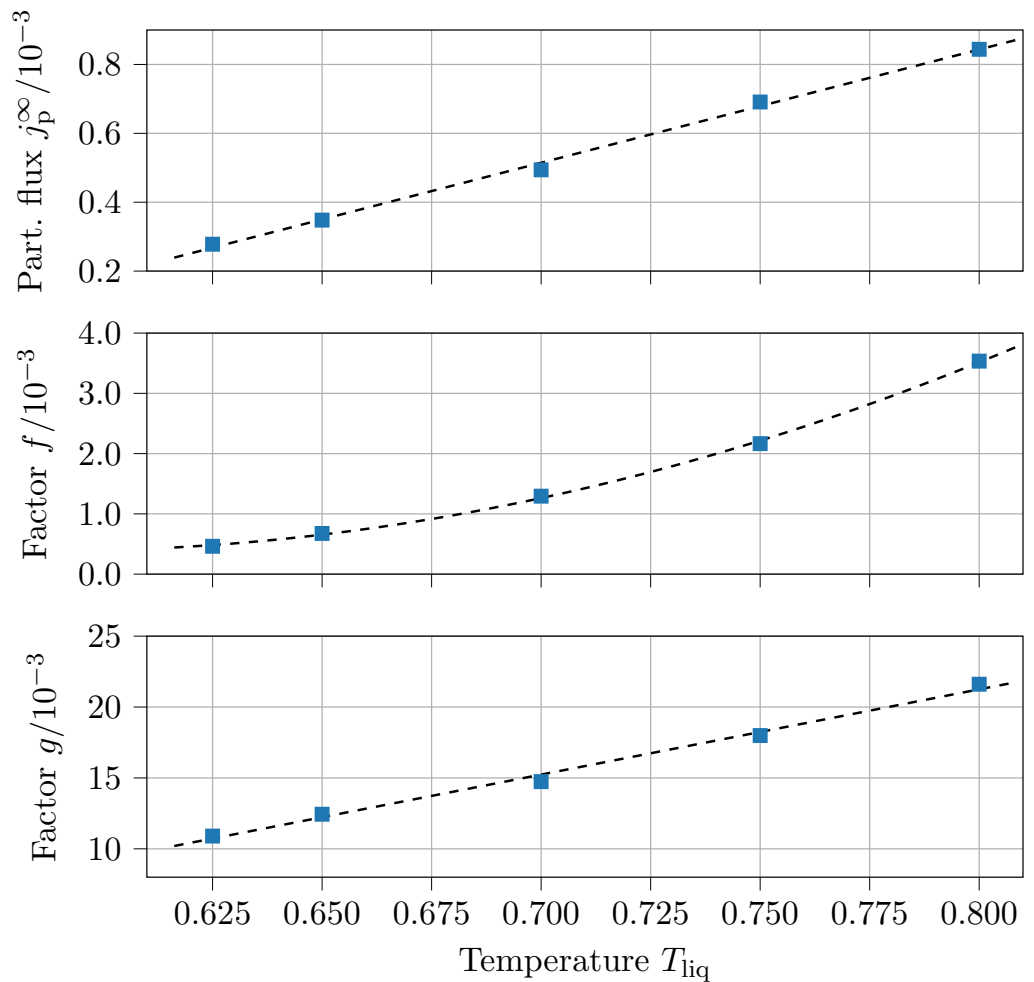

Figure 11. Coefficients of the fit (3) over the bulk liquid temperature $T_{\text {liq. }}$. The functions in Eqs. (4) are depicted as dashed lines.
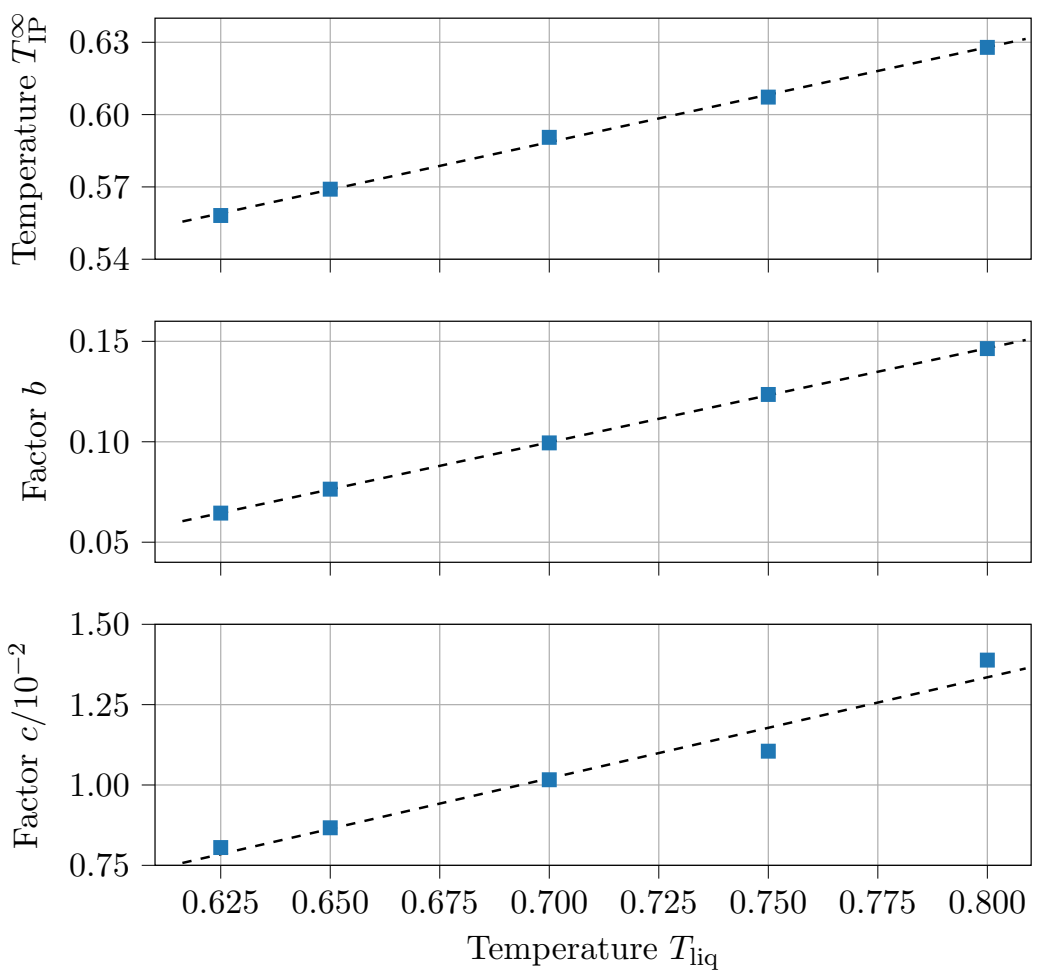

Figure 12. Coefficients of the fit (8) over the bulk liquid temperature $T_{\text {liq }}$. The linear functions in Eqs. (9) are depicted as dashed lines. 

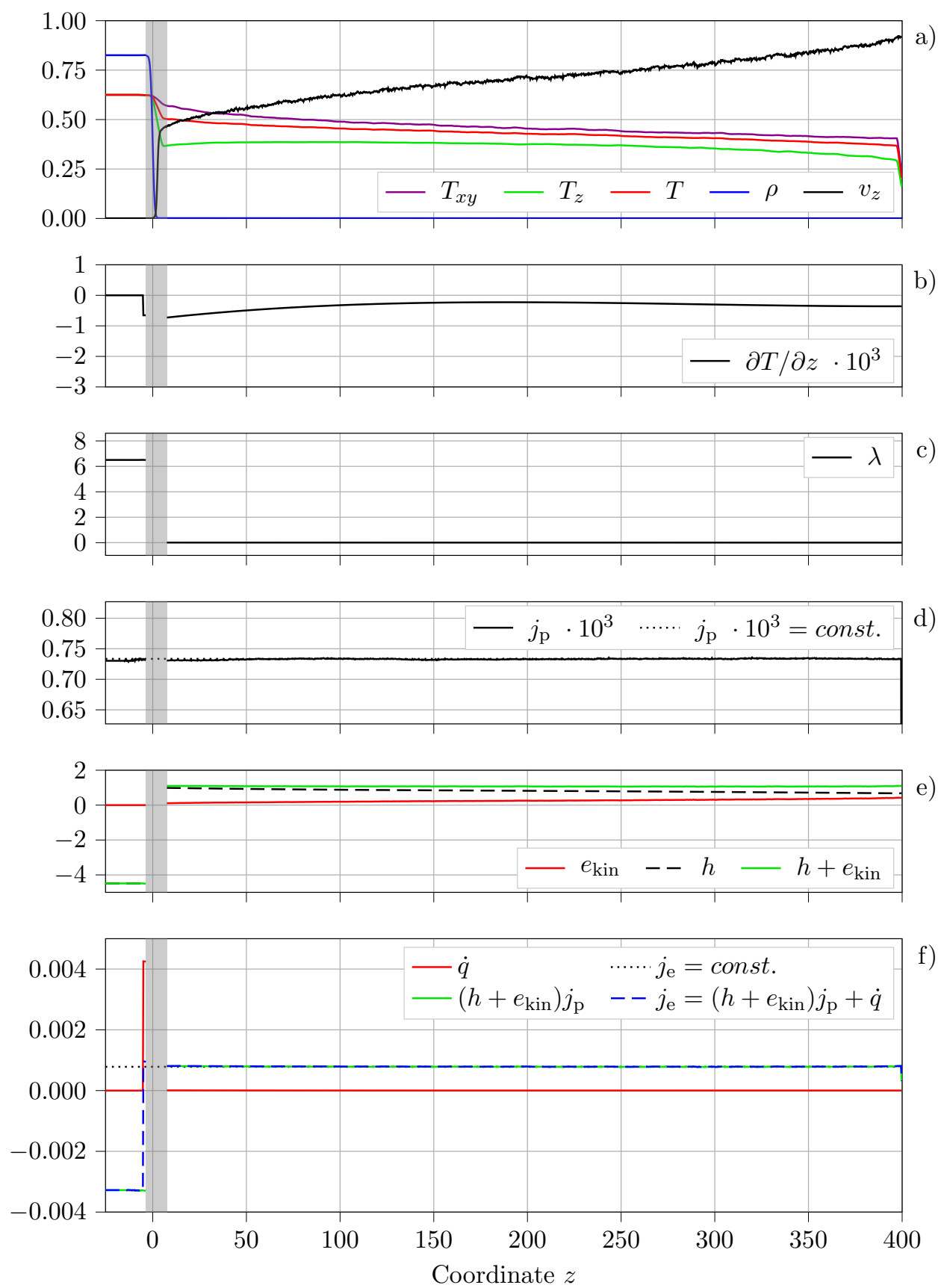

Figure 13. Evaluation of the energy flux $j_{\mathrm{e}}$ obtained by post-processing of profiles sampled during simulation with $T_{\text {liq }}=0.625$ and $L_{\mathrm{n}}=5.2$. Panels according to the description outlined above. 

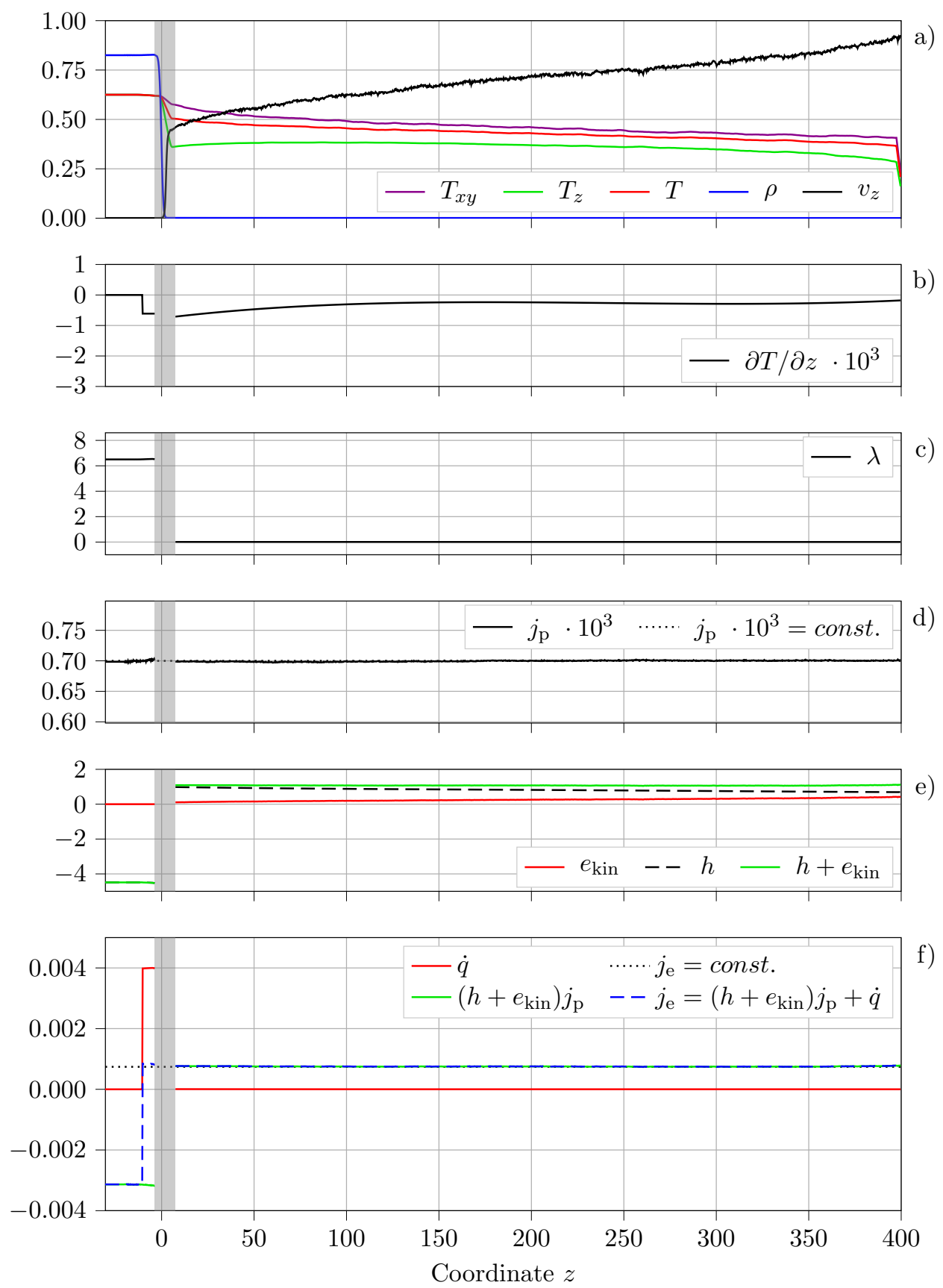

Figure 14. Evaluation of the energy flux $j_{\mathrm{e}}$ obtained by post-processing of profiles sampled during simulation with $T_{\text {liq }}=0.625$ and $L_{\mathrm{n}}=10.4$. Panels according to the description outlined above. 

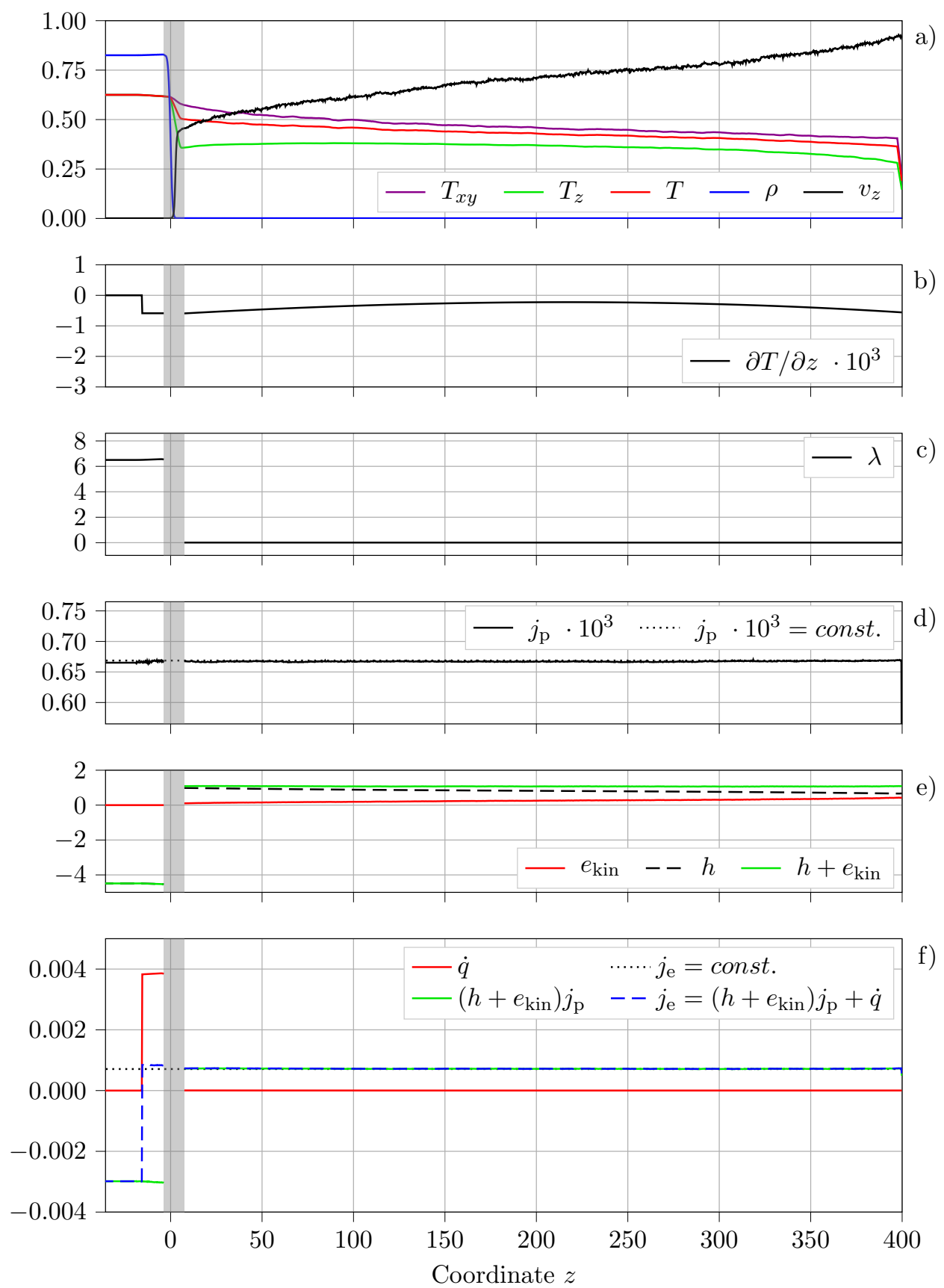

Figure 15. Evaluation of the energy flux $j_{\mathrm{e}}$ obtained by post-processing of profiles sampled during simulation with $T_{\text {liq }}=0.625$ and $L_{\mathrm{n}}=15.6$. Panels according to the description outlined above. 

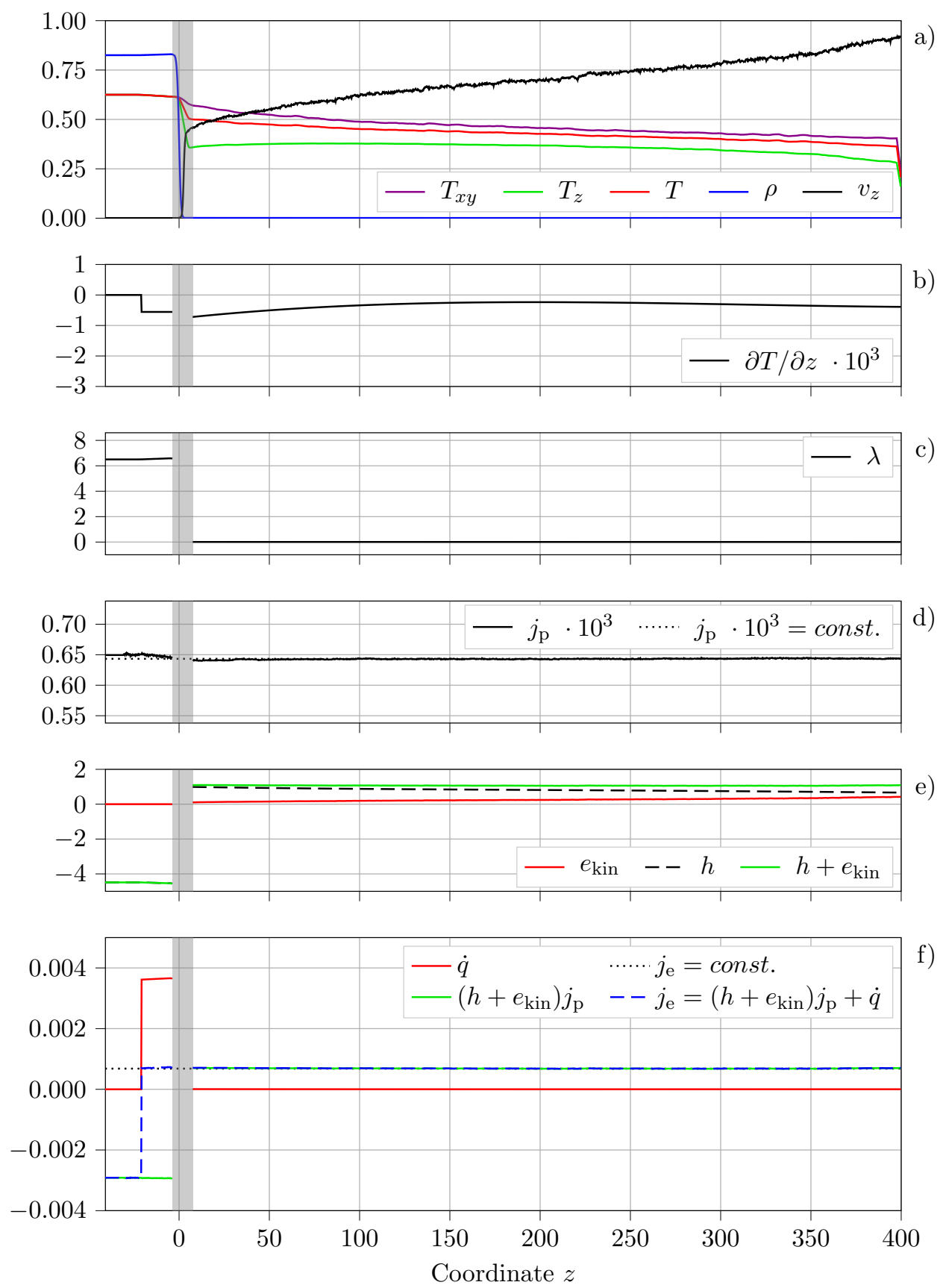

Figure 16. Evaluation of the energy flux $j_{\mathrm{e}}$ obtained by post-processing of profiles sampled during simulation with $T_{\text {liq }}=0.625$ and $L_{\mathrm{n}}=20.8$. Panels according to the description outlined above. 

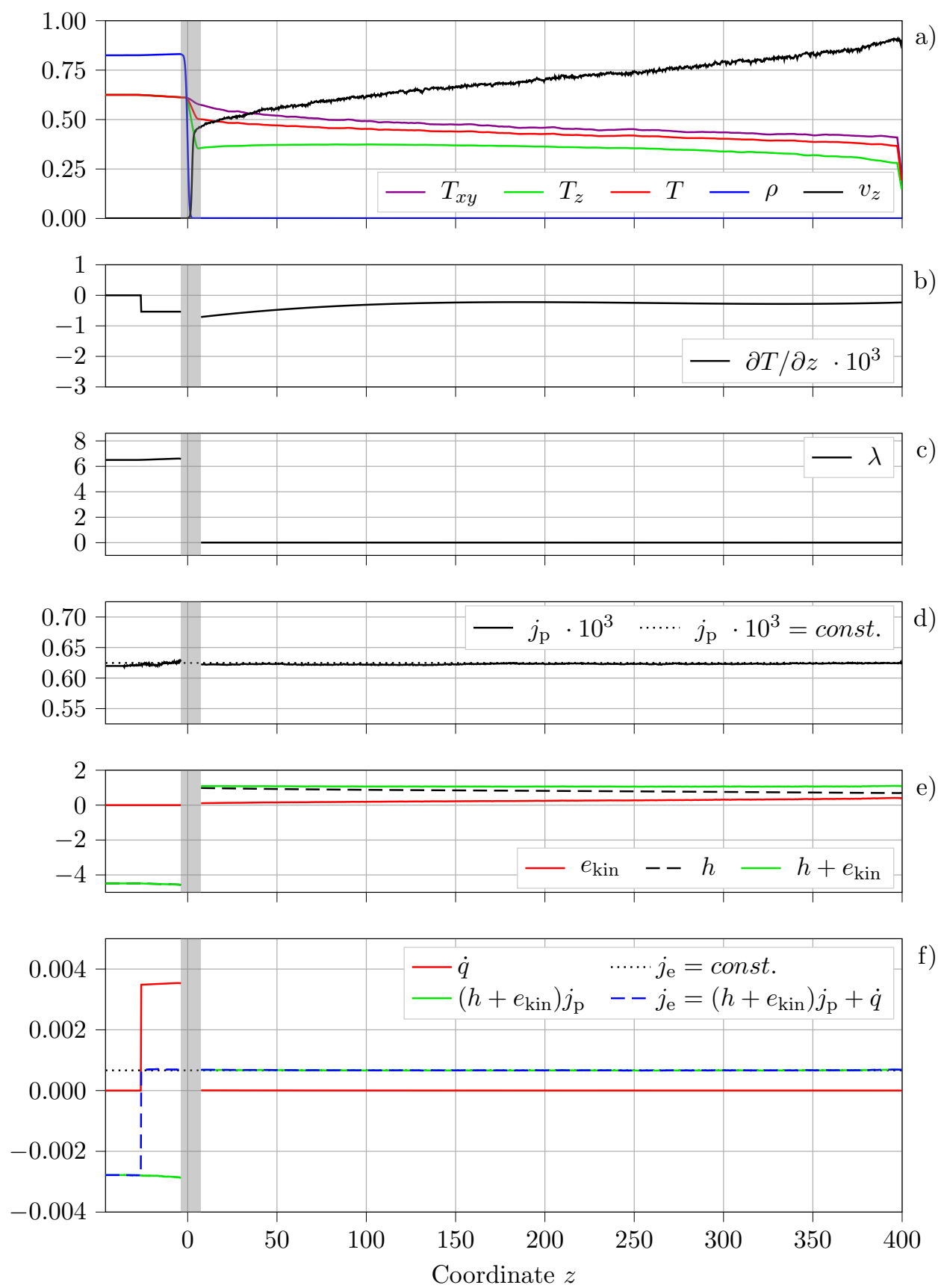

Figure 17. Evaluation of the energy flux $j_{\mathrm{e}}$ obtained by post-processing of profiles sampled during simulation with $T_{\text {liq }}=0.625$ and $L_{\mathrm{n}}=26$. Panels according to the description outlined above. 

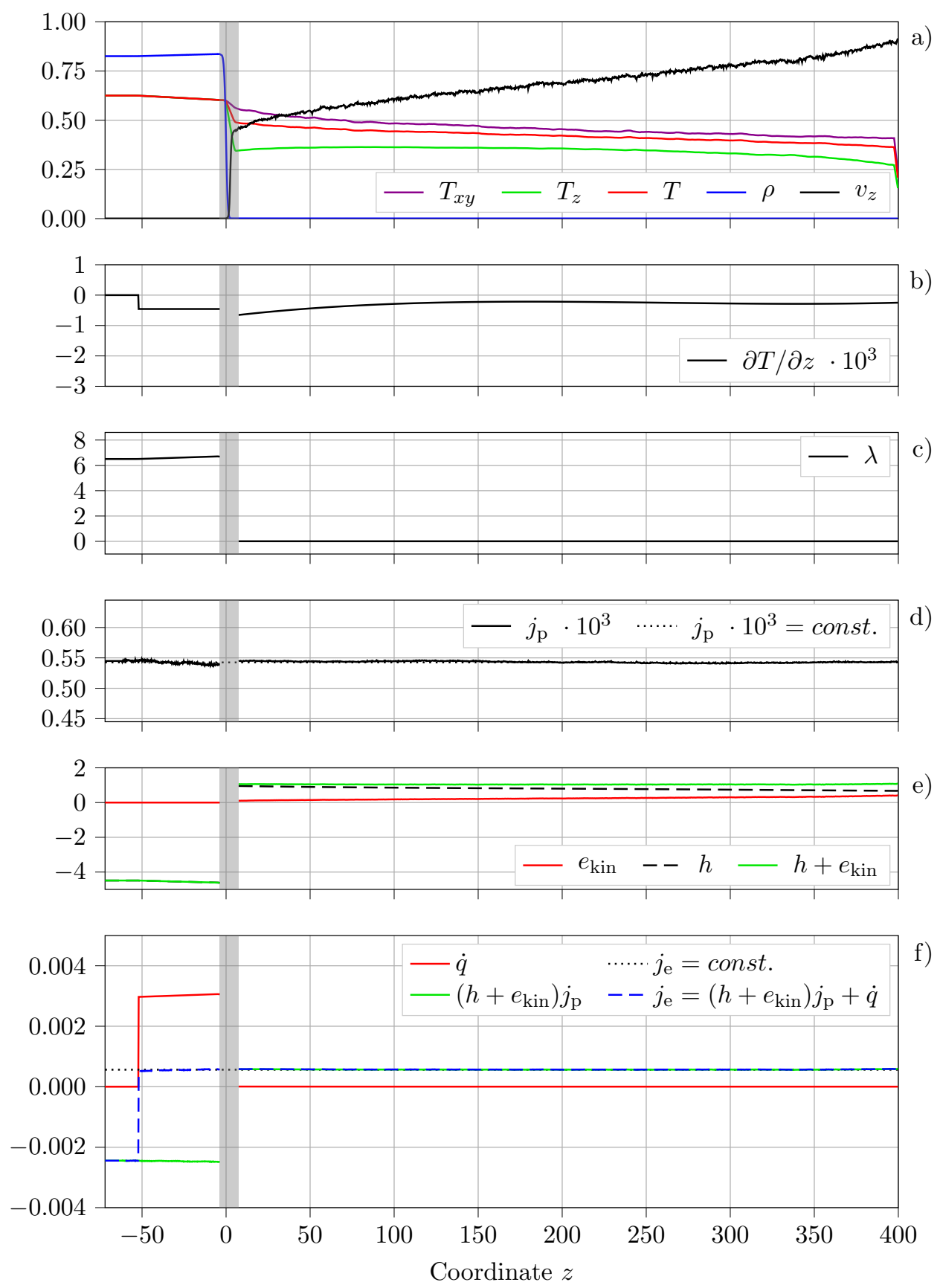

Figure 18. Evaluation of the energy flux $j_{\mathrm{e}}$ obtained by post-processing of profiles sampled during simulation with $T_{\text {liq }}=0.625$ and $L_{\mathrm{n}}=52$. Panels according to the description outlined above. 

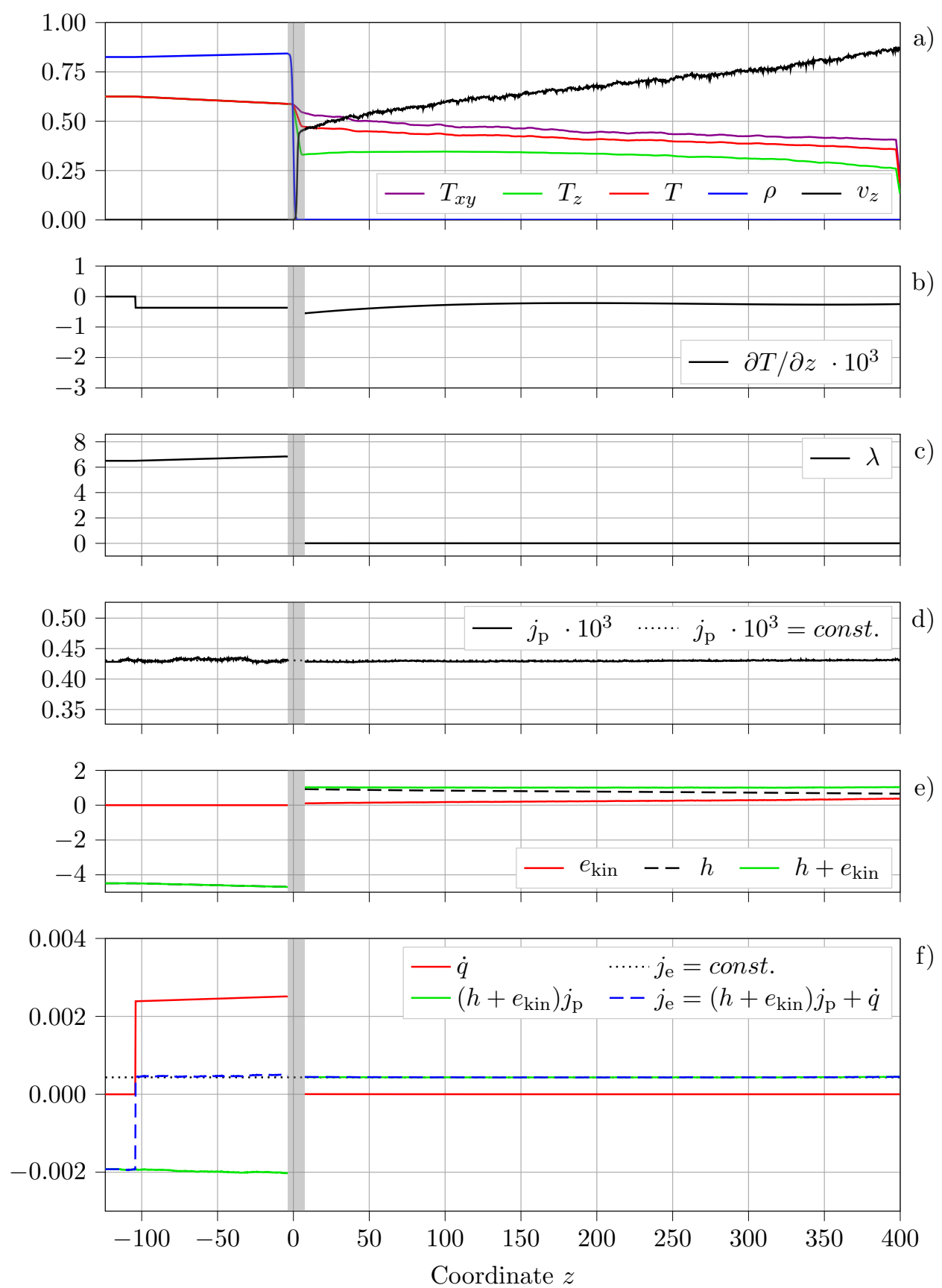

Figure 19. Evaluation of the energy flux $j_{\mathrm{e}}$ obtained by post-processing of profiles sampled during simulation with $T_{\text {liq }}=0.625$ and $L_{\mathrm{n}}=104$. Panels according to the description outlined above. 

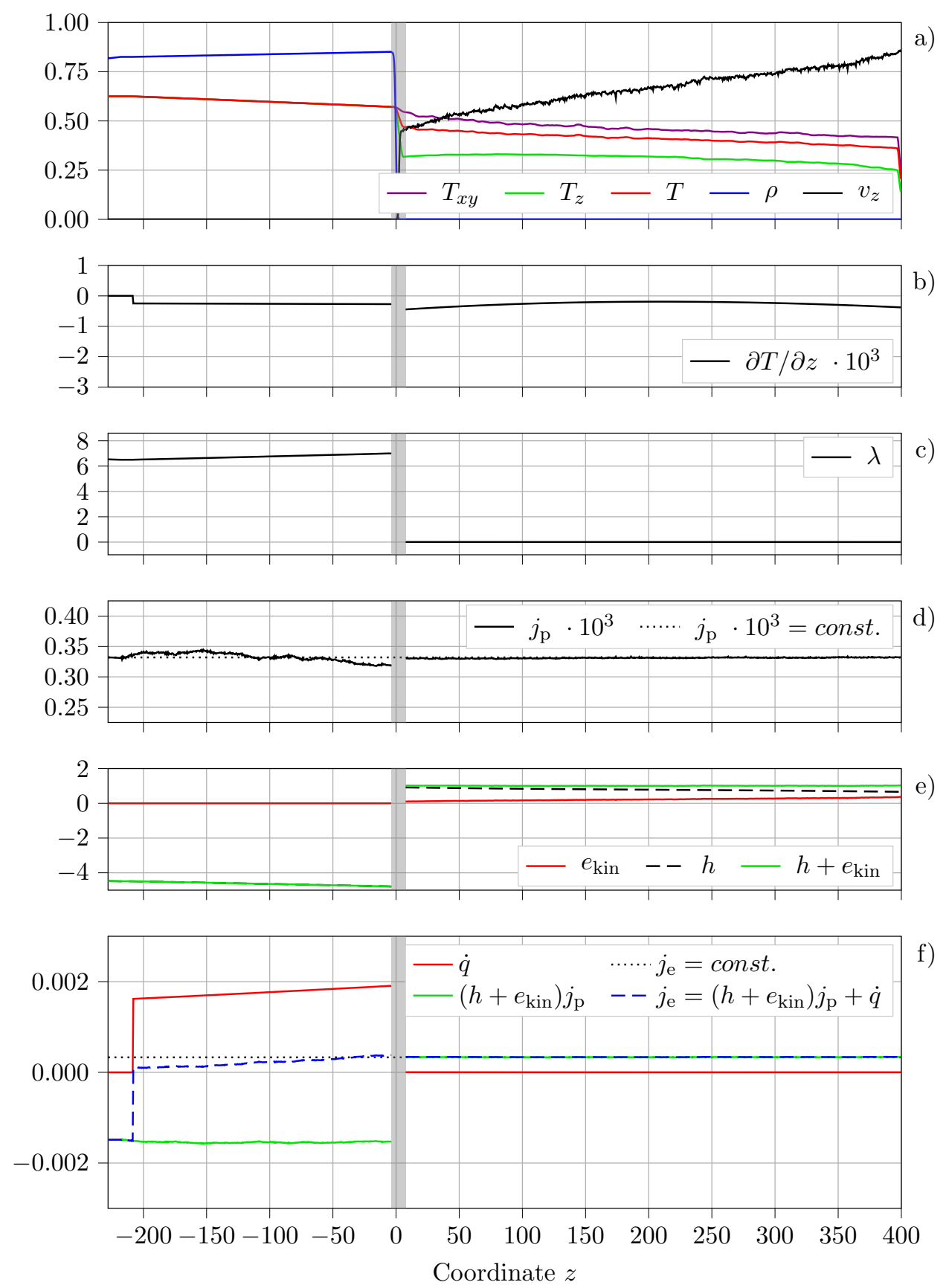

Figure 20. Evaluation of the energy flux $j_{\mathrm{e}}$ obtained by post-processing of profiles sampled during simulation with $T_{\text {liq }}=0.625$ and $L_{\mathrm{n}}=208$. Panels according to the description outlined above. 

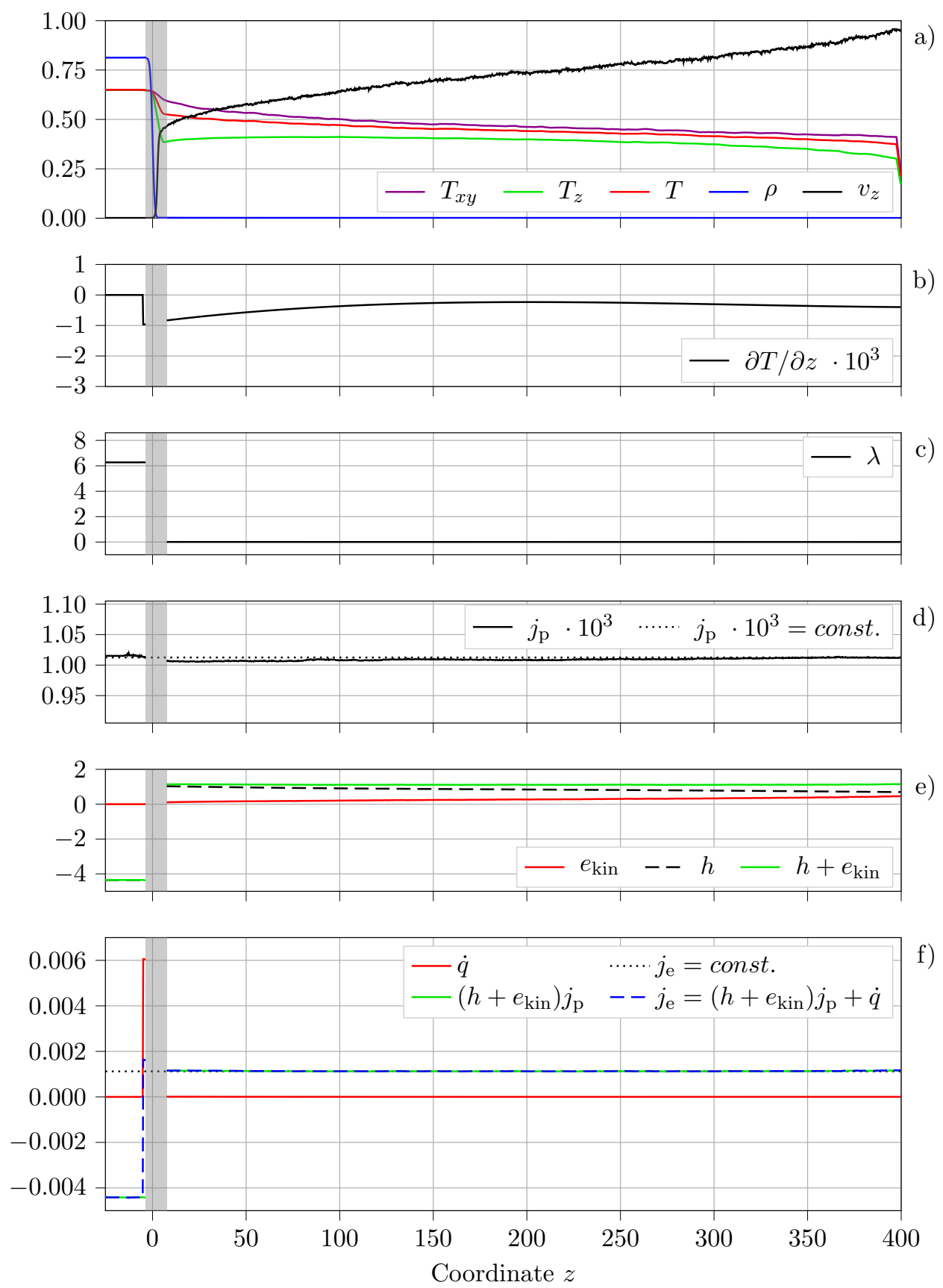

Figure 21. Evaluation of the energy flux $j_{\mathrm{e}}$ obtained by post-processing of profiles sampled during simulation with $T_{\text {liq }}=0.65$ and $L_{\mathrm{n}}=5.2$. Panels according to the description outlined above. 

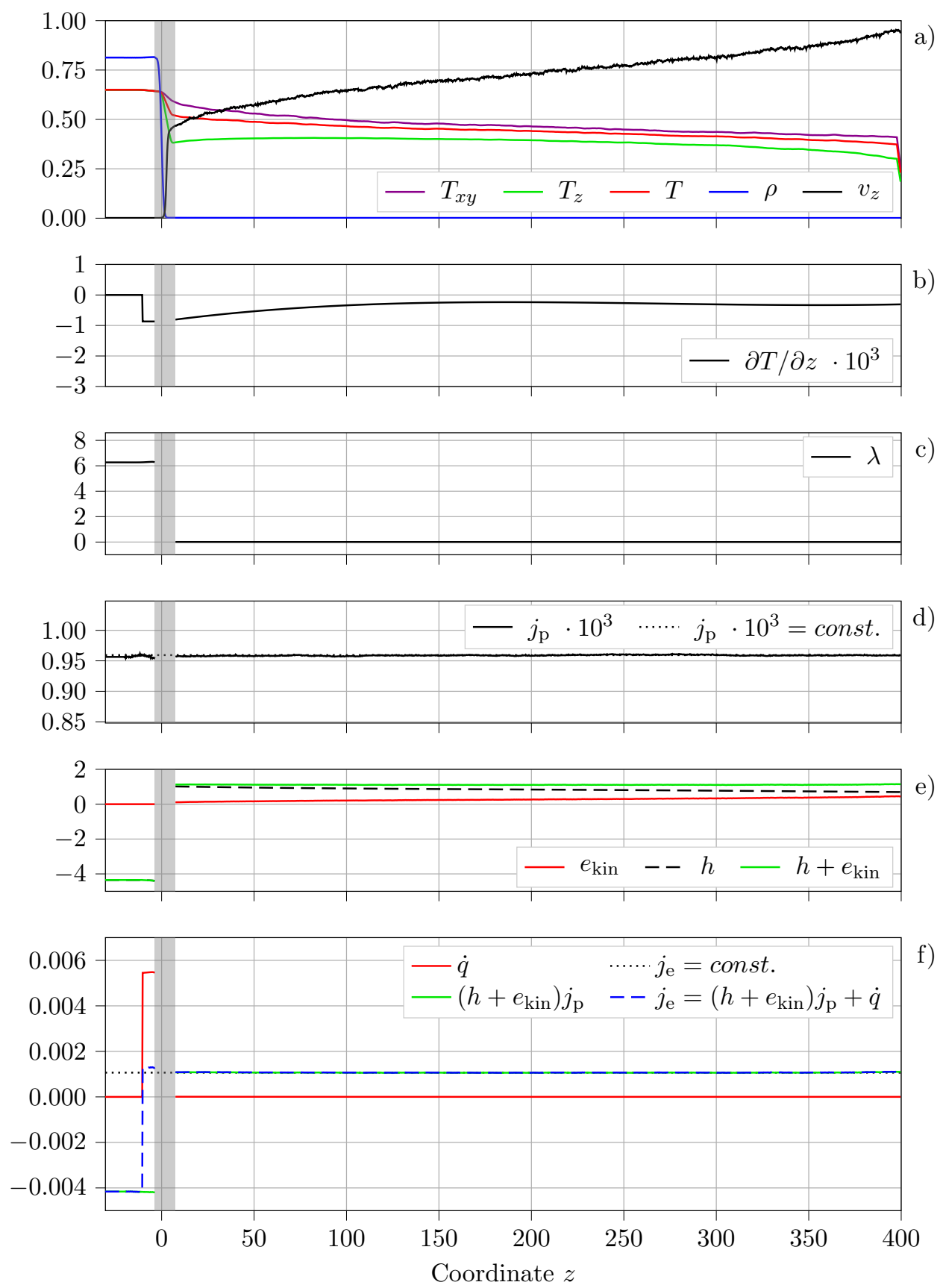

Figure 22. Evaluation of the energy flux $j_{\mathrm{e}}$ obtained by post-processing of profiles sampled during simulation with $T_{\text {liq }}=0.65$ and $L_{\mathrm{n}}=10.4$. Panels according to the description outlined above. 

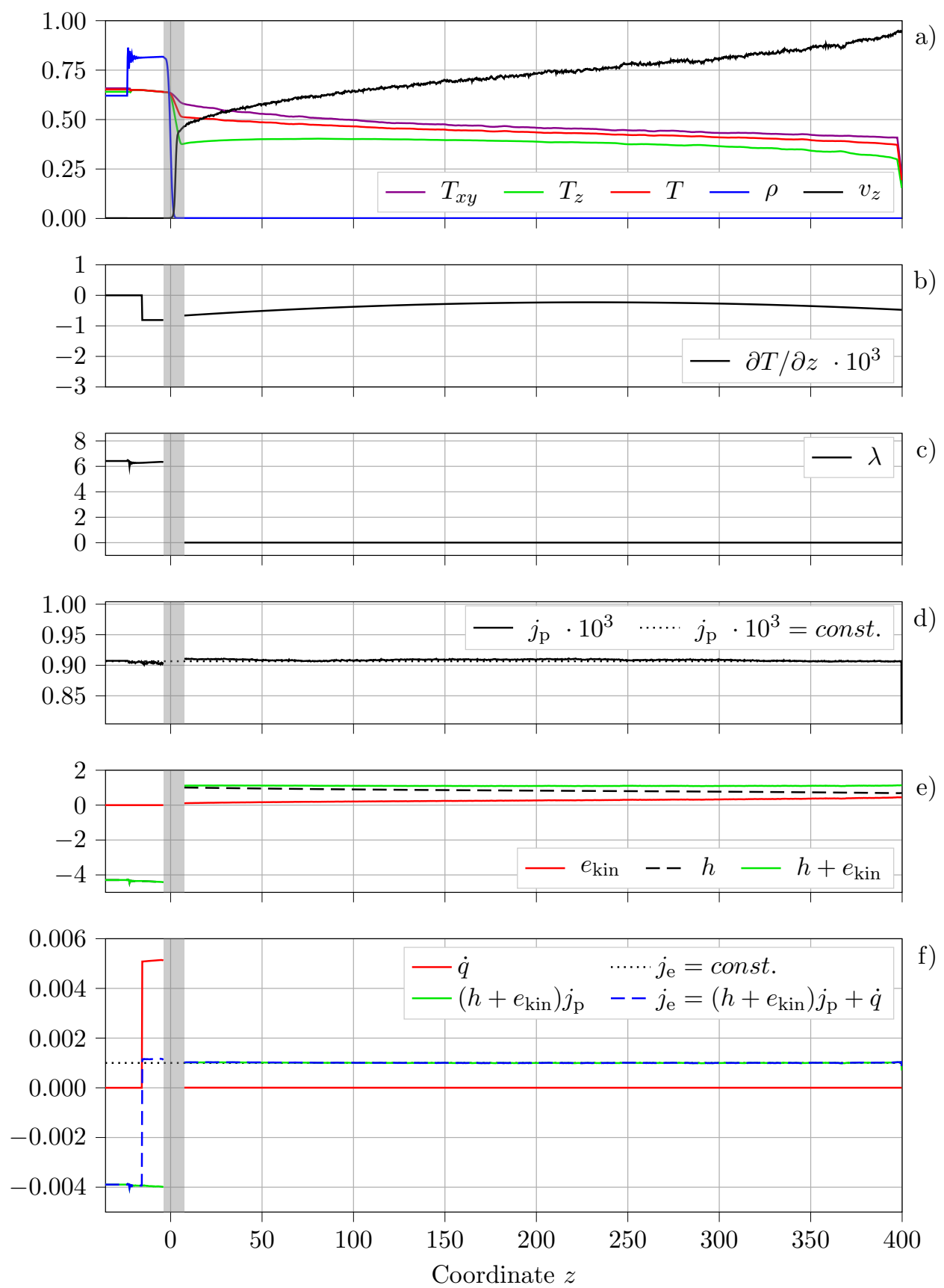

Figure 23. Evaluation of the energy flux $j_{\mathrm{e}}$ obtained by post-processing of profiles sampled during simulation with $T_{\text {liq }}=0.65$ and $L_{\mathrm{n}}=15.6$. Panels according to the description outlined above. 

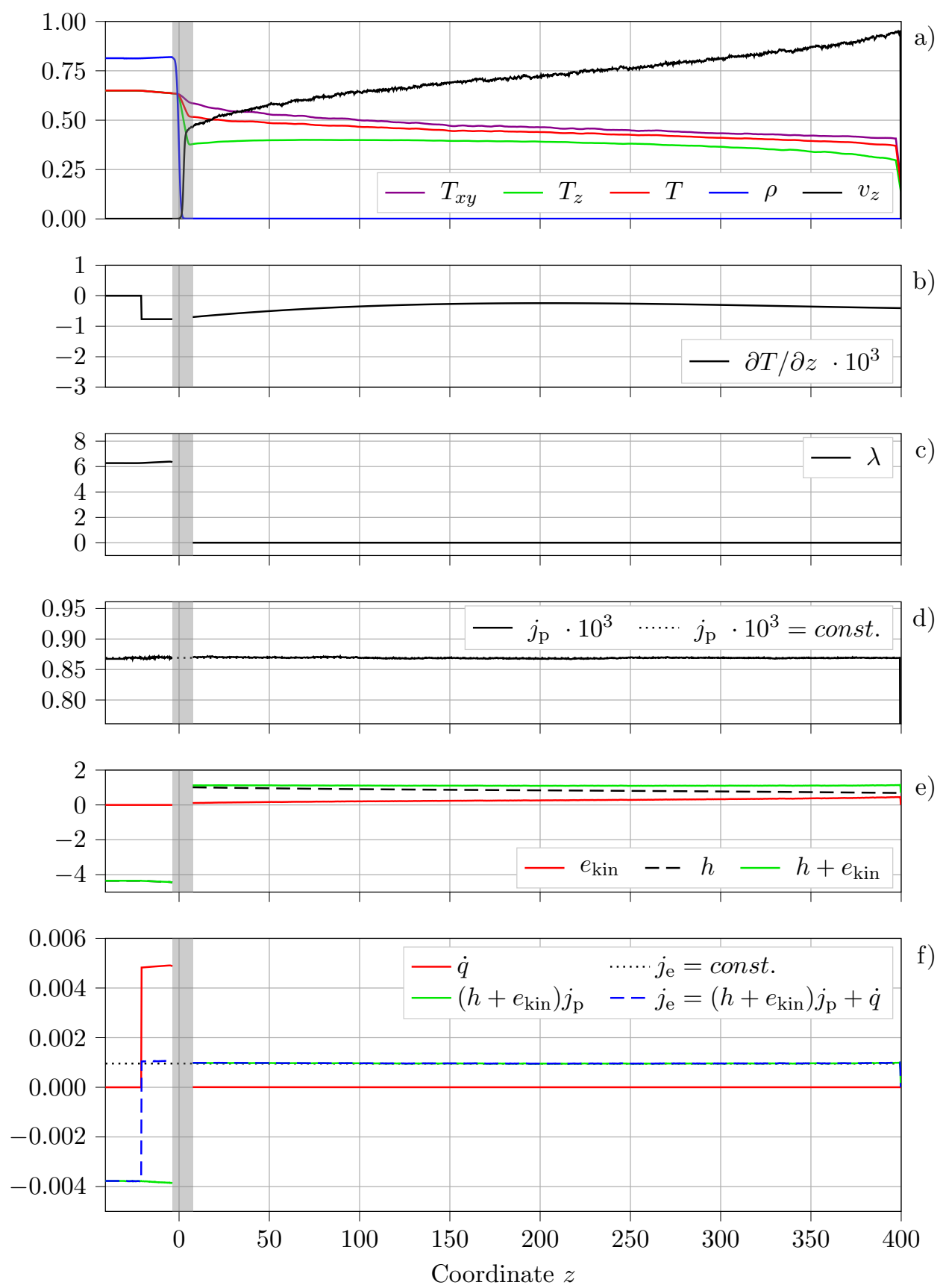

Figure 24. Evaluation of the energy flux $j_{\mathrm{e}}$ obtained by post-processing of profiles sampled during simulation with $T_{\text {liq }}=0.65$ and $L_{\mathrm{n}}=20.8$. Panels according to the description outlined above. 

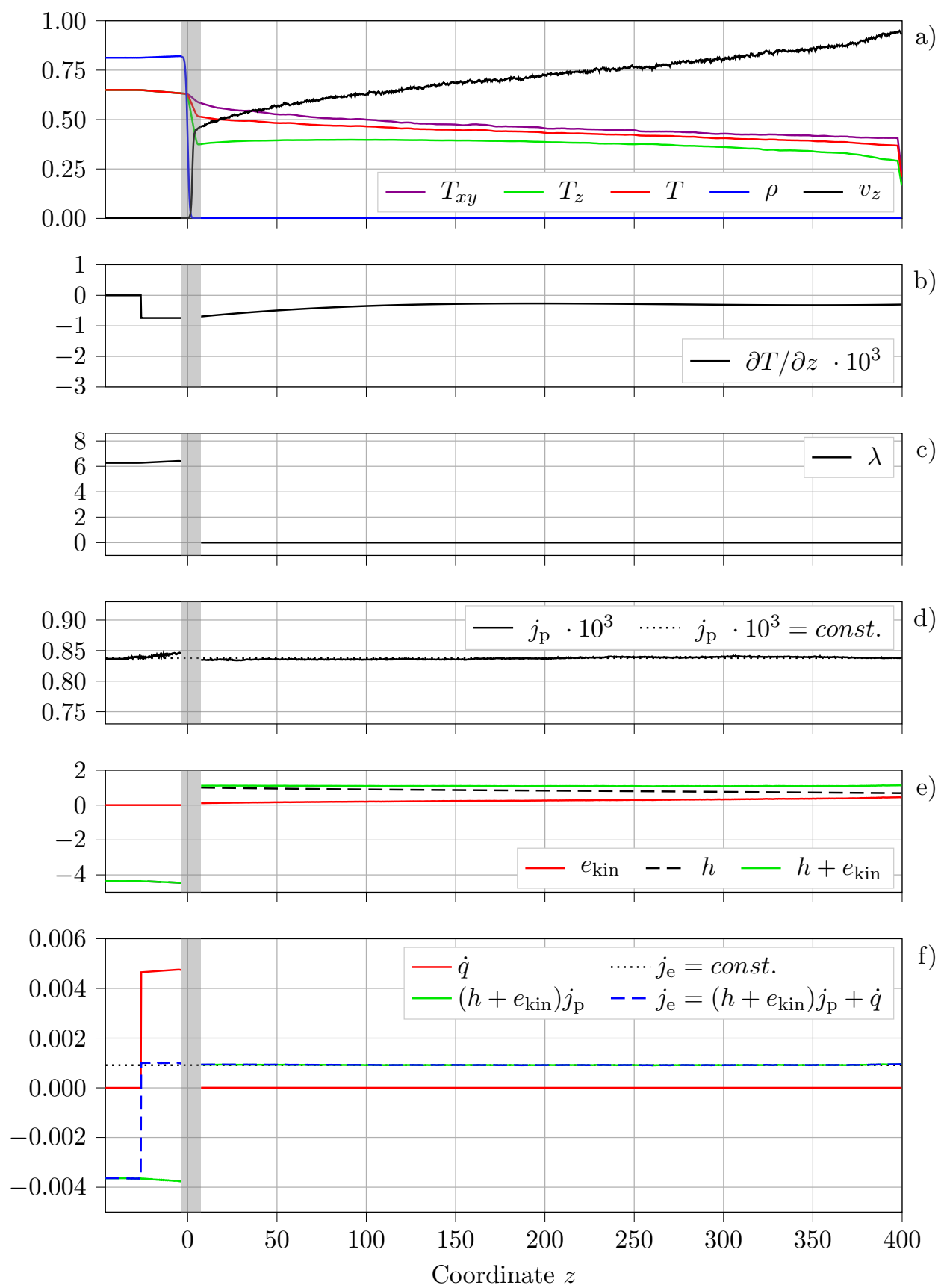

Figure 25. Evaluation of the energy flux $j_{\mathrm{e}}$ obtained by post-processing of profiles sampled during simulation with $T_{\text {liq }}=0.65$ and $L_{\mathrm{n}}=26$. Panels according to the description outlined above. 

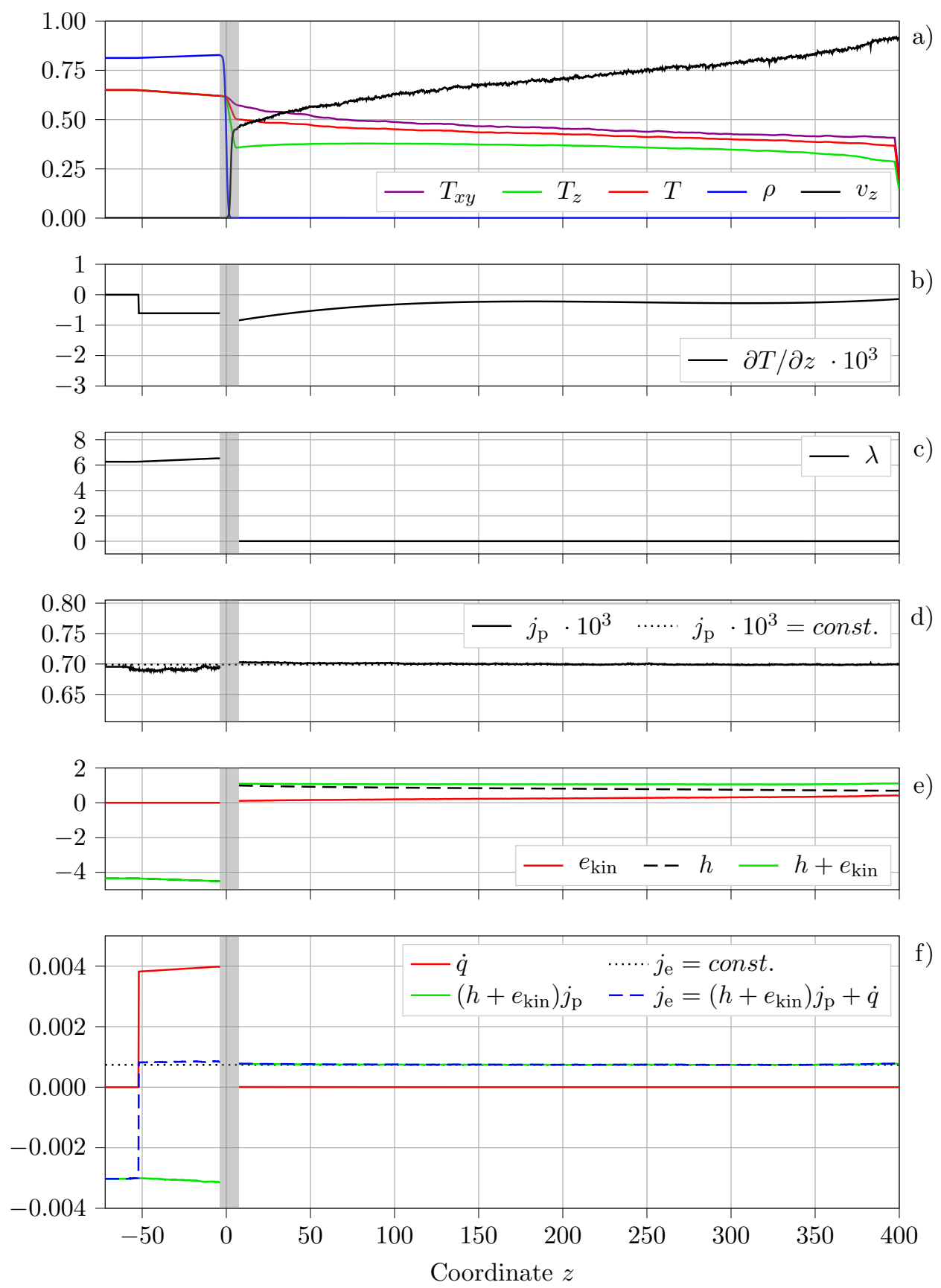

Figure 26. Evaluation of the energy flux $j_{\mathrm{e}}$ obtained by post-processing of profiles sampled during simulation with $T_{\text {liq }}=0.65$ and $L_{\mathrm{n}}=52$. Panels according to the description outlined above. 

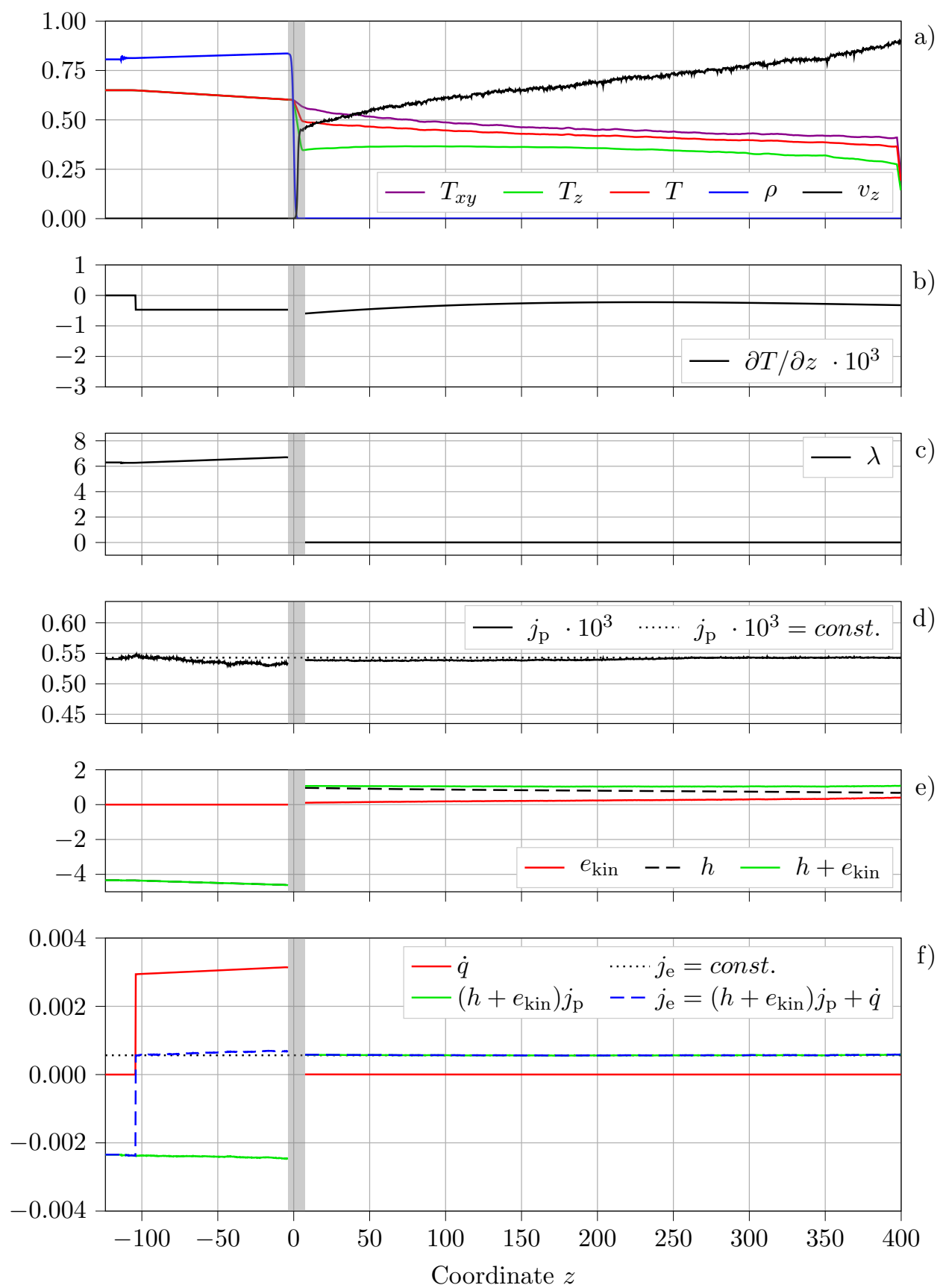

Figure 27. Evaluation of the energy flux $j_{\mathrm{e}}$ obtained by post-processing of profiles sampled during simulation with $T_{\text {liq }}=0.65$ and $L_{\mathrm{n}}=104$. Panels according to the description outlined above. 

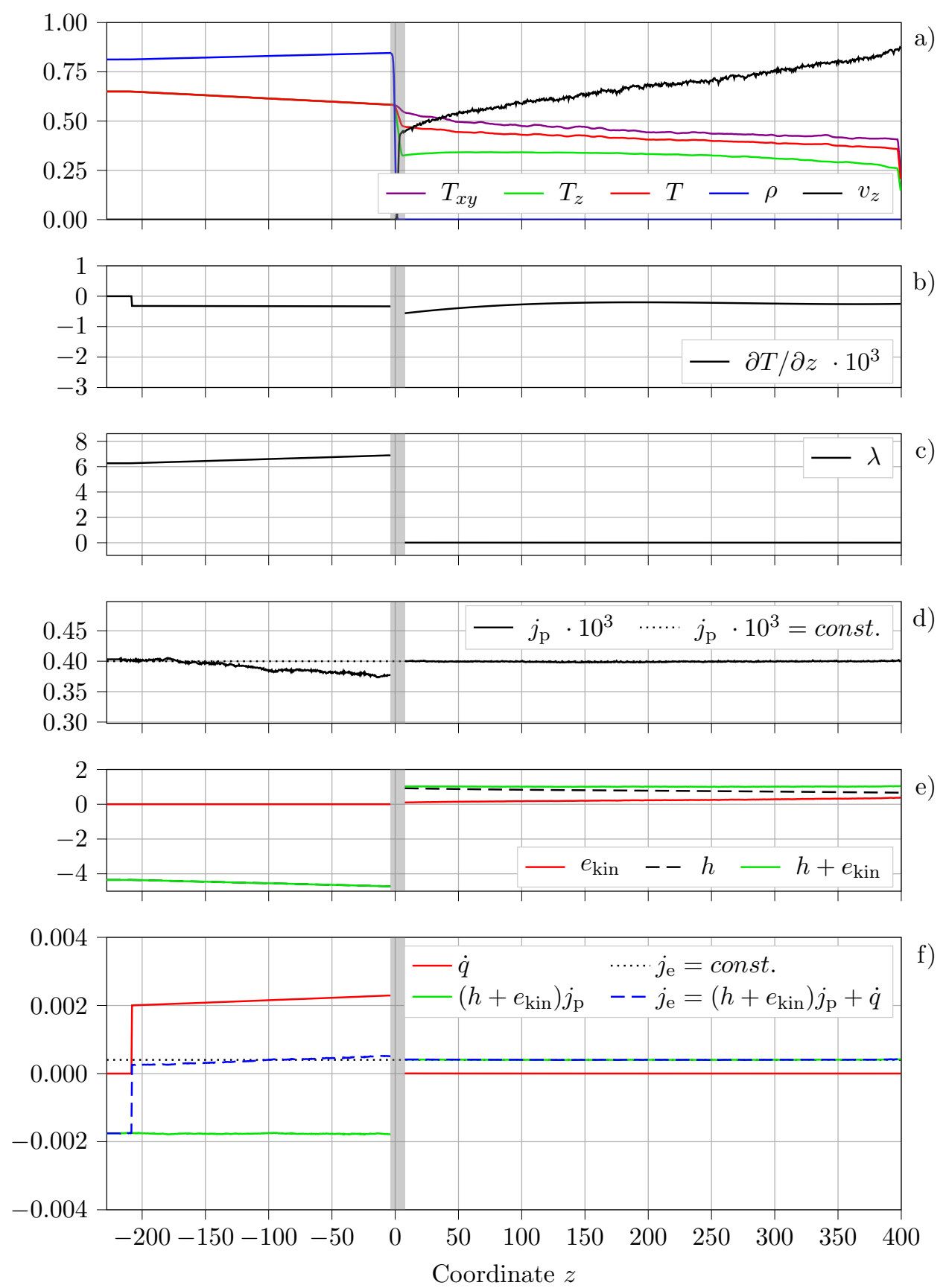

Figure 28. Evaluation of the energy flux $j_{\mathrm{e}}$ obtained by post-processing of profiles sampled during simulation with $T_{\text {liq }}=0.65$ and $L_{\mathrm{n}}=208$. Panels according to the description outlined above. 

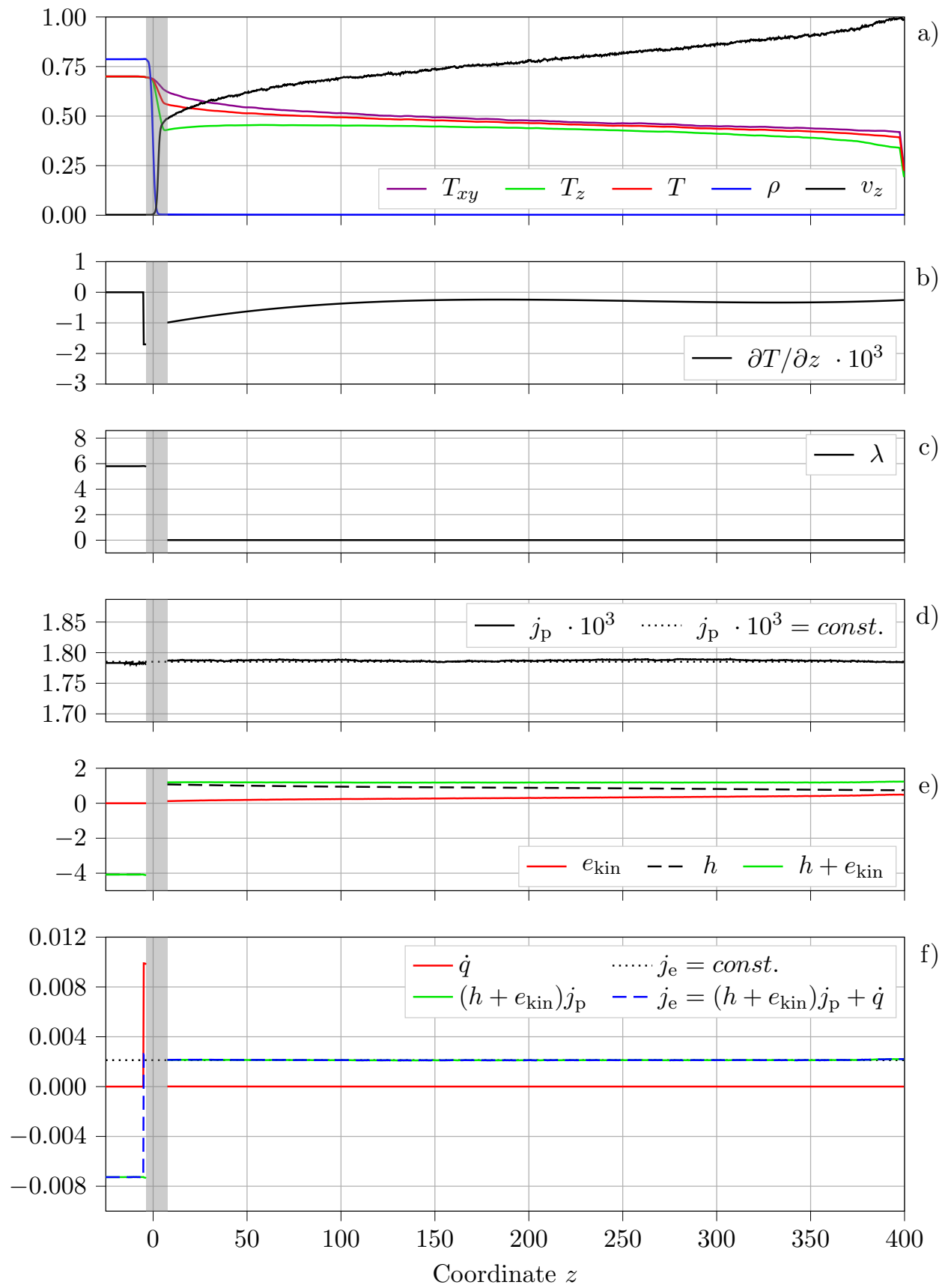

Figure 29. Evaluation of the energy flux $j_{\mathrm{e}}$ obtained by post-processing of profiles sampled during simulation with $T_{\text {liq }}=0.7$ and $L_{\mathrm{n}}=5.2$. Panels according to the description outlined above. 

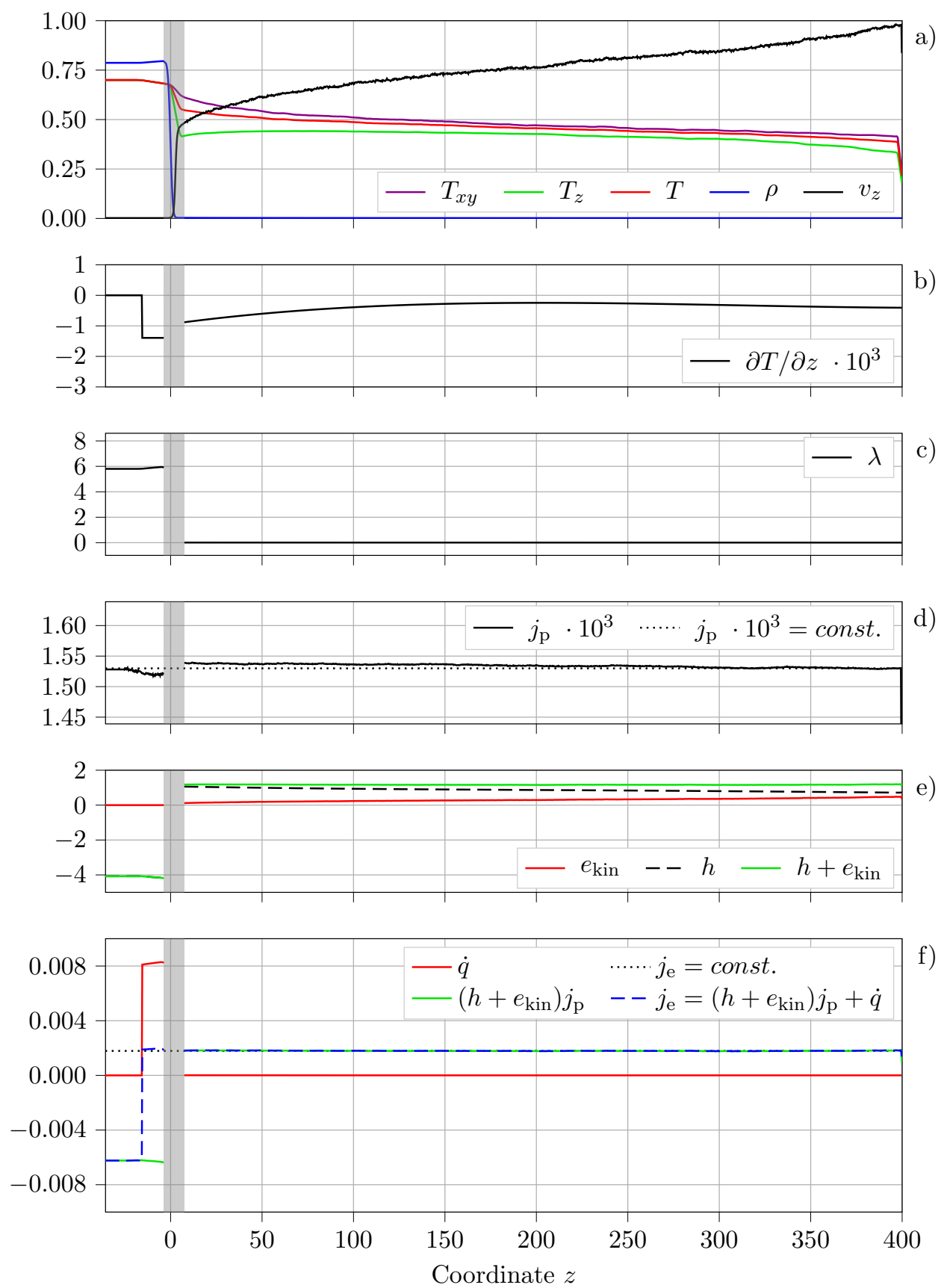

Figure 30. Evaluation of the energy flux $j_{\mathrm{e}}$ obtained by post-processing of profiles sampled during simulation with $T_{\text {liq }}=0.7$ and $L_{\mathrm{n}}=15.6$. Panels according to the description outlined above. 

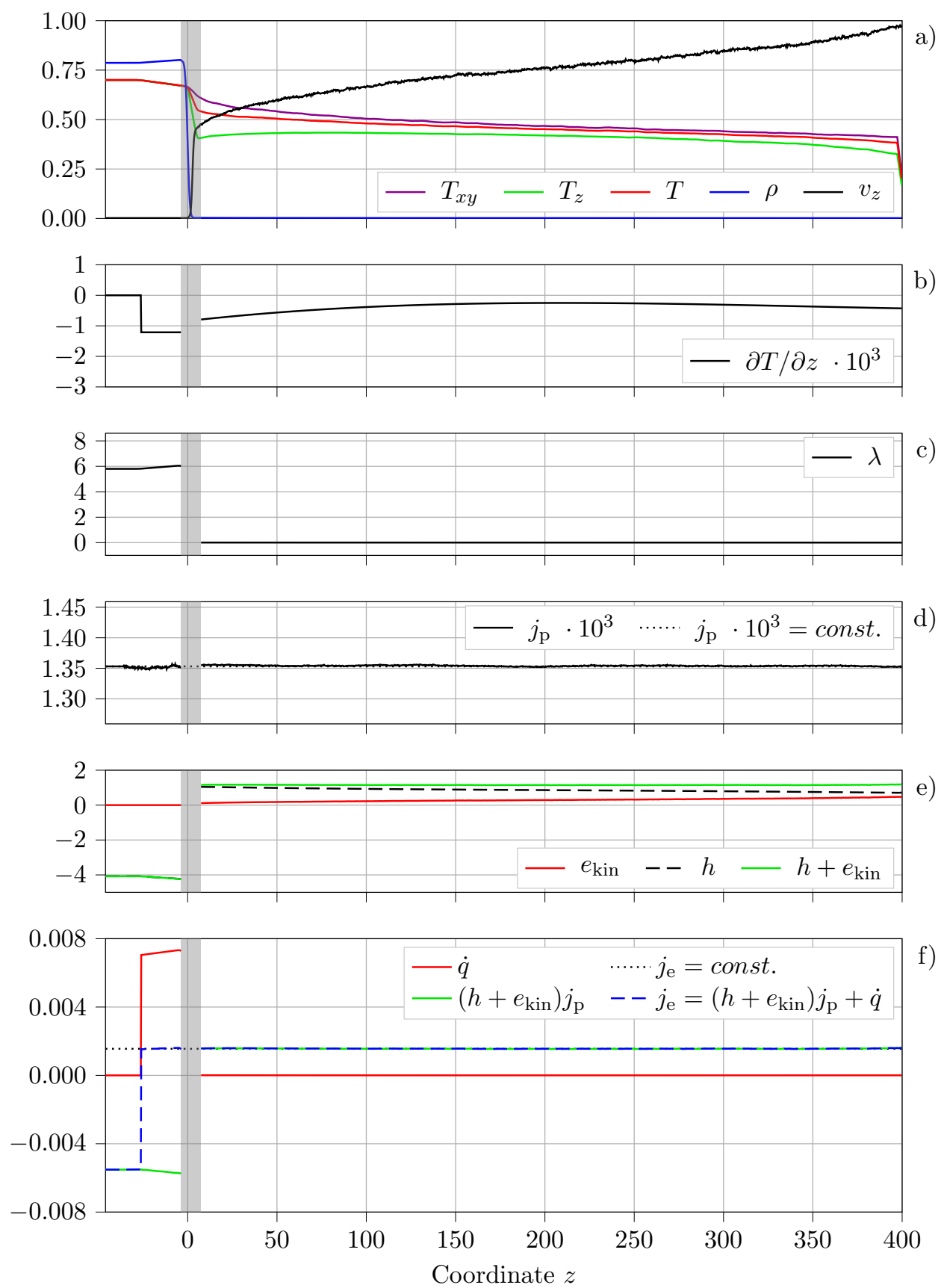

Figure 31. Evaluation of the energy flux $j_{\mathrm{e}}$ obtained by post-processing of profiles sampled during simulation with $T_{\text {liq }}=0.7$ and $L_{\mathrm{n}}=26$. Panels according to the description outlined above. 

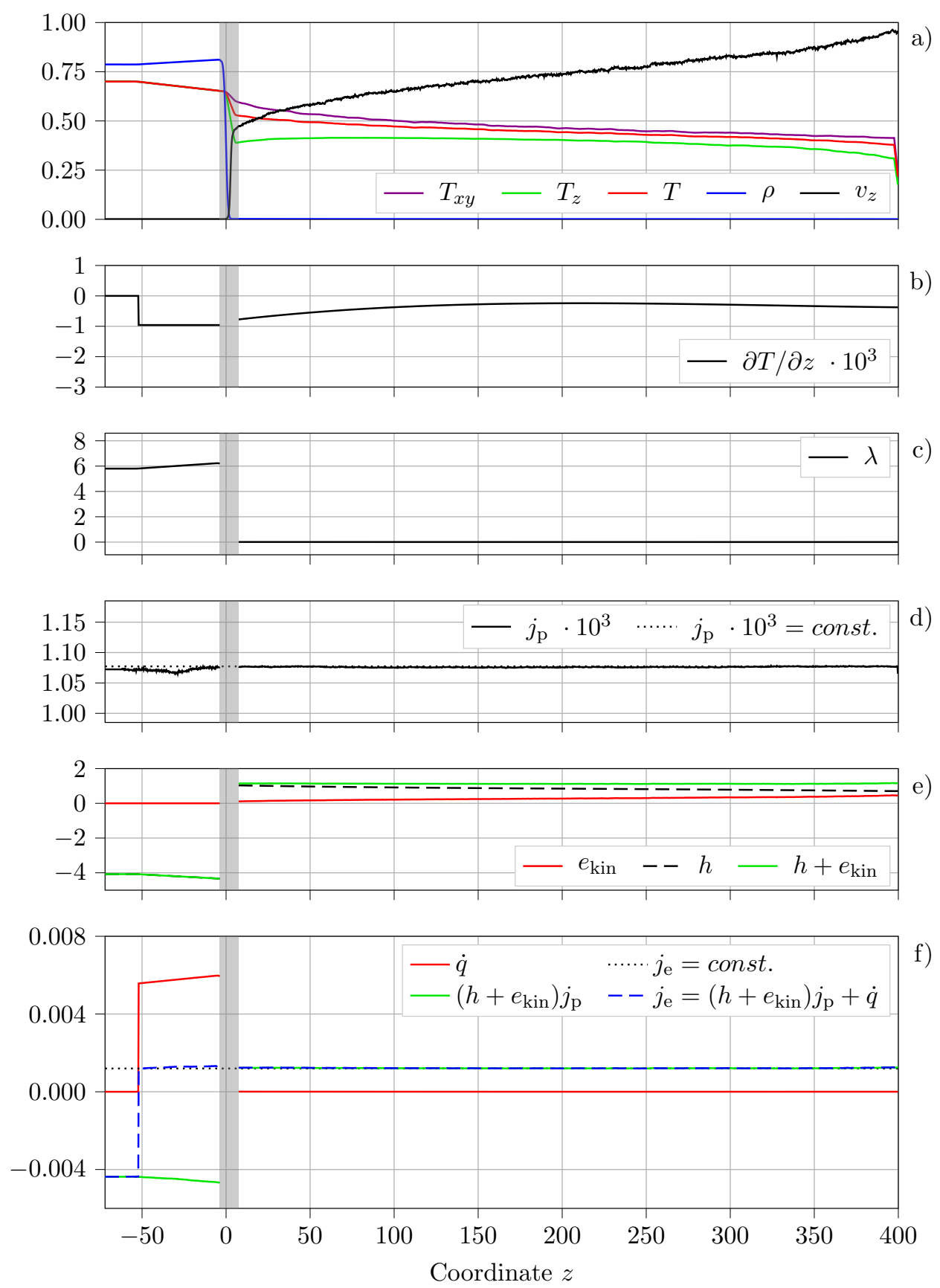

Figure 32. Evaluation of the energy flux $j_{\mathrm{e}}$ obtained by post-processing of profiles sampled during simulation with $T_{\text {liq }}=0.7$ and $L_{\mathrm{n}}=52$. Panels according to the description outlined above. 

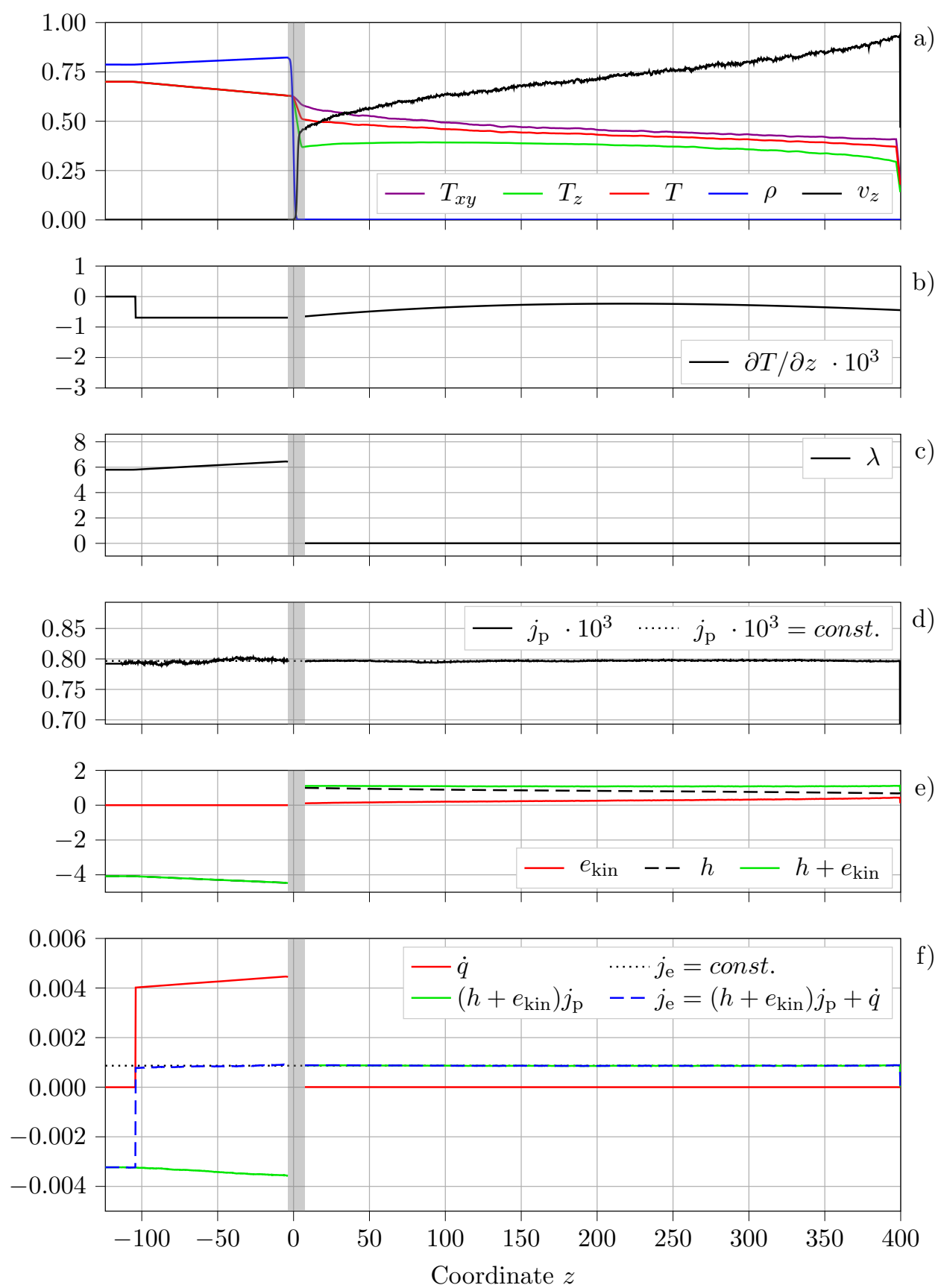

Figure 33. Evaluation of the energy flux $j_{\mathrm{e}}$ obtained by post-processing of profiles sampled during simulation with $T_{\text {liq }}=0.7$ and $L_{\mathrm{n}}=104$. Panels according to the description outlined above. 

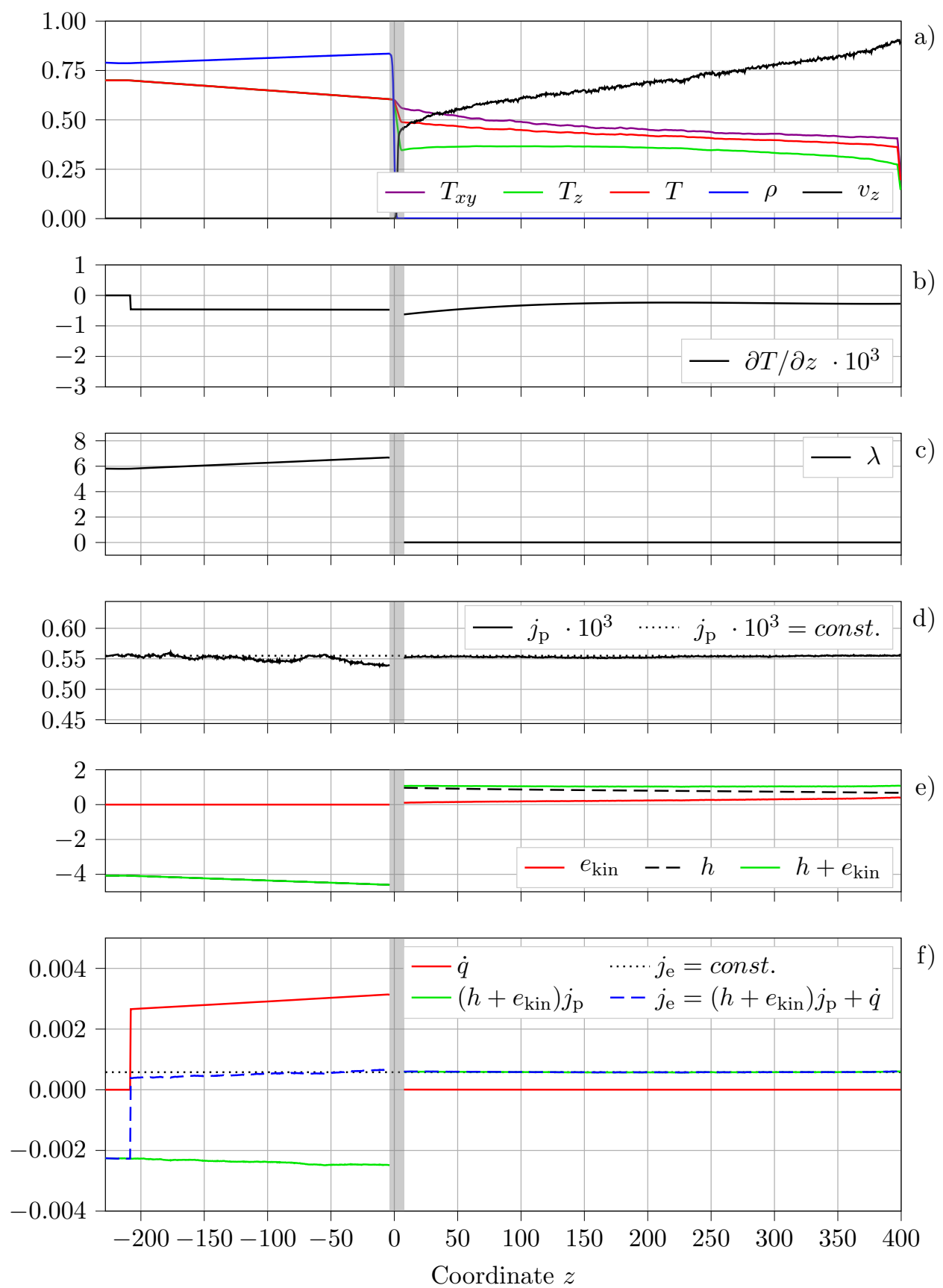

Figure 34. Evaluation of the energy flux $j_{\mathrm{e}}$ obtained by post-processing of profiles sampled during simulation with $T_{\text {liq }}=0.7$ and $L_{\mathrm{n}}=208$. Panels according to the description outlined above. 


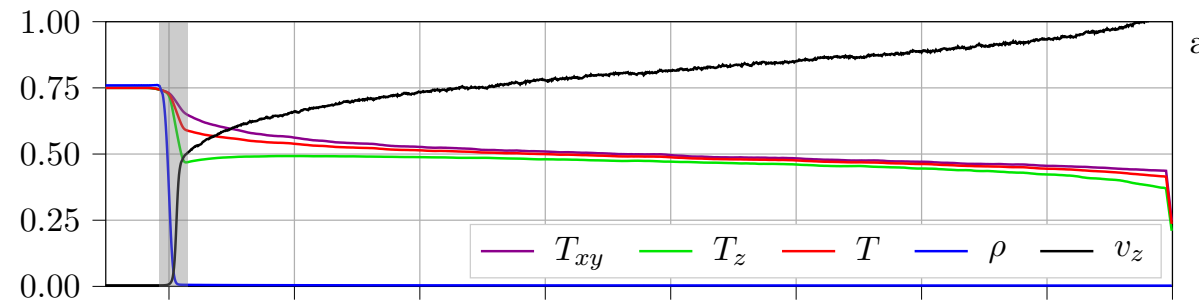

a)
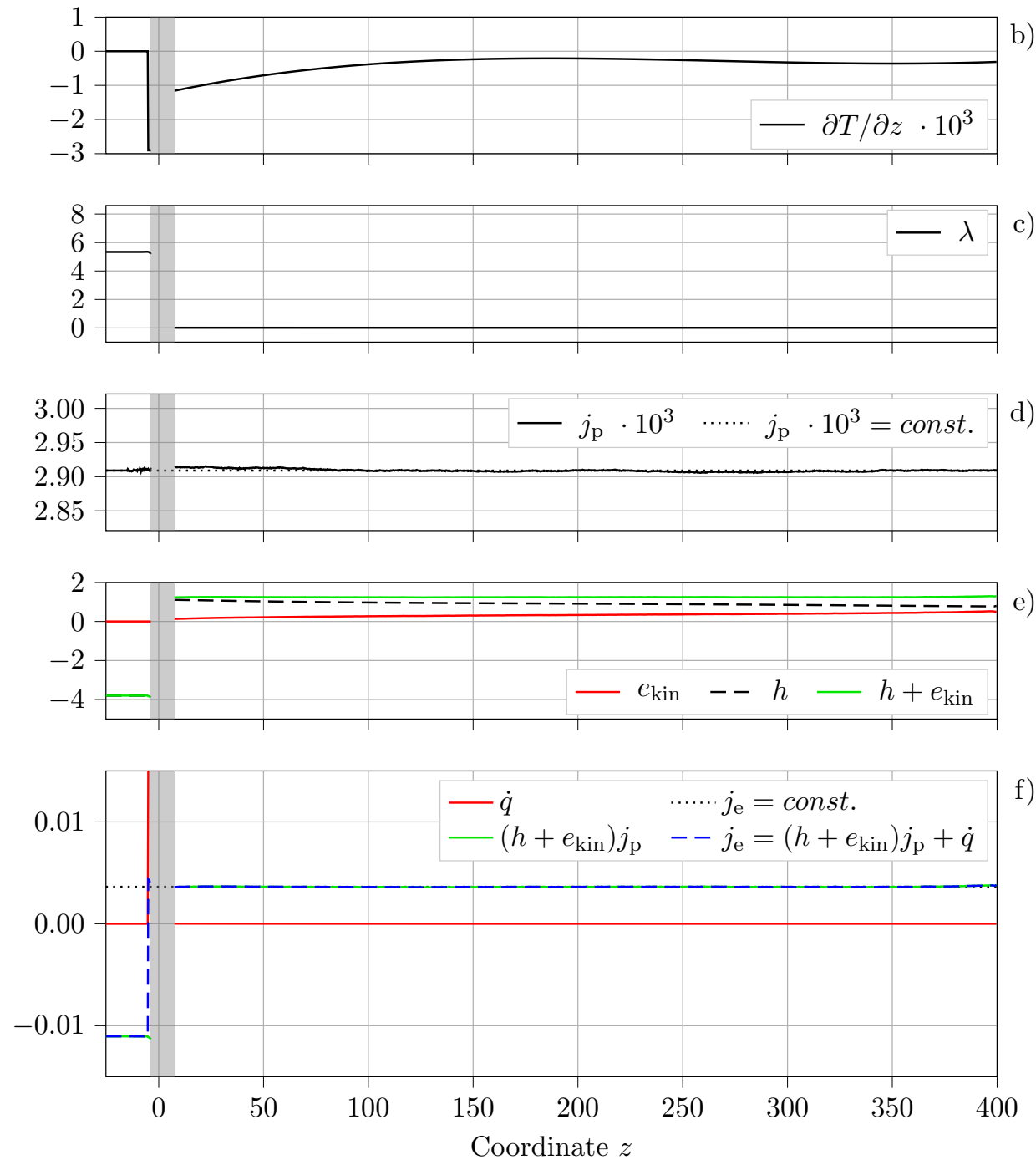

Figure 35. Evaluation of the energy flux $j_{\mathrm{e}}$ obtained by post-processing of profiles sampled during simulation with $T_{\text {liq }}=0.75$ and $L_{\mathrm{n}}=5.2$. Panels according to the description outlined above. 


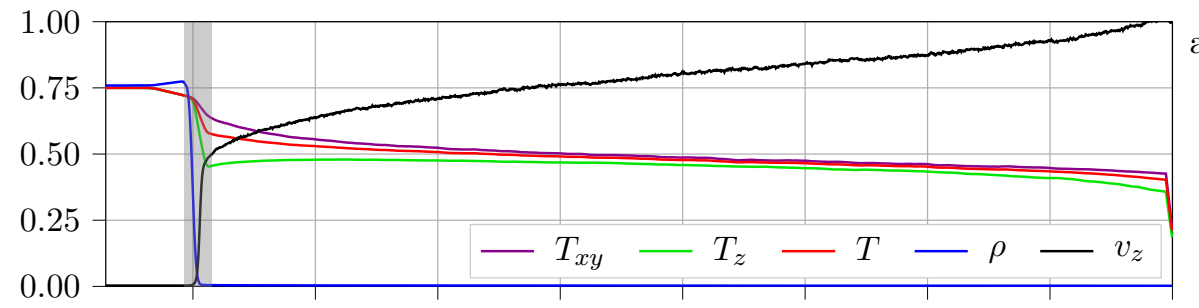

a)

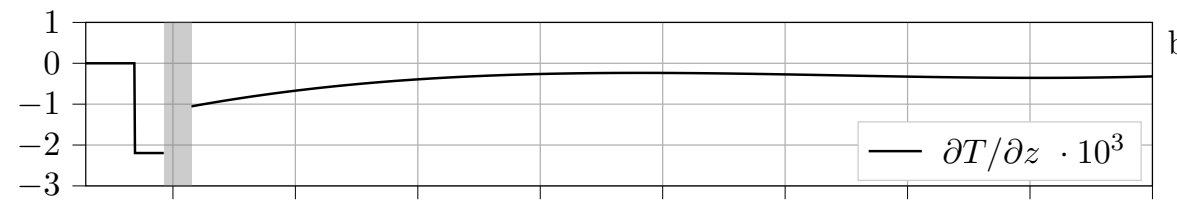

\section{b)}
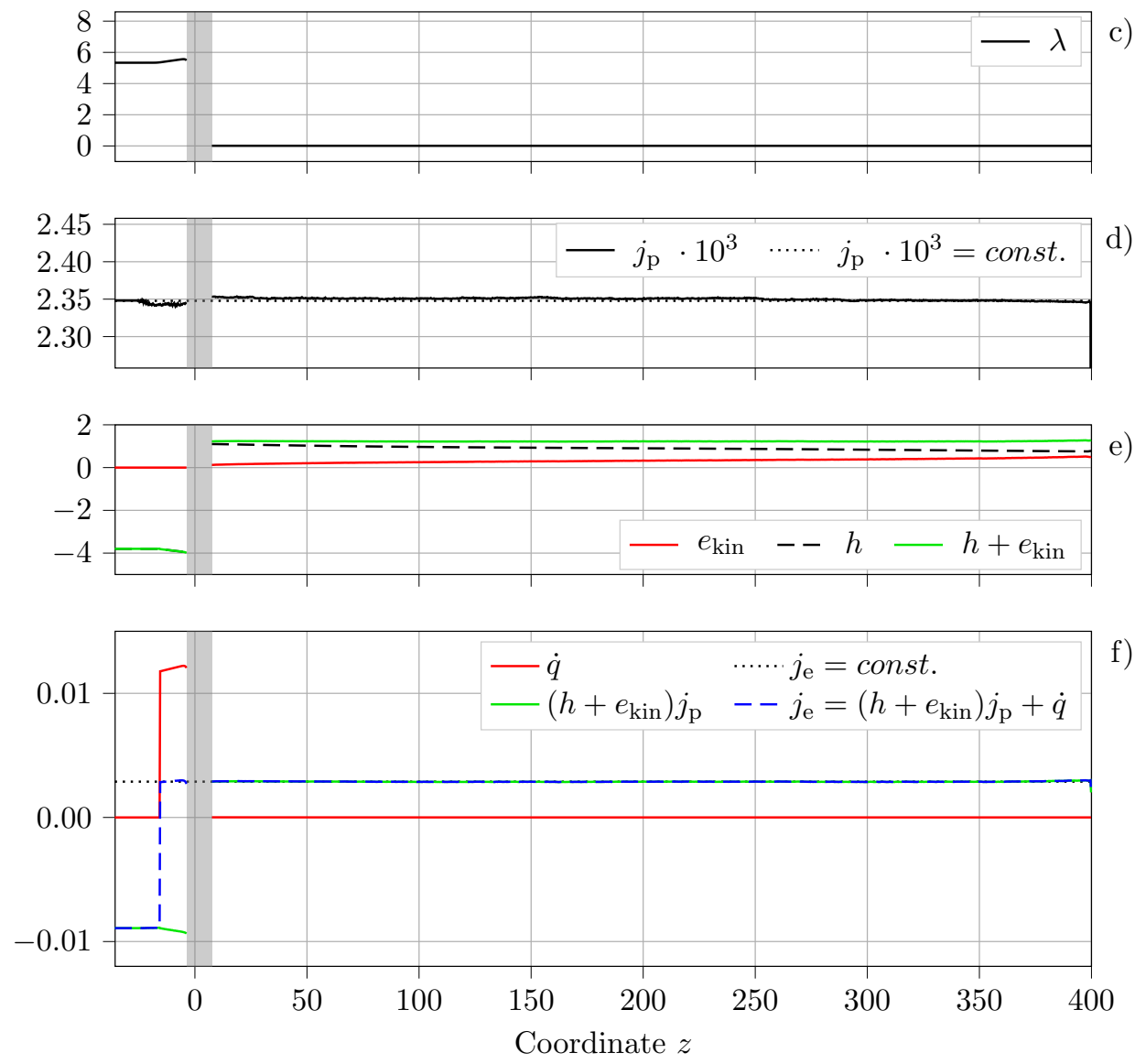

Figure 36. Evaluation of the energy flux $j_{\mathrm{e}}$ obtained by post-processing of profiles sampled during simulation with $T_{\text {liq }}=0.75$ and $L_{\mathrm{n}}=15.6$. Panels according to the description outlined above. 


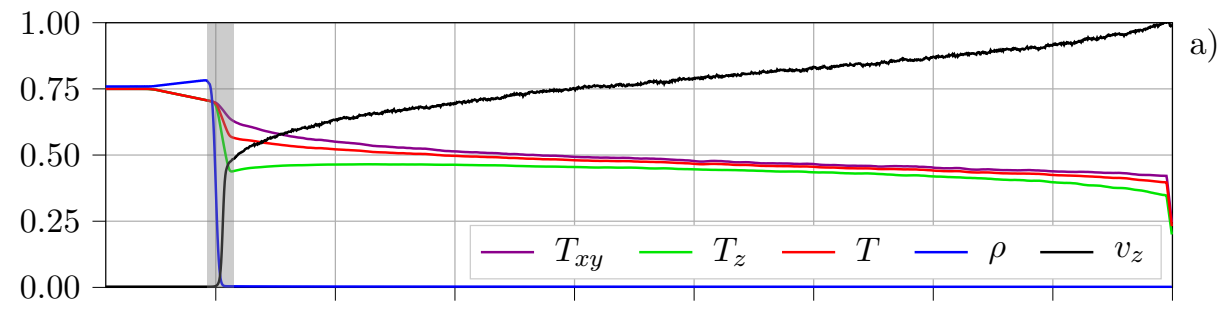

a)

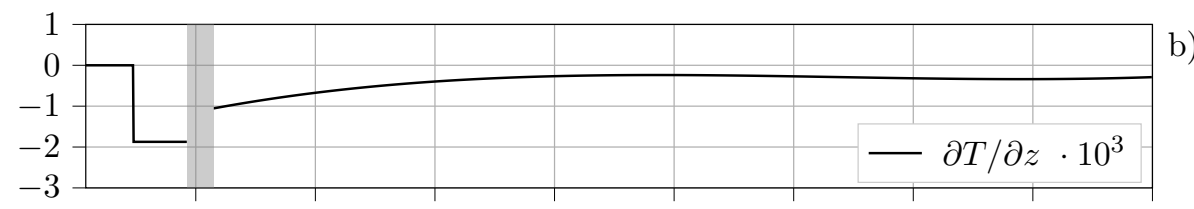

$$
\text { b) }
$$
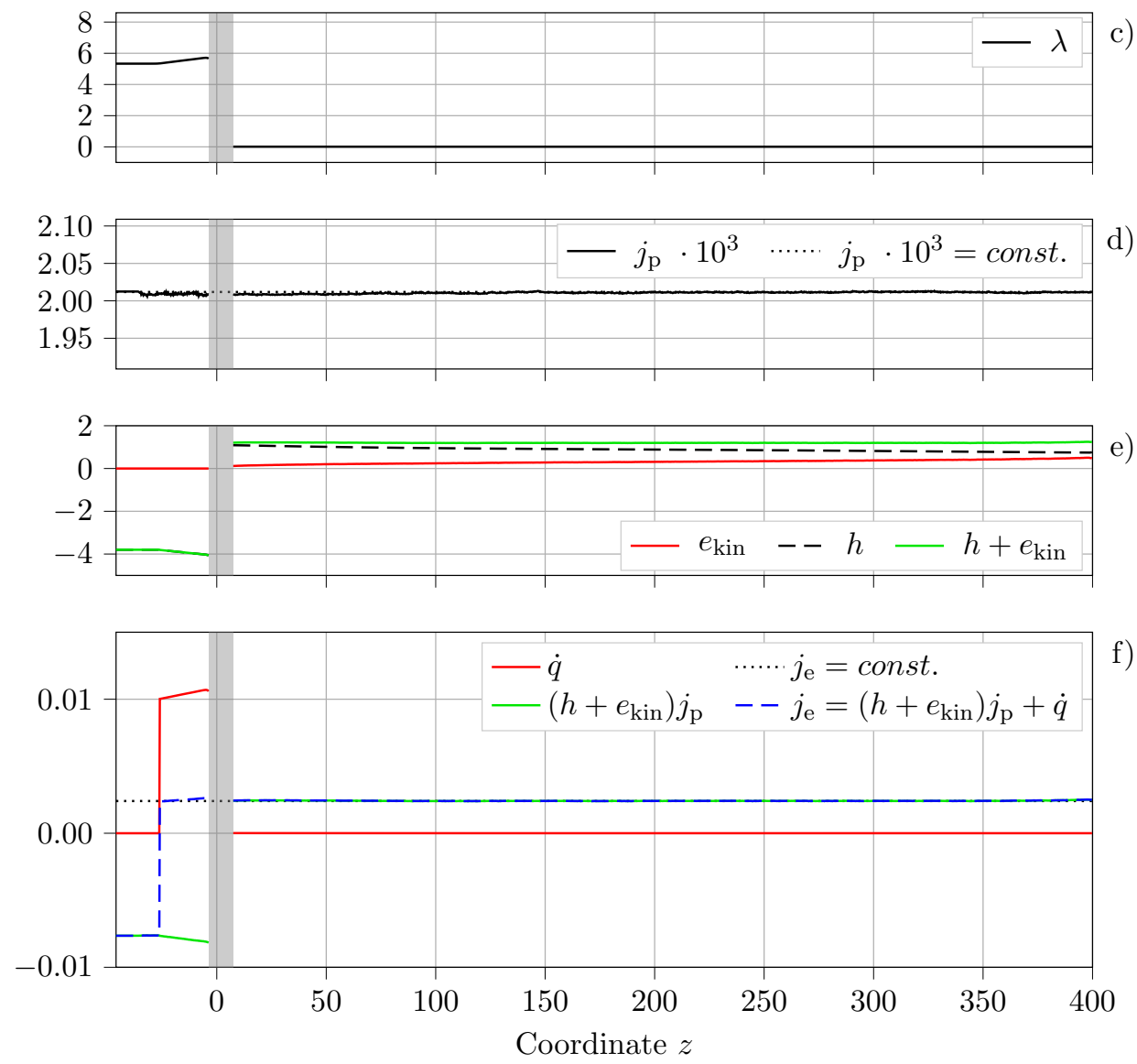

Figure 37. Evaluation of the energy flux $j_{\mathrm{e}}$ obtained by post-processing of profiles sampled during simulation with $T_{\text {liq }}=0.75$ and $L_{\mathrm{n}}=26$. Panels according to the description outlined above. 

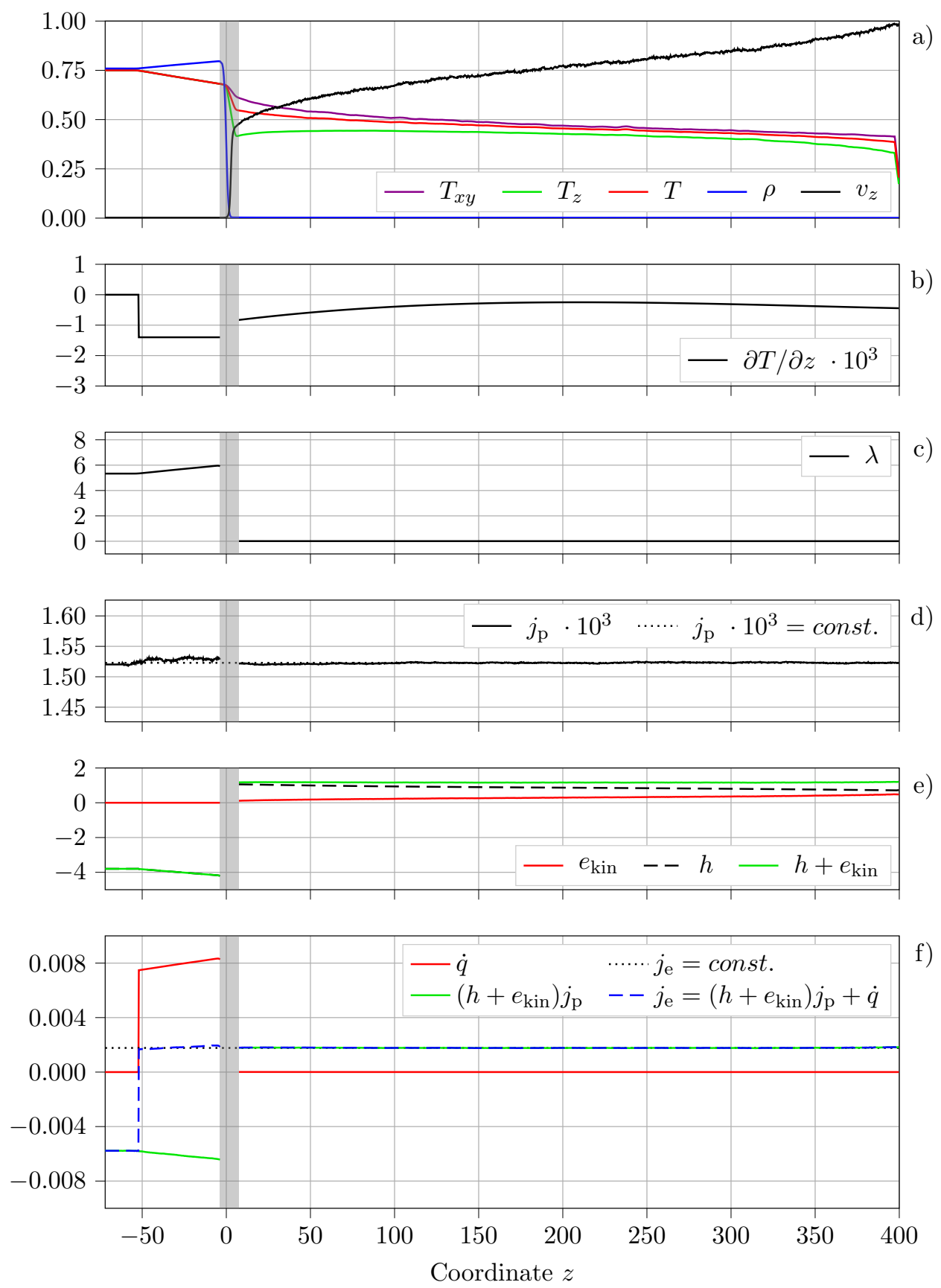

Figure 38. Evaluation of the energy flux $j_{\mathrm{e}}$ obtained by post-processing of profiles sampled during simulation with $T_{\text {liq }}=0.75$ and $L_{\mathrm{n}}=52$. Panels according to the description outlined above. 

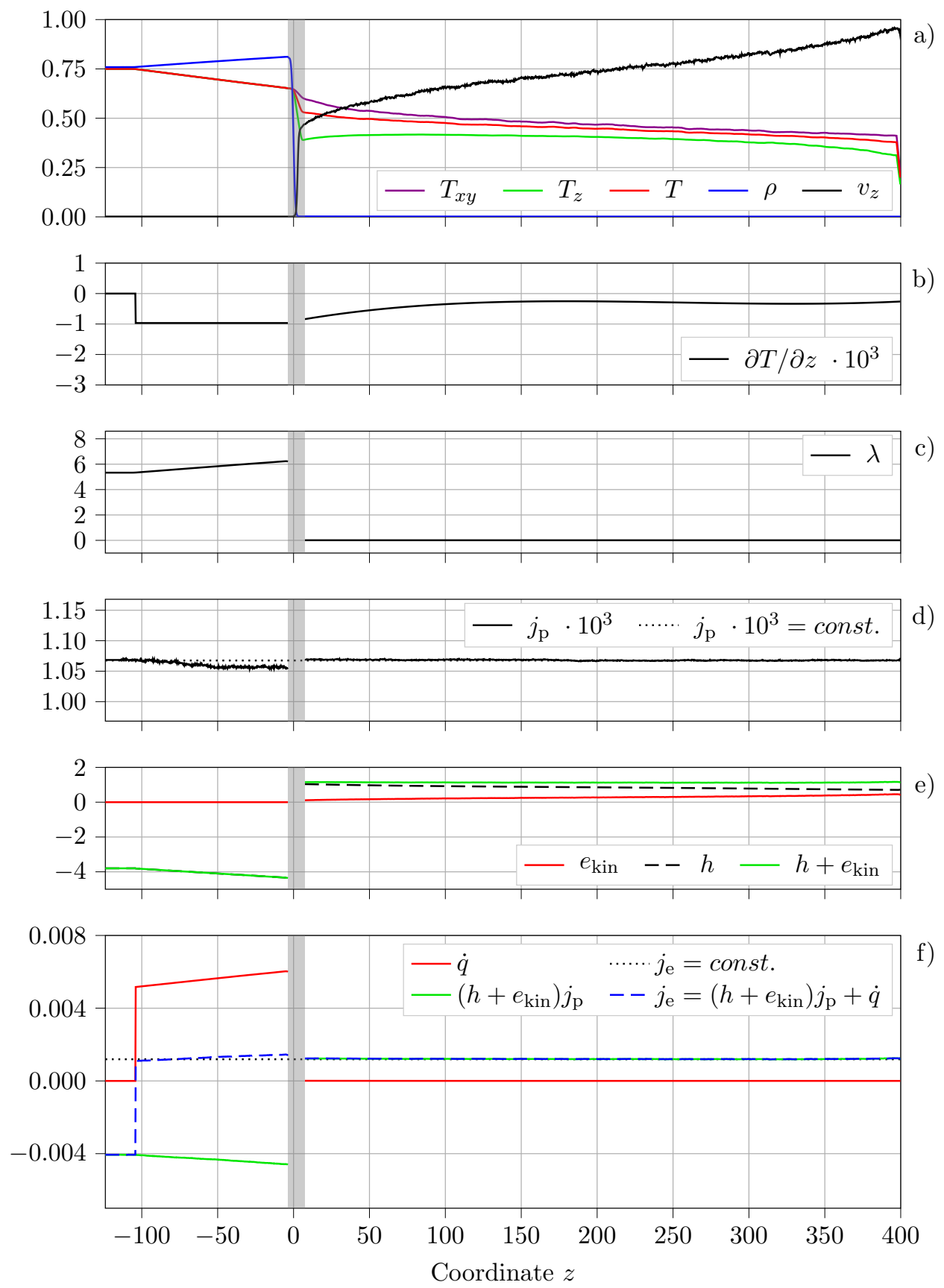

Figure 39. Evaluation of the energy flux $j_{\mathrm{e}}$ obtained by post-processing of profiles sampled during simulation with $T_{\text {liq }}=0.75$ and $L_{\mathrm{n}}=104$. Panels according to the description outlined above. 

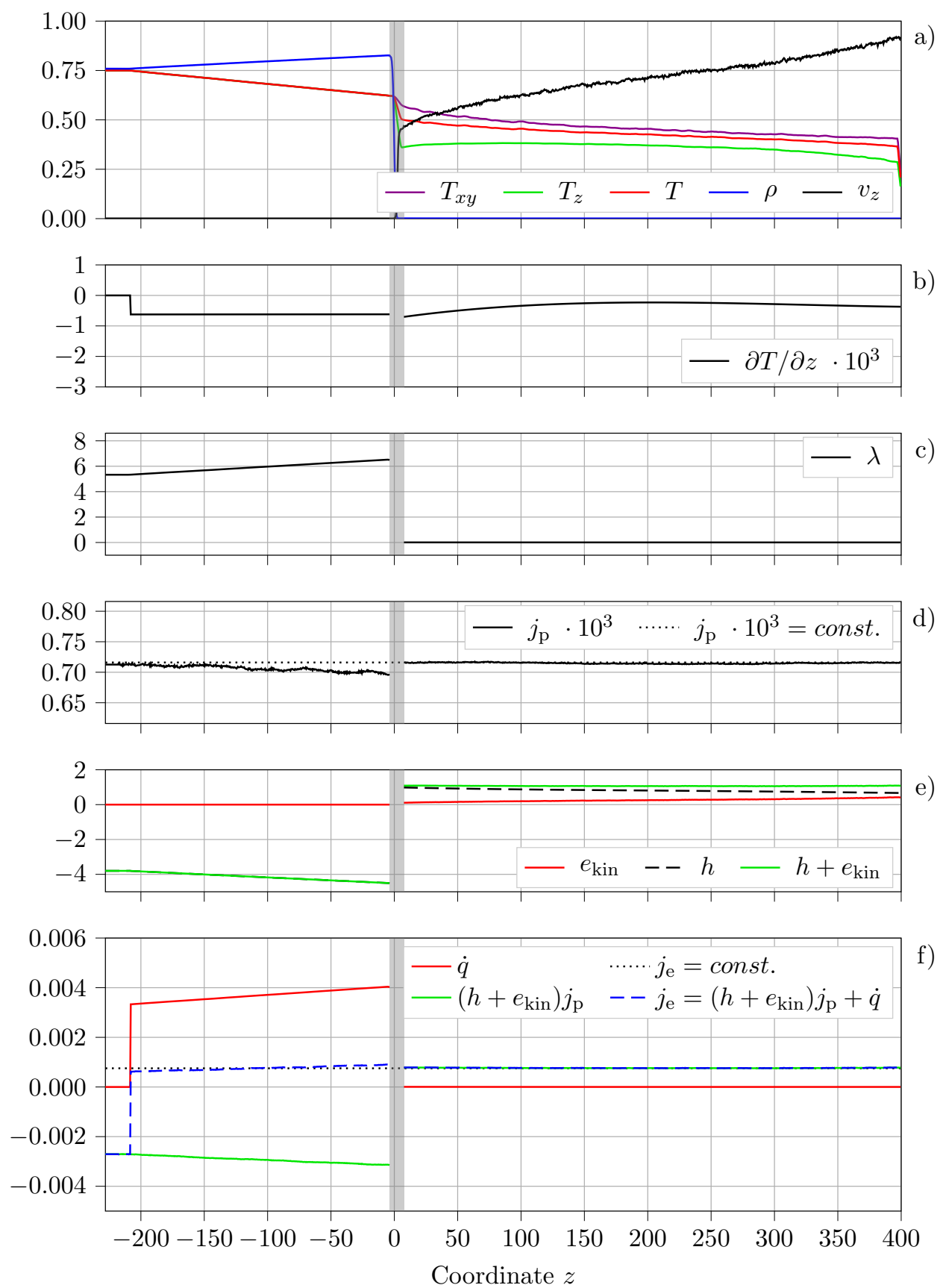

Figure 40. Evaluation of the energy flux $j_{\mathrm{e}}$ obtained by post-processing of profiles sampled during simulation with $T_{\text {liq }}=0.75$ and $L_{\mathrm{n}}=208$. Panels according to the description outlined above. 


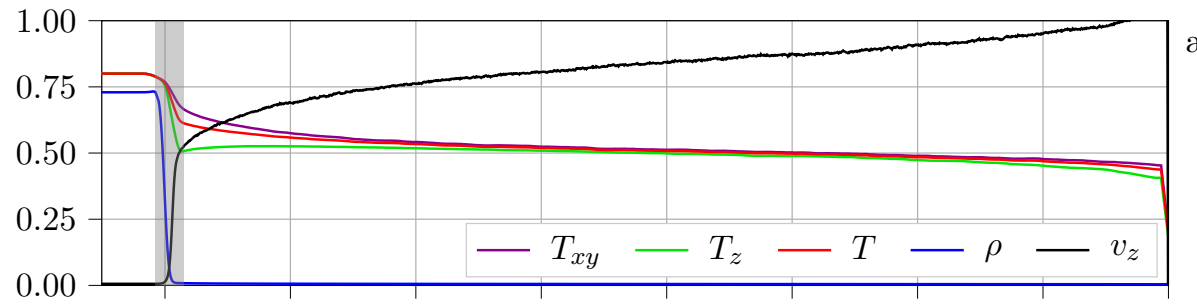

a)
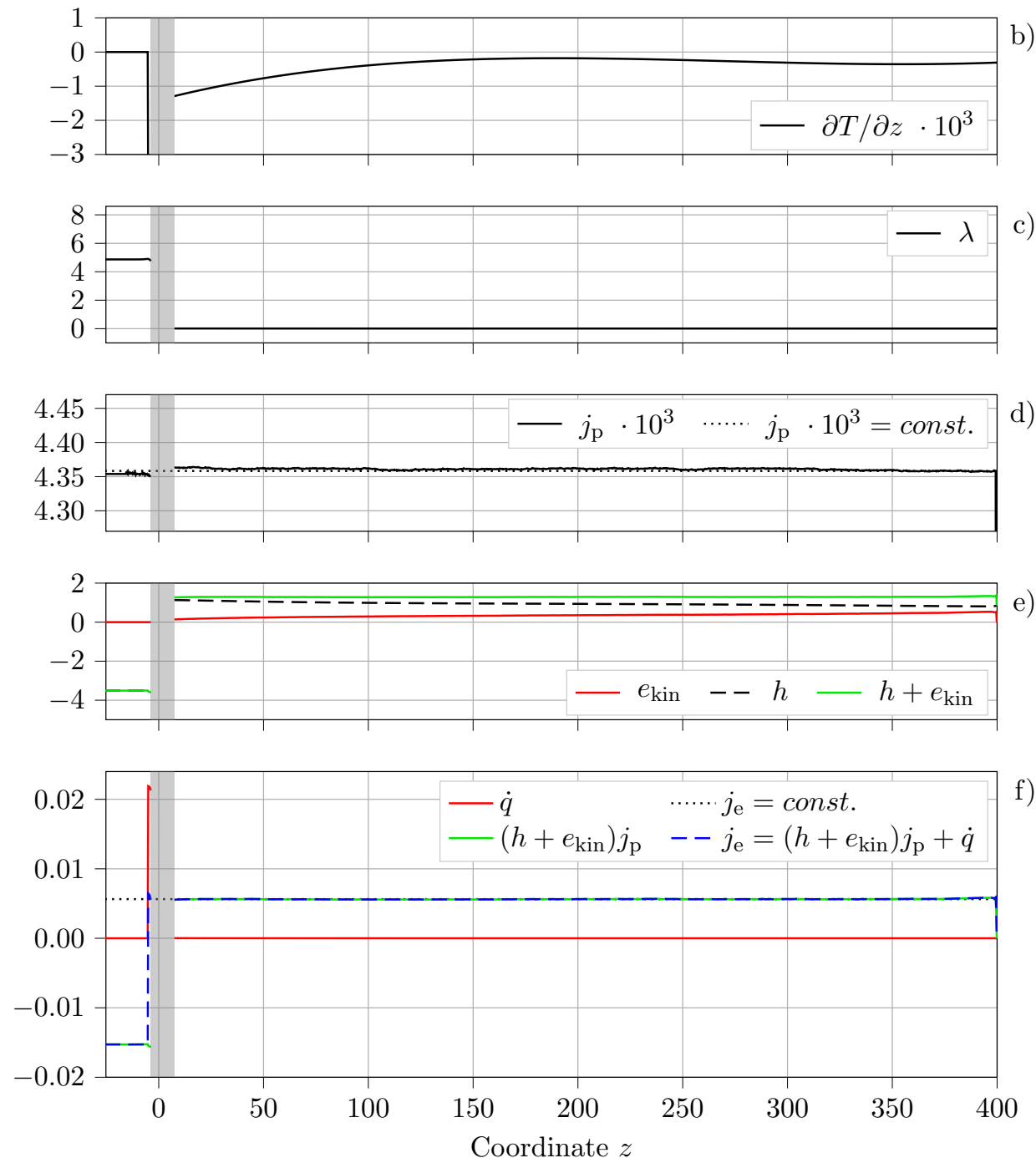

Figure 41. Evaluation of the energy flux $j_{\mathrm{e}}$ obtained by post-processing of profiles sampled during simulation with $T_{\text {liq }}=0.8$ and $L_{\mathrm{n}}=5.2$. Panels according to the description outlined above. 


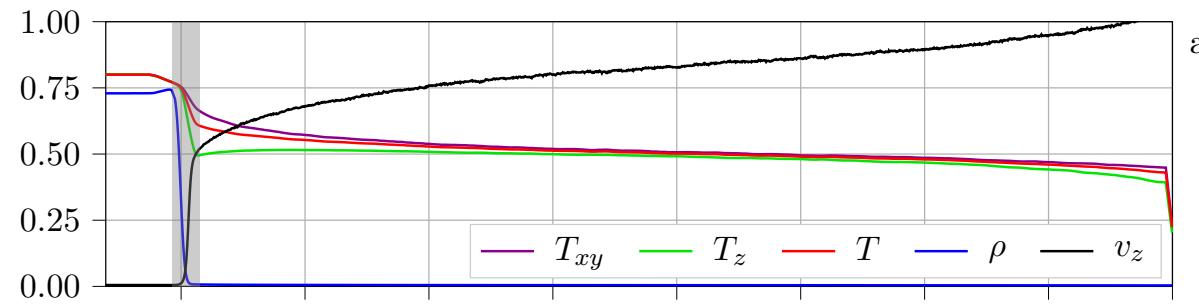

a)
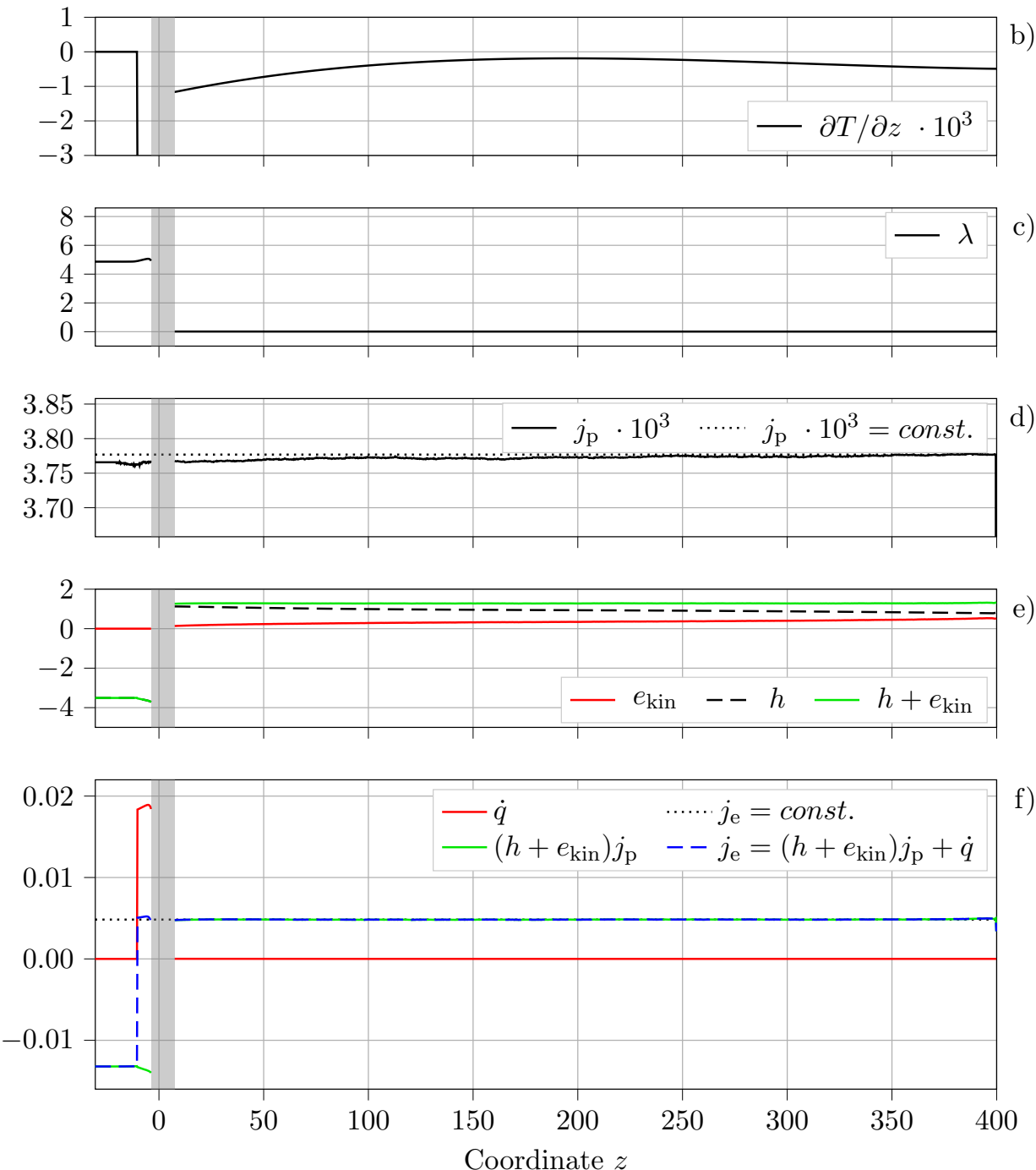

Figure 42. Evaluation of the energy flux $j_{\mathrm{e}}$ obtained by post-processing of profiles sampled during simulation with $T_{\text {liq }}=0.8$ and $L_{\mathrm{n}}=10.4$. Panels according to the description outlined above. 


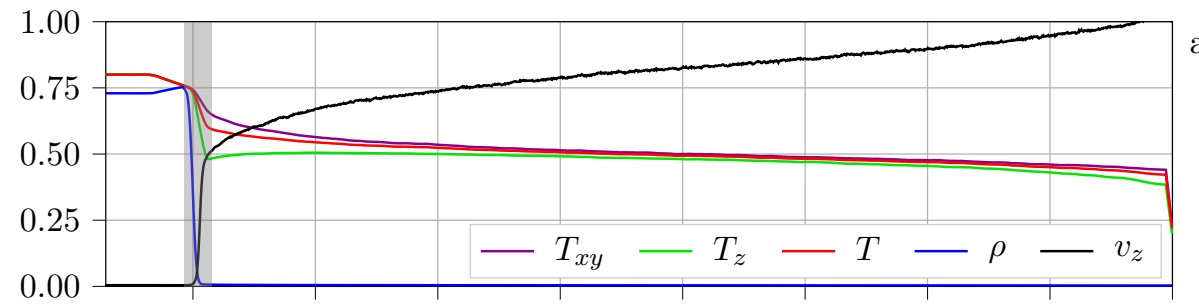

a)
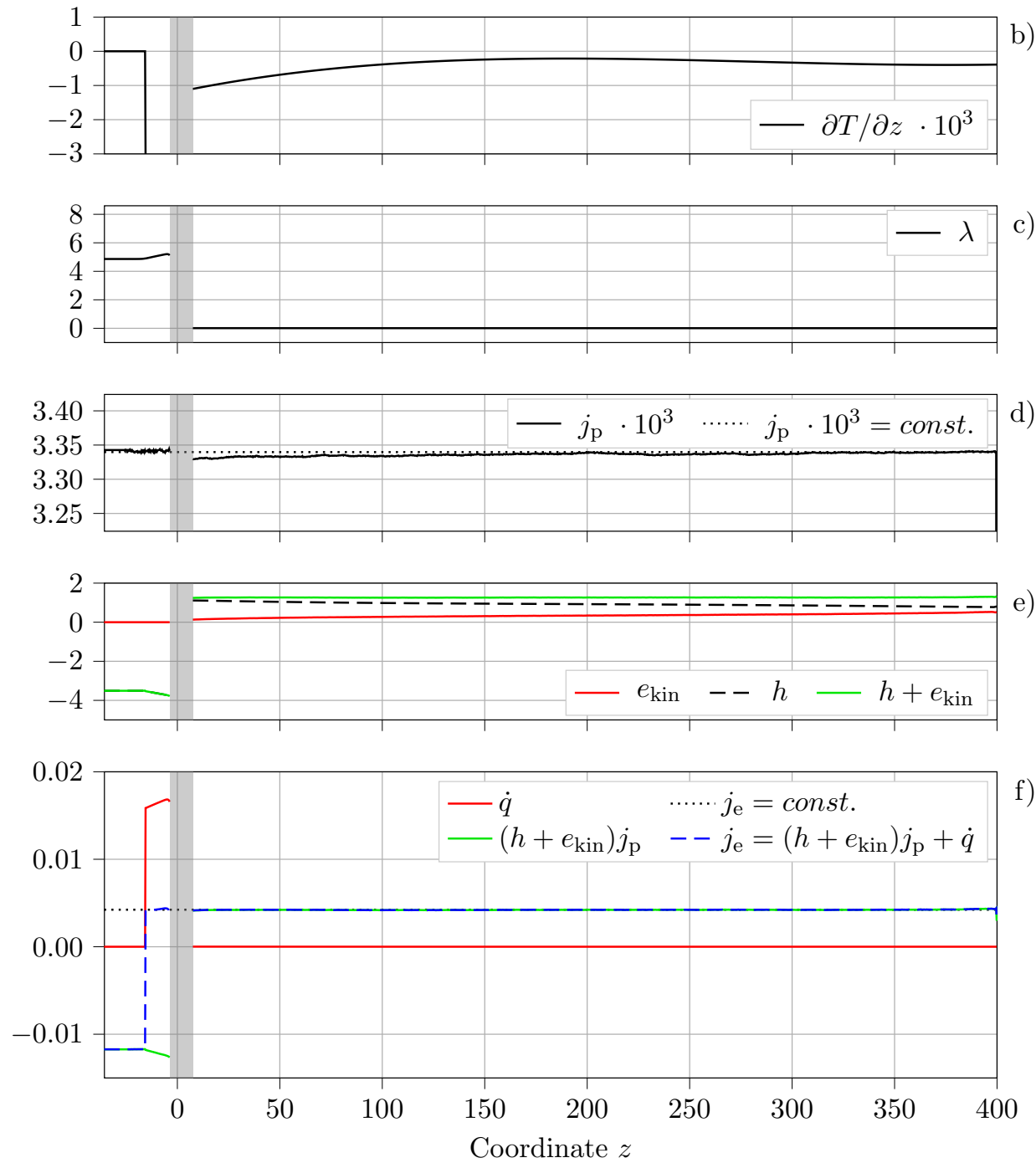

Figure 43. Evaluation of the energy flux $j_{\mathrm{e}}$ obtained by post-processing of profiles sampled during simulation with $T_{\text {liq }}=0.8$ and $L_{\mathrm{n}}=15.6$. Panels according to the description outlined above. 

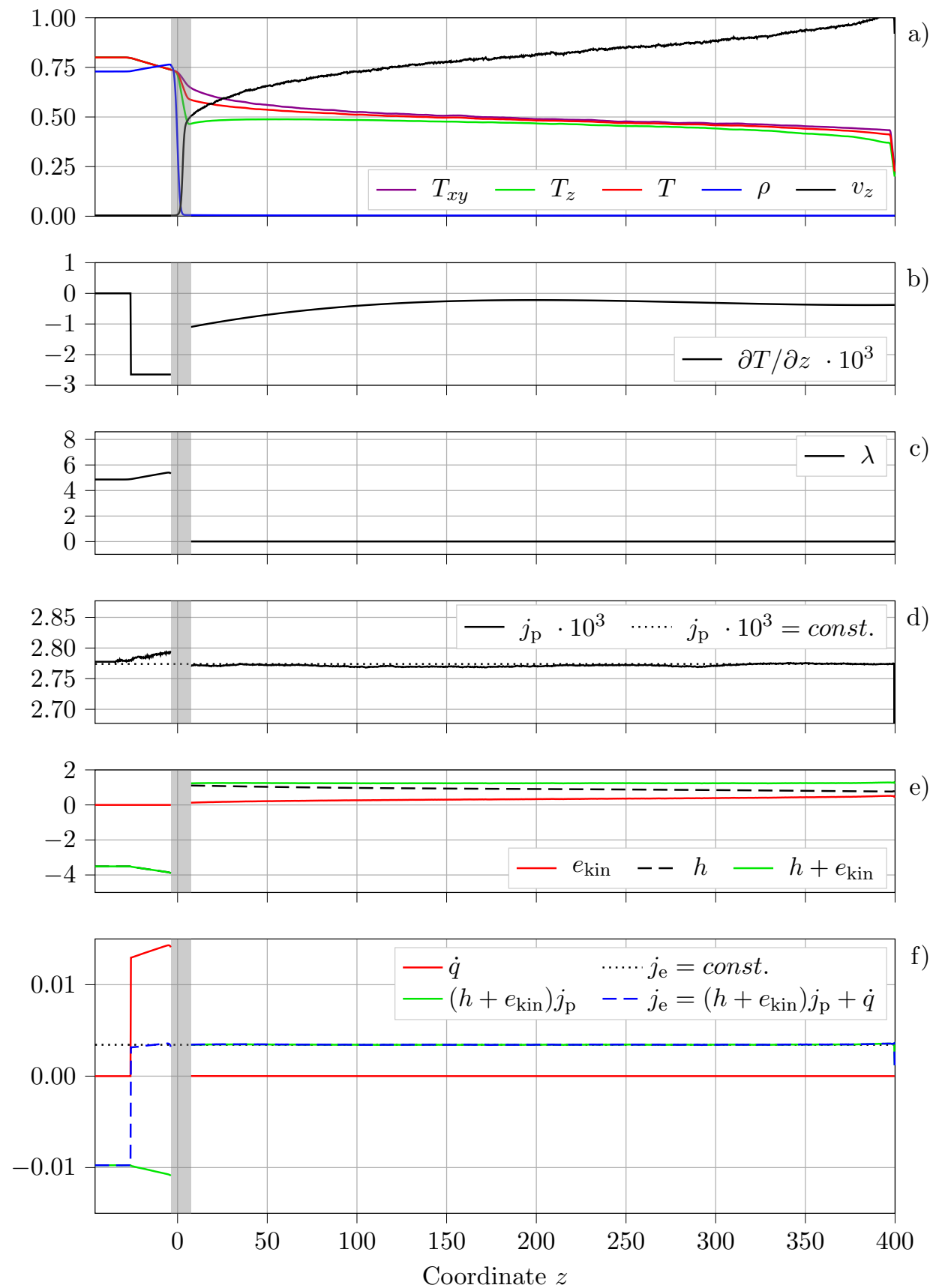

Figure 44. Evaluation of the energy flux $j_{\mathrm{e}}$ obtained by post-processing of profiles sampled during simulation with $T_{\text {liq }}=0.8$ and $L_{\mathrm{n}}=26$. Panels according to the description outlined above. 


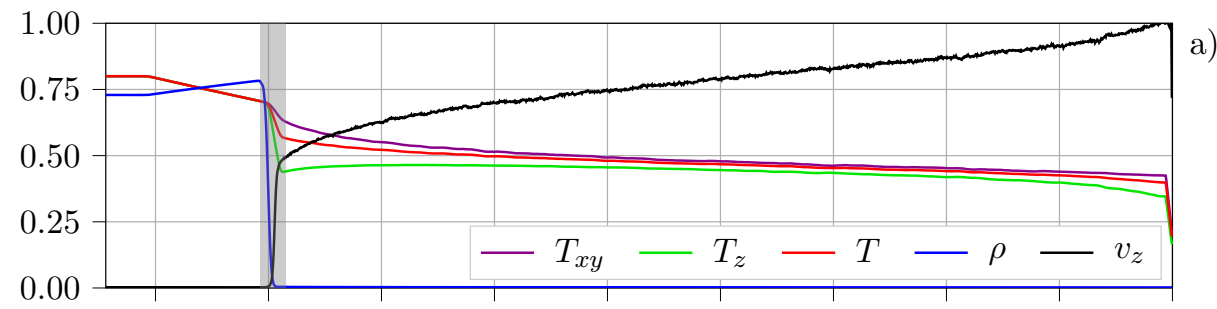

a)

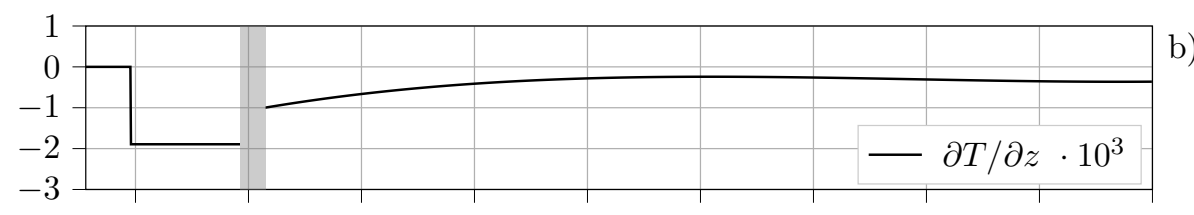

\section{b)}
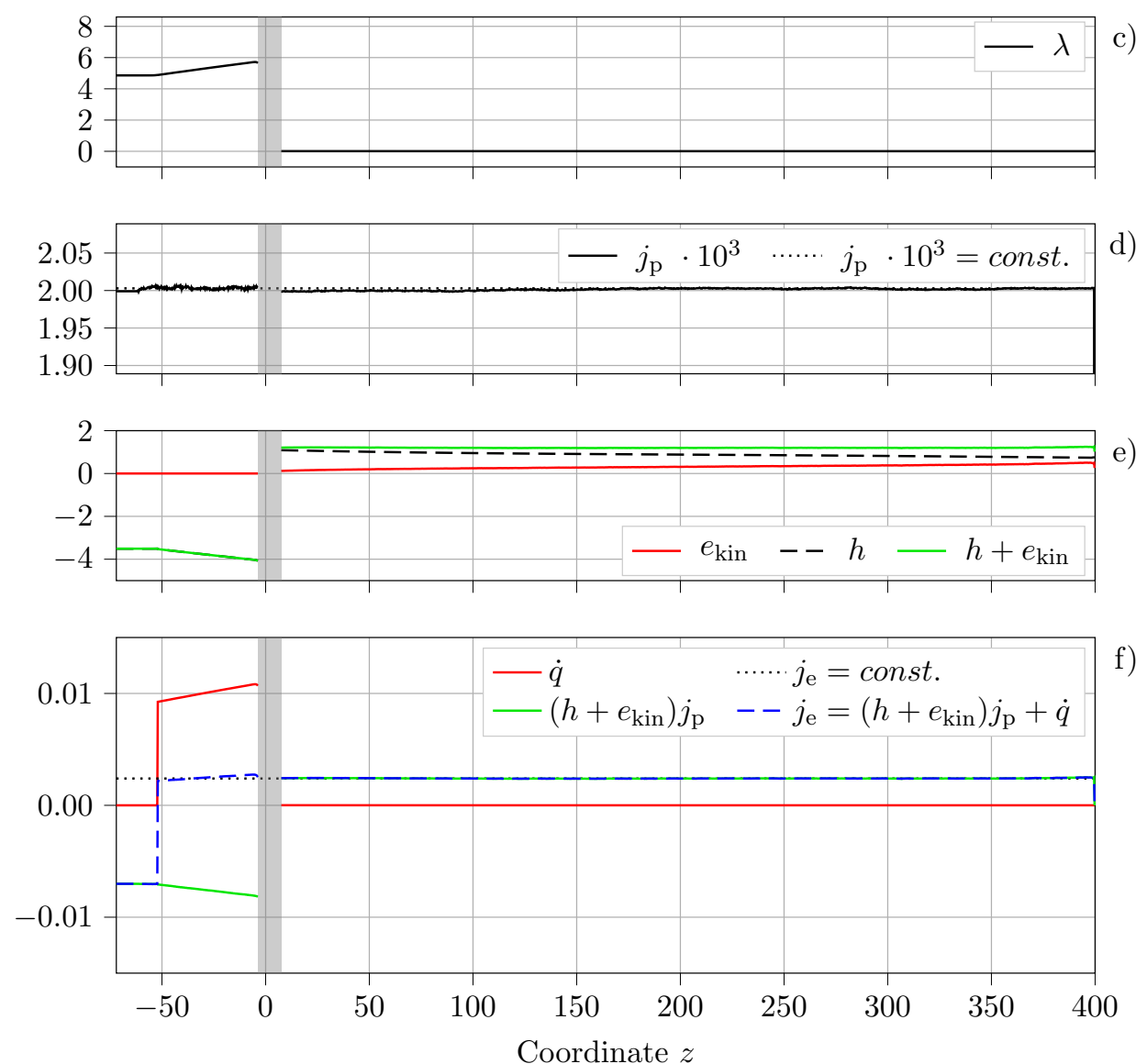

Figure 45. Evaluation of the energy flux $j_{\mathrm{e}}$ obtained by post-processing of profiles sampled during simulation with $T_{\text {liq }}=0.8$ and $L_{\mathrm{n}}=52$. Panels according to the description outlined above. 

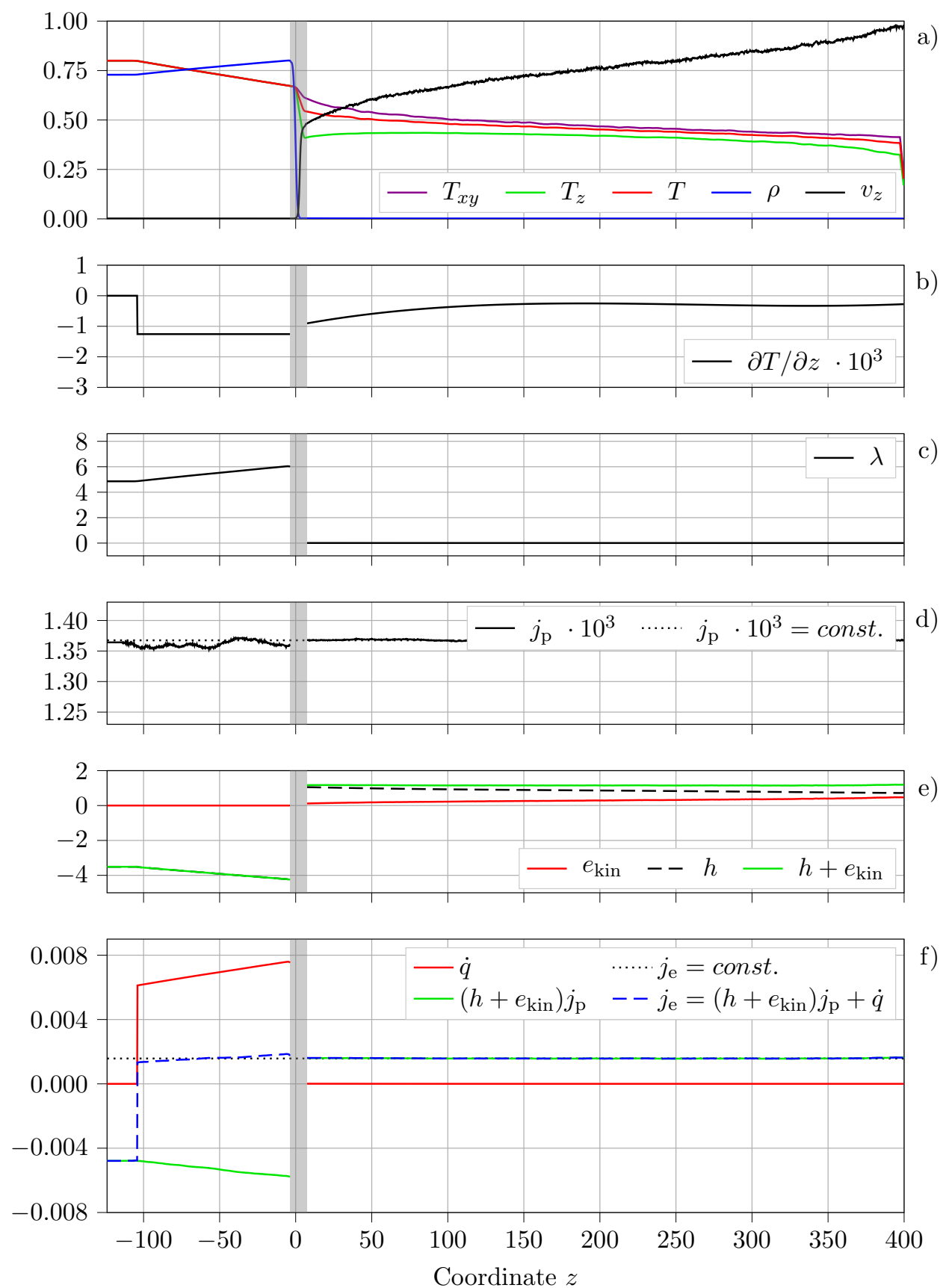

Figure 46. Evaluation of the energy flux $j_{\mathrm{e}}$ obtained by post-processing of profiles sampled during simulation with $T_{\text {liq }}=0.8$ and $L_{\mathrm{n}}=104$. Panels according to the description outlined above. 

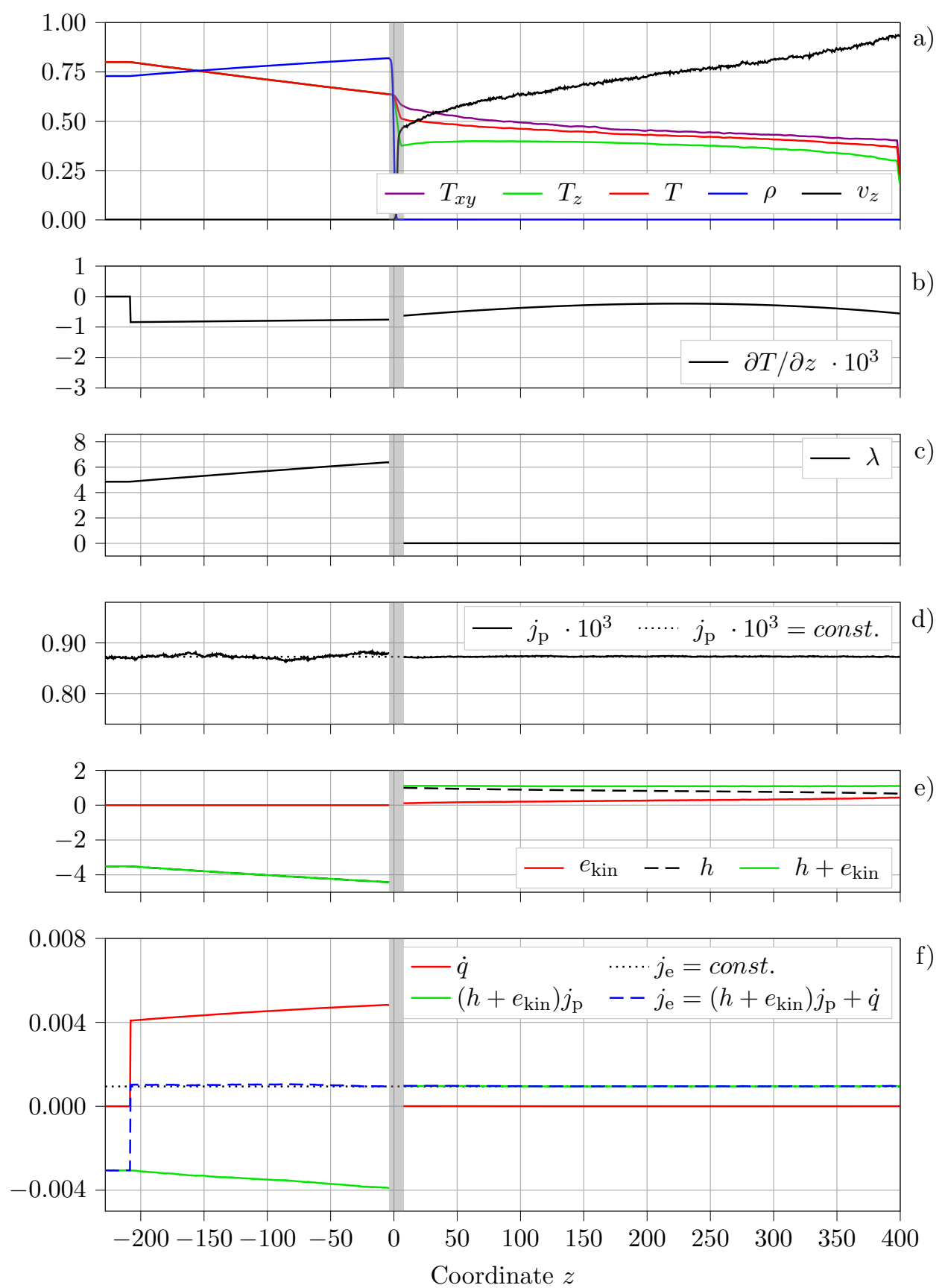

Figure 47. Evaluation of the energy flux $j_{\mathrm{e}}$ obtained by post-processing of profiles sampled during simulation with $T_{\text {liq }}=0.8$ and $L_{\mathrm{n}}=208$. Panels according to the description outlined above. 